\title{
Sections of fiber bundles over surfaces and TQFTs
}

\author{
Vladimir Turaev
}

\begin{abstract}
We study the existence problem and the enumeration problem for sections of Serre fibrations over compact orientable surfaces. When the fundamental group of the fiber is finite, a complete solution is given in terms of 2-dimensional cohomology classes associated with certain irreducible representations of this group. The proofs are based on Topological Quantum Field Theory.
\end{abstract}

Mathematics Subject Classification (2010). 57R20, 57R22.

Keywords. Surfaces, fibrations, sections, topological quantum field theory.

\section{Contents}

1 Introduction . . . . . . . . . . . . . . . . . . . . 275

2 Representations and cohomology classes . . . . . . . . . . . . . . . . . 279

3 Homotopy Quantum Field Theory ． . . . . . . . . . . . . . . . . . . . . . . . . . 282

4 Biangular $G$-algebras and 2-dimensional HQFTs . . . . . . . . . . . . . . . . . . 286

5 Reduction of Theorem 1.2 to a lemma . . . . . . . . . . . . . . . . . 293

6 Crossed $G$-algebras . . . . . . . . . . . . . . . . . . . . . . . . . . . . . 295

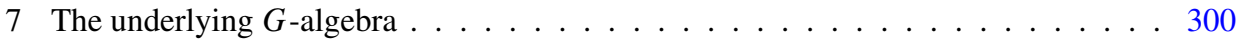

8 Properties of the underlying crossed $G$-algebras $\ldots \ldots \ldots$. . . . . . . . . 306

9 The $G$-center of a biangular $G$-algebra $\ldots \ldots \ldots$. . . . . . . . . . . . . 309

10 Proof of Lemma $5.2 \ldots \ldots \ldots 15$

11 The case of a trivial bundle . . . . . . . . . . . . . . . . . 317

References . . . . . . . . . . . . . . . . . . . 318

\section{Introduction}

A section of a fibration $p: E \rightarrow W$ is a continuous mapping $s: W \rightarrow E$ such that $p s=\mathrm{id}_{W}$. The existence problem for sections is fundamental in the theory of fibrations. If $p$ has sections, then one may ask about the number of their homotopy classes. We address these problems for Serre fibrations over compact orientable surfaces and solve them under certain assumptions on the fiber. 
Assume that $p: E \rightarrow W$ is a Serre fibration over a compact connected oriented surface $W$ of positive genus. If $\partial W \neq \emptyset$, then we fix a continuous section $s_{\partial}: \partial W \rightarrow$ $E$ of $p$ over $\partial W$ (so that $p s_{\partial}=\mathrm{id}_{\partial W}$ ) and search for its extensions to $W$. By definition, a section $s: W \rightarrow E$ of $p$ extends $s_{\partial}$ if $\left.s\right|_{\partial W}=s_{\partial}$. For closed $W$, this condition is void. The reader wishing to avoid technicalities related to $\partial W$ and $s_{\partial}$ may pursue the case $\partial W=\emptyset$.

One can approach the existence problem for sections via obstruction theory. To this end, fix base points $w \in$ Int $W=W-\partial W$ and $e \in p^{-1}(w) \subset E$. Set $\pi^{\prime}=\pi_{1}(E, e), \pi=\pi_{1}(W, w)$, and consider the homomorphism $p_{\#}: \pi^{\prime} \rightarrow \pi$ induced by $p$. A section of $p$ carrying $w$ to $e$ induces a right inverse $\pi \rightarrow \pi^{\prime}$ of

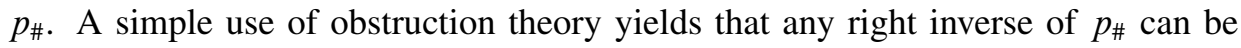
realized by a section $s$ of $p$. The condition $\left.s\right|_{\partial W}=s_{\partial}$ may also be translated into the algebraic language. However, this reformulation in terms of the fundamental groups is as difficult as the original problem and does not shed much light.

We give a necessary and sufficient condition for the existence of a section of $p$ extending $s_{\partial}$ in the case where the fundamental group of the fiber $F=p^{-1}(w)$ of $p$ is finite. Our condition involves 2-dimensional characteristic classes of $p: E \rightarrow W$ derived from certain linear representations of $\pi_{1}(F)$. If, additionally, $\pi_{2}(F)=0$, then we give a formula for the number of homotopy classes of such sections.

We now state our main theorems. Let $C_{1}, \ldots, C_{m}$ be the components of $\partial W$ endowed with orientation induced by that of $W$. Here $m \geq 0$ is the number of components of $\partial W$. For $k=1, \ldots, m$, fix an embedded path $c_{k}:[0,1] \rightarrow W$ leading from $w \in$ Int $W$ to a point of $C_{k}$. We assume that these $m$ paths meet only at $w$. For each $k=1, \ldots, m$, fix a lift $\tilde{c}_{k}:[0,1] \rightarrow E$ of $c_{k}$ leading from $e$ to $s_{\partial}\left(c_{k}(1)\right)$. Conjugating the loop $s_{\partial} \mid c_{k}$ by $\tilde{c}_{k}$, we obtain a loop in $E$ based at $e$. It represents a certain $\gamma_{k} \in \pi^{\prime}$.

Fix an algebraically closed field of characteristic zero $K$. Set $\Phi=\pi_{1}(F, e)$ and denote by $\operatorname{Irr}(\Phi)$ the set of equivalence classes of irreducible finite-dimensional linear representation of $\Phi$ over $K$. Since $\pi_{2}(W)=0$, the inclusion $F \hookrightarrow E$ induces an injection $\Phi \hookrightarrow \pi^{\prime}$ so that we can view $\Phi$ as a subgroup of $\pi^{\prime}$. Clearly, $\Phi=\operatorname{Ker}\left(p_{\#}: \pi^{\prime} \rightarrow \pi\right)$ is normal in $\pi^{\prime}$. The action of $\pi^{\prime}$ on $\Phi$ by conjugations induces an action of $\pi^{\prime}$ on $\operatorname{Irr}(\Phi)$. The restriction of the latter action to $\Phi$ is trivial, and we obtain an action of $\pi=\pi^{\prime} / \Phi$ on $\operatorname{Irr}(\Phi)$. Denote the fixed point set of this action by $I_{0}$. For any $\rho \in I_{0}$ and any $a \in \pi^{\prime}$, there is a matrix $M_{a} \in \operatorname{GL}_{\operatorname{dim} \rho}(K)$ such that $\rho\left(a^{-1} h a\right)=M_{a}^{-1} \rho(h) M_{a}$ for all $h \in \Phi$. Set $t_{\rho}(a)=0$ if $\operatorname{Tr} M_{a}=0$ and $t_{\rho}(a)=1$ if $\operatorname{Tr} M_{a} \neq 0$, where $\operatorname{Tr}$ is the matrix trace. The number $t_{\rho}(a)$ is welldefined because $M_{a}$ is unique up to multiplication by elements of $K^{*}=K \backslash\{0\}$. In Section 2 we associate with $\rho \in I_{0}$ and the set $\gamma=\left\{\gamma_{k}\right\}_{k=1}^{m} \subset \pi^{\prime}$ a cohomology class $\zeta^{\rho, \gamma} \in H^{2}\left(W, \partial W ; K^{*}\right)$.

Theorem 1.1. Let the fiber $F$ of $p: E \rightarrow W$ be path-connected and the group $\Phi=\pi_{1}(F, e)$ be finite. The section $s_{\partial}$ of $p$ over $\partial W$ extends to a section of $p$ over $W$ 
if and only if

$$
\sum_{\rho \in I_{0}}\left\{(\operatorname{dim} \rho)^{\chi(W)} \zeta^{\rho, \gamma}([W, \partial W]) \prod_{k=1}^{m} t_{\rho}\left(\gamma_{k}\right)\right\} \neq 0
$$

Here $\chi(W)$ is the Euler characteristic of $W$ and $\zeta^{\rho, \gamma}([W, \partial W]) \in K^{*}$ is the evaluation of $\zeta^{\rho, \gamma}$ on the fundamental class $[W, \partial W] \in H_{2}(W, \partial W ; \mathbb{Z})$. The evaluation is induced by the bilinear form $K^{*} \times \mathbb{Z} \rightarrow K^{*},(k, n) \mapsto k^{n}$.

For closed $W$, Theorem 1.1 says that $p$ has a section if and only if (1) holds. Note that for closed $W$, we have $\gamma=\emptyset, m=0$, and the product $\prod_{k=1}^{m} t_{\rho}\left(\gamma_{k}\right)=1$ may be deleted from (1).

The sum in (1) is finite because the sets $\operatorname{Irr}(\Phi)$ and $I_{0} \subset \operatorname{Irr}(\Phi)$ are finite. This sum is non-empty because the trivial 1-dimensional representation of $\Phi$ belongs to $I_{0}$. The individual terms in (1) may depend on the paths $c_{1}, \ldots, c_{m}$ but, as we shall see, their sum does not depend on these paths and is a non-negative rational number. Theorem 1.1 shows that $p$ has no sections extending $s_{\partial}$ if and only if this number takes its minimal possible value (equal to zero).

Theorem 1.1 follows from a Lefschetz-type formula for the number of extensions of $s_{\partial}$ to $W$ considered up to an appropriate equivalence relation. Here it is convenient to switch to pointed sections. Recall the base points $e \in E$ and $w=p(e) \in \operatorname{Int} W$. A section $s: W \rightarrow E$ of $p$ is pointed if $s(w)=e$. Two pointed sections of $p$ are homotopic if they can be deformed into each other relative to $\partial W$ in the class of pointed sections of $p$. Two pointed sections of $p$ are related by bubbling if they are equal outside a small open 2-disk $D \subset$ Int $W-\{w\}$. The restrictions of such two sections on the closed 2-disk $\bar{D} \subset W$ form a mapping $S^{2} \rightarrow E$, a "bubble". Two pointed sections of $p$ are bubble equivalent if they can be obtained from each other by a finite sequence of bubblings. The set of bubble equivalence classes of pointed sections of $p$ extending $s_{\partial}$ is denoted $S\left(p, s_{\partial}\right)$. Decomposing a deformation of a section into local deformations, one observes that homotopic pointed sections are bubble equivalent. If $\pi_{2}(F)=0$, then the converse is also true, and the bubble equivalence is just the homotopy.

Theorem 1.2. Let the fiber $F$ of $p: E \rightarrow W$ be path-connected and the group $\Phi=\pi_{1}(F, e)$ be finite. Then

$$
\left|S\left(p, s_{\partial}\right)\right|=|\Phi| \sum_{\rho \in I_{0}}\left\{(|\Phi| / \operatorname{dim} \rho)^{-\chi(W)} \zeta^{\rho, \gamma}([W, \partial W]) \prod_{k=1}^{m} t_{\rho}\left(\gamma_{k}\right)\right\},
$$

where the vertical bars stand for the cardinality of a set.

Theorem 1.2 implies Theorem 1.1 (one should observe that when $F$ is pathconnected, the bundle $p$ has a section if and only if $p$ has a pointed section). Theorems 1.1 and 1.2 are satisfactory from a topologist's viewpoint because they provide a complete solution to natural geometric problems concerning the bundle $p$ in terms 
of certain characteristic classes associated with $p$. Theorems 1.1 and 1.2 are new and non-trivial already in the case $\partial W=\emptyset$. They were announced in this case in [13].

The connectedness assumption in Theorems 1.1 and 1.2 is not a serious restriction. For a section of $p$ to exist, the homomorphism $p_{\#}: \pi^{\prime} \rightarrow \pi$ has to be surjective and so we must have $\pi_{0}(F)=\pi_{0}(E)$. It would be very interesting to find a version of these theorems for infinite $\Phi$.

The cohomology classes $\zeta^{\rho, \gamma}$ in (2) may be efficiently computed. This yields a number of interesting applications in topology and group theory. I mention here a few applications. In the case where $\partial W=\emptyset$ and $\Phi$ is finite abelian, the bundle $p: E \rightarrow W$ has a section if and only if the induced homomorphism $p_{*}: H_{2}(E ; \mathbb{Z}) \rightarrow$ $H_{2}(W ; \mathbb{Z})$ is surjective. The same equivalence holds for any finite $\Phi$ provided $\partial W=$ $\varnothing$ and the genus of $W$ is greater than or equal to $(1 / 2) \log _{2}|\Phi|$. For a deduction of these claims from Theorem 1.2, see [13]. Similar claims hold for $\partial W \neq \varnothing$, though the surjectivity of $p_{*}$ should be replaced with the condition that the 1-cycle $s_{\partial}(\partial W)$ is homologically trivial in $E$. Another application [13] concerns non-abelian cohomology of surfaces.

As a typical group-theoretic application of Theorem 1.2, I mention the following result of M. Natapov and myself [10]. Let $a, b$ be two commuting elements of a group $G$. Suppose that $G$ contains as a normal subgroup the quaternion group $Q=\{ \pm 1, \pm i, \pm j, \pm k\}$. Question: for how many pairs $\alpha, \beta \in Q$ the elements $a \alpha, b \beta \in G$ commute? Answer: the number of such pairs is equal to $8,16,24$, or 40 , and all the numbers $8,16,24,40$ are realized by some $G, a, b$. Similar results may be obtained for any finite group through a study of its representations.

Theorem 1.1 is uninteresting for trivial bundles (product fibrations) because they certainly have sections. Theorem 1.2 for trivial bundles is equivalent to the classical formula for the number of homomorphisms $\pi=\pi_{1}(W, w) \rightarrow \Phi$ due to Frobenius [6] and Mednykh [9]; see Section 11.

Theorem 1.2 can be extended to non-orientable surfaces. In another direction, the sections of $p: E \rightarrow W$ can be counted with weights determined by an element of $H^{2}\left(E, s_{\partial}(\partial W) ; K^{*}\right)$. This will be discussed elsewhere.

The proof of Theorem 1.2 is based on techniques of quantum topology and specifically utilizes 2-dimensional Homotopy Quantum Field Theory (HQFT). Note that HQFTs are versions of familiar Topological Quantum Field Theories (TQFTs) for manifolds and cobordisms endowed with maps into a certain space. HQFTs were introduced in my unpublished preprint [12]. Two-dimensional HQFTs with simply connected target were independently introduced in [2]. The bulk of the paper is devoted to a study of 2-dimensional HQFTs whose target is an Eilenberg-MacLane space of type $K(G, 1)$, where $G$ is a group. This study is strictly limited here to notions and results needed for the proof of Theorem 1.2. Our exposition is self-contained and does not require preliminary knowledge of TQFTs or HQFTs.

Here is an outline of the proof of Theorem 1.2. We rewrite the number $\left|S\left(p, s_{\partial}\right)\right|$ in terms of a 2-dimensional HQFT associated with $p_{\#}: \pi^{\prime} \rightarrow \pi$. Analyzing the underlying algebra of this HQFT, we split it as a direct sum of HQFTs numerated by 
elements of $\operatorname{Irr}(\Phi)$. Using the technique of transfer, we show that only the elements of $I_{0} \subset \operatorname{Irr}(\Phi)$ may contribute non-zero terms to $\left|S\left(p, s_{\partial}\right)\right|$. These terms are the summands in (2).

The Frobenius-Mednykh formula for the number of homomorphisms from the group $\pi_{1}(W)$ to a finite group was rediscovered in the context of TQFTs by Dijkgraaf and Witten [4] and by Freed and Quinn [5]. The present paper extends their work. It is plausible that Theorem 1.2 admits a purely algebraic proof generalizing the original methods of Frobenius and Mednykh. The author plans to study an algebraic approach to Theorem 1.2 elsewhere.

Throughout the paper, the symbol $K$ denotes an algebraically closed field of characteristic zero. The symbols $G$ and $G^{\prime}$ denote (discrete) groups.

My work on this paper was partially supported by the NSF grants DMS-0707078 and DMS-0904262.

\section{Representations and cohomology classes}

We define here (in a general algebraic setting) the cohomology classes $\zeta^{\rho, \gamma}$.

Throughout this section we fix a group epimorphism $q: G^{\prime} \rightarrow G$ with kernel $\Gamma$.

2.1. Representations of $\Gamma$. By a representation of $\Gamma$, we mean a group homomorphism $\rho: \Gamma \rightarrow \mathrm{GL}_{n}(K)$ with $n \geq 1$. Two representations $\rho: \Gamma \rightarrow \mathrm{GL}_{n}(K)$ and $\rho^{\prime}: \Gamma \rightarrow \mathrm{GL}_{n^{\prime}}(K)$ of $\Gamma$ are equivalent if $n=n^{\prime}$ and there is $M \in \mathrm{GL}_{n}(K)$ such that $\rho^{\prime}(h)=M^{-1} \rho(h) M$ for all $h \in \Gamma$. A representation $\rho: \Gamma \rightarrow \mathrm{GL}_{n}(K)$ is irreducible if the induced action of $\Gamma$ on $K^{n}$ preserves no linear subspace of $K^{n}$ except 0 and $K^{n}$. Let $\operatorname{Irr}(\Gamma)$ be the set of equivalence classes of irreducible representations of $\Gamma$. Given $a \in G^{\prime}$ and an irreducible representation $\rho: \Gamma \rightarrow \mathrm{GL}_{n}(K)$, the formula $h \mapsto \rho\left(a^{-1} h a\right): \Gamma \rightarrow \mathrm{GL}_{n}(K)$ defines an irreducible representation of $\Gamma$ denoted $a \rho$. This defines a left action of $G^{\prime}$ on $\operatorname{Irr}(\Gamma)$. The action of $\Gamma \subset G^{\prime}$ is trivial because $a \rho=\rho(a)^{-1} \rho \rho(a)$ for any $a \in \Gamma$. We obtain thus a left action of $G=G^{\prime} / \Gamma$ on $\operatorname{Irr}(\Gamma)$. The fixed point set of this action is denoted $I_{0}(q)$.

2.2. The cohomology class $\zeta^{\rho}$. For $\rho \in I_{0}(q)$, we define a cohomology class $\zeta^{\rho} \in H^{2}\left(G ; K^{*}\right)$. Set $n=\operatorname{dim} \rho \geq 1$. For each $\alpha \in G$, choose $\tilde{\alpha} \in q^{-1}(\alpha)$ so that $\tilde{1}=1$. Since $\rho \in I_{0}(q)$, there is a matrix $M_{\alpha} \in \mathrm{GL}_{n}(K)$ such that $\tilde{\alpha} \rho=M_{\alpha}^{-1} \rho M_{\alpha}$. By the Schur lemma, $M_{\alpha}$ is unique up to multiplication by elements of $K^{*}$. We call $M_{\alpha}$ the conjugating matrix (corresponding to $\tilde{\alpha}$ ) and fix it for all $\alpha \neq 1$. For $\alpha=1$, set $M_{\alpha}=E_{n}$. For $\alpha, \beta \in G$, set $L_{\alpha, \beta}=(\widetilde{\alpha \beta})^{-1} \tilde{\alpha} \tilde{\beta} \in \Gamma$.

Lemma 2.1. For any $\alpha, \beta \in G$, there is a unique $\zeta_{\alpha, \beta} \in K^{*}$ such that

$$
\zeta_{\alpha, \beta} M_{\alpha} M_{\beta}=M_{\alpha \beta} \rho\left(L_{\alpha, \beta}\right) .
$$


The family $\left\{\zeta_{\alpha, \beta}\right\}_{\alpha, \beta}$ is a normalized 2-cocycle on $G$. Its cohomology class $\zeta^{\rho} \in$ $H^{2}\left(G ; K^{*}\right)$ depends only on the equivalence class of $\rho$ and does not depend on the choice of the matrices $\left\{M_{\alpha}\right\}_{\alpha}$ and the lifts $\{\tilde{\alpha}\}_{\alpha}$.

Proof. For $\alpha, \beta \in G$ and $h \in \Gamma$,

$$
\begin{aligned}
M_{\alpha \beta}^{-1} \rho(h) M_{\alpha \beta}=(\widetilde{\alpha \beta} \rho)(h) & =\rho\left(\widetilde{\alpha \beta}^{-1} h \widetilde{\alpha \beta}\right) \\
& =\rho\left(L_{\alpha, \beta} \tilde{\beta}^{-1} \tilde{\alpha}^{-1} h \tilde{\alpha} \tilde{\beta} L_{\alpha, \beta}^{-1}\right) \\
& =\rho\left(L_{\alpha, \beta}\right) M_{\beta}^{-1} M_{\alpha}^{-1} \rho(h) M_{\alpha} M_{\beta} \rho\left(L_{\alpha, \beta}\right)^{-1}
\end{aligned}
$$

Since $\rho$ is irreducible, there is a unique $\zeta_{\alpha, \beta} \in K^{*}$ satisfying (3).

Any element of $G^{\prime}$ expands uniquely as $\tilde{\alpha} g$ with $\alpha \in G$ and $g \in \Gamma$. The formula $\bar{\rho}(\tilde{\alpha} g)=M_{\alpha} \rho(g)$ defines a mapping $\bar{\rho}: G^{\prime} \rightarrow \operatorname{GL}_{n}(K)$. Clearly, $\left.\bar{\rho}\right|_{\Gamma}=\rho$ and $\bar{\rho}(\tilde{\alpha})=M_{\alpha}$ for all $\alpha \in G$. Also, for all $a \in G^{\prime}$ and $h \in \Gamma$,

$$
\bar{\rho}(a) \rho(h)=\bar{\rho}(a h) .
$$

To see this, expand $a=\tilde{\alpha} g$ with $\alpha \in G$ and $g \in \Gamma$. We have

$$
\bar{\rho}(\tilde{\alpha} g) \rho(h)=M_{\alpha} \rho(g) \rho(h)=M_{\alpha} \rho(g h)=\bar{\rho}(\tilde{\alpha} g h) \text {. }
$$

Formula (4) implies that for any $\alpha, \beta \in G$,

$$
\bar{\rho}(\tilde{\alpha} \tilde{\beta})=\bar{\rho}(\widetilde{\alpha \beta}) \rho\left(L_{\alpha, \beta}\right)=M_{\alpha \beta} \rho\left(L_{\alpha, \beta}\right)=\zeta_{\alpha, \beta} M_{\alpha} M_{\beta}=\zeta_{\alpha, \beta} \bar{\rho}(\tilde{\alpha}) \bar{\rho}(\tilde{\beta}) .
$$

We claim that more generally, for all $a, b \in G^{\prime}$,

$$
\bar{\rho}(a b)=\zeta_{q(a), q(b)} \bar{\rho}(a) \bar{\rho}(b) .
$$

To see this, expand $a=\tilde{\alpha} g, b=\tilde{\beta} h$ with $\alpha=q(a), \beta=q(b) \in G$ and $g, h \in \Gamma$. Using (4) and (5), we obtain

$$
\begin{aligned}
\bar{\rho}(a b) & =\bar{\rho}\left(\tilde{\alpha} \tilde{\beta} \tilde{\beta}^{-1} g \tilde{\beta} h\right) \\
& =\bar{\rho}(\tilde{\alpha} \tilde{\beta}) \rho\left(\tilde{\beta}^{-1} g \tilde{\beta}\right) \rho(h) \\
& =\zeta_{\alpha, \beta} M_{\alpha} M_{\beta}(\tilde{\beta} \rho)(g) \rho(h) \\
& =\zeta_{\alpha, \beta} M_{\alpha} \rho(g) M_{\beta} \rho(h) \\
& =\zeta_{\alpha, \beta} \bar{\rho}(a) \bar{\rho}(b) .
\end{aligned}
$$

Formula (6) implies that $\left\{\zeta_{\alpha, \beta}\right\}_{\alpha, \beta}$ is a 2-cocycle on $G$. It is normalized in the sense that $\zeta_{1,1}=1$. That the cohomology class $\zeta^{\rho}$ of this cocyle does not depend on the choice of $\left\{M_{\alpha}\right\}_{\alpha}$ follows directly from (3). To prove the independence of $\zeta^{\rho}$ of the lifts $\{\tilde{\alpha}\}_{\alpha}$, suppose that each $\tilde{\alpha}$ is traded for $\tilde{\alpha}^{\prime}=\tilde{\alpha} g_{\alpha}$ with $g_{\alpha} \in \Gamma$. Then $M_{\alpha}$ is traded for $M_{\alpha}^{\prime}=M_{\alpha} \rho\left(g_{\alpha}\right)$. For $\alpha, \beta \in G$,

$$
L_{\alpha, \beta}^{\prime}=\left(\widetilde{\alpha \beta}^{\prime}\right)^{-1} \tilde{\alpha}^{\prime} \tilde{\beta}^{\prime}=g_{\alpha \beta}^{-1} \widetilde{\alpha \beta}^{-1} \tilde{\alpha} g_{\alpha} \tilde{\beta} g_{\beta}=g_{\alpha \beta}^{-1} L_{\alpha, \beta}\left(\tilde{\beta}^{-1} g_{\alpha} \tilde{\beta}\right) g_{\beta}
$$


Substituting these new values of $M, L$ in (3) and using the equality $\rho\left(\tilde{\beta}^{-1} g_{\alpha} \tilde{\beta}\right)=$ $M_{\beta}^{-1} \rho\left(g_{\alpha}\right) M_{\beta}$, we obtain that the lifts $\tilde{\alpha}^{\prime}$ lead to the same cocycle $\left\{\zeta_{\alpha, \beta}\right\}$. When $\rho$ is conjugated by $M \in \mathrm{GL}_{n}(K)$, the matrices $\left\{M_{\alpha}\right\}_{\alpha}$ are replaced by $\left\{M^{-1} M_{\alpha} M\right\}_{\alpha}$ and the cocycle $\left\{\zeta_{\alpha, \beta}\right\}_{\alpha, \beta}$ is also preserved.

2.3. The function $t_{\rho}$ and the cohomology class $\zeta^{\rho, \gamma}$. A representation $\rho \in I_{0}(q)$ determines a function $t_{\rho}: G^{\prime} \rightarrow\{0,1\}$ as follows. For any $a \in G^{\prime}$, we have $a \rho=M^{-1} \rho M$ with $M \in \mathrm{GL}_{n}(K)$, where $n=\operatorname{dim} \rho$. Since $M$ is unique up to multiplication by elements of $K^{*}$, so is $\operatorname{Tr} M \in K$. We say that $a$ is $\rho$-adequate if $\operatorname{Tr} M \neq 0$. Let $t_{\rho}: G^{\prime} \rightarrow\{0,1\}$ be the function sending all $\rho$-adequate elements of $G^{\prime}$ to 1 and all other elements of $G^{\prime}$ to 0 . The set of $\rho$-adequate elements of $G^{\prime}$ and the function $t_{\rho}$ depend only on the equivalence class of $\rho$. A subset of $G^{\prime}$ is $\rho$-adequate if all its elements are $\rho$-adequate.

We say that a set $\gamma \subset G^{\prime}$ is $q$-free if $\gamma$ freely generates a (free) subgroup $\langle\gamma\rangle$ of $G^{\prime}$ and the restriction of $q$ to $\langle\gamma\rangle$ is injective. We derive from a $q$-free set $\gamma$ and any $\rho \in I_{0}(q)$ a cohomology class $\zeta^{\rho, \gamma} \in H^{2}\left(G, H ; K^{*}\right)$, where $H=q(\langle\gamma\rangle)=$ $\langle q(\gamma)\rangle \subset G$. If $\gamma$ is not $\rho$-adequate, set $\zeta^{\rho, \gamma}=0$. Suppose that $\gamma$ is $\rho$-adequate. Then for any $a \in \gamma$, there is a unique matrix $v(a) \in \mathrm{GL}_{n}(K)$ such that $a \rho=$ $v(a)^{-1} \rho v(a)$ and $\operatorname{Tr} v(a)=1$. The mapping $\gamma \rightarrow \mathrm{GL}_{n}(K), a \mapsto v(a)$ induces a group homomorphism $\langle\gamma\rangle \rightarrow \mathrm{GL}_{n}(K)$ denoted $v$. We define a normalized 2-cocycle $\left\{\zeta_{\alpha, \beta}\right\}_{\alpha, \beta}$ on $G$ as in Section 2.2 using the following choices: for all $\alpha \in H$, let $\tilde{\alpha}$ be the only element of $q^{-1}(\alpha) \cap\langle\gamma\rangle$ and $M_{\alpha}=v(\tilde{\alpha})$ (the lifts of elements of $G-H$ to $G^{\prime}$ and the associated matrices $M$ are chosen in an arbitrary way). It follows from the definitions that $\zeta_{\alpha, \beta}=1$ for all $\alpha, \beta \in H$. The proof of Lemma 2.1 shows that the cocycle $\left\{\zeta_{\alpha, \beta}\right\}_{\alpha, \beta}$ is well defined up to coboundaries of 1-cochains $G \rightarrow K^{*}$ sending $H$ to 1 . The cohomology class $\zeta^{\rho, \gamma} \in H^{2}\left(G, H ; K^{*}\right)$ of this cocycle depends only on the equivalence class of $\rho$. The natural homomorphism $H^{2}\left(G, H ; K^{*}\right) \rightarrow$ $H^{2}\left(G ; K^{*}\right)$ carries $\zeta^{\rho, \gamma}$ to $\zeta^{\rho}$ if $\gamma$ is $\rho$-adequate and to 0 otherwise. For example, the empty subset of $G^{\prime}$ is $q$-free and $\zeta^{\rho, \emptyset}=\zeta^{\rho} \in H^{2}\left(G,\{1\} ; K^{*}\right)=H^{2}\left(G ; K^{*}\right)$.

2.4. Theorems 1.1 and 1.2 re-examined. We can now explain all notation in Theorems 1.1 and 1.2. Applying Section 2.1 to

$$
G^{\prime}=\pi^{\prime}, \quad G=\pi, \quad q=p_{\#}: \pi^{\prime} \rightarrow \pi, \quad \Gamma=\Phi,
$$

we obtain an action of $\pi$ on $\operatorname{Irr}(\Phi)$. Every $\rho$ in the fixed point set of this action $I_{0}=$ $I_{0}\left(p_{\#}\right)$ yields a function $t_{\rho}: \pi^{\prime} \rightarrow\{0,1\}$ by Section 2.3. The set $\gamma=\left\{\gamma_{k}\right\}_{k=1}^{m} \subset \pi^{\prime}$ defined before the statement of Theorem 1.1 is $q$-free because $p_{\#}(\gamma)$ generates a free subgroup of $\pi$ of rank $m=|\gamma|$. Here it is essential that the genus of $W$ is positive. Therefore each $\rho \in I_{0}$ determines $\zeta^{\rho, \gamma} \in H^{2}\left(\pi,\langle q(\gamma)\rangle ; K^{*}\right)=H^{2}\left(W, \partial W ; K^{*}\right)$. This completes the statement of Theorems 1.1 and 1.2. 


\section{Homotopy Quantum Field Theory}

Throughout this section we fix an integer $d \geq 0$ and a connected CW-space $X$ with base point $x$.

3.1. Preliminaries. By a manifold, we mean an oriented topological manifold. A manifold $M$ is pointed if every component of $M$ is provided with a base point. The set of base points of $M$ is denoted $M_{\bullet}$.

A $d$-dimensional $X$-manifold is a pair (a pointed closed $d$-dimensional manifold $M$, a map $g: M \rightarrow X$ such that $g\left(M_{\bullet}\right)=x$ ). A disjoint union of $X$-manifolds is an $X$-manifold in the obvious way. An $X$-homeomorphism of $X$-manifolds $(M, g) \rightarrow$ $\left(M^{\prime}, g^{\prime}\right)$ is an orientation-preserving homeomorphism $f: M \rightarrow M^{\prime}$ such that $g=$ $g^{\prime} f$ and $g\left(M_{\bullet}\right)=M_{\bullet}^{\prime}$. An empty set is viewed as a pointed manifold and an $X$ manifold of any given dimension.

By a $(d+1)$-dimensional cobordism, we mean a triple $\left(W, M_{0}, M_{1}\right)$ where $W$ is a compact $(d+1)$-dimensional manifold and $M_{0}, M_{1}$ are disjoint pointed closed $d$-dimensional submanifolds of $\partial W$ such that $\partial W=\left(-M_{0}\right) \amalg M_{1}$. The manifold $W$ is not supposed to be pointed. As usual, $-M$ is $M$ with reversed orientation.

An $X$-cobordism is a cobordism $\left(W, M_{0}, M_{1}\right)$ endowed with a map $g: W \rightarrow X$ carrying the base points of $M_{0}, M_{1}$ to $x$. Both bases $M_{0}$ and $M_{1}$ of $W$ are considered as $X$-manifolds with maps to $X$ obtained by restricting $g$. An $X$-homeomorphism of $X$-cobordisms

$$
F:\left(W, M_{0}, M_{1}, g\right) \rightarrow\left(W^{\prime}, M_{0}^{\prime}, M_{1}^{\prime}, g^{\prime}\right)
$$

is an orientation-preserving and base point preserving homeomorphism of triples $\left(W, M_{0}, M_{1}\right) \rightarrow\left(W^{\prime}, M_{0}^{\prime}, M_{1}^{\prime}\right)$ such that $g=g^{\prime} F$. The standard operations on cobordisms (disjoint union, gluing along bases, etc.) apply in this setting in the obvious way. For brevity, we shall often omit the maps from the notation for $X$-manifolds and $X$-cobordisms.

3.2. Axioms of HQFTs. We adapt Atiyah's axioms of a TQFT to the present setting. A $(d+1)$-dimensional Homotopy Quantum Field Theory with target $X$ or, shorter, a $(d+1)$-dimensional $X$-HQFT assigns a finite-dimensional vector space $A_{M}$ over $K$ to any $d$-dimensional $X$-manifold $M$, an isomorphism $f_{\#}: A_{M} \rightarrow A_{M^{\prime}}$ to any $X$-homeomorphism of $d$-dimensional $X$-manifolds $f: M \rightarrow M^{\prime}$, and a homomorphism $\tau(W): A_{M_{0}} \rightarrow A_{M_{1}}$ to any $(d+1)$-dimensional $X$-cobordism $\left(W, M_{0}, M_{1}\right)$. The following seven axioms should be met.

(1) For any $X$-homeomorphisms of $d$-dimensional $X$-manifolds $f: M \rightarrow M^{\prime}$, $f^{\prime}: M^{\prime} \rightarrow M^{\prime \prime}$, we have $\left(f^{\prime} f\right)_{\#}=f_{\#}^{\prime} f_{\text {\# }}$. 
(2) For any $X$-homeomorphism (8), the following diagram is commutative:

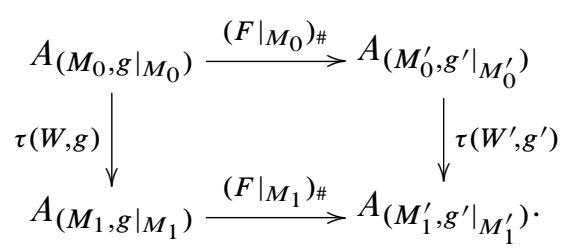

(3) If an $X$-cobordism $\left(W, M_{0}, M_{1}\right)$ is obtained from two $(d+1)$-dimensional $X$-cobordisms $\left(W_{0}, M_{0}, N\right)$ and $\left(W_{1}, N^{\prime}, M_{1}\right)$ by gluing along an $X$-homeomorphism $f: N \rightarrow N^{\prime}$, then

$$
\tau(W)=\tau\left(W_{1}\right) \circ f_{\#} \circ \tau\left(W_{0}\right): A_{M_{0}} \rightarrow A_{M_{1}} .
$$

(4) $A_{\emptyset}=K$, and for any disjoint $d$-dimensional $X$-manifolds $M, N$, there is a natural isomorphism $A_{M \amalg N}=A_{M} \otimes A_{N}$ (for a detailed formulation of naturality, see [11], p. 121). Here and below $\otimes=\otimes_{K}$.

(5) If a $(d+1)$-dimensional $X$-cobordism $W$ is a disjoint union of $X$-cobordisms $W_{1}, W_{2}$, then $\tau(W)=\tau\left(W_{1}\right) \otimes \tau\left(W_{2}\right)$.

(6) For any $d$-dimensional $X$-manifold $(M, g: M \rightarrow X)$, $\tau(M \times[0,1], M \times\{0\}, M \times\{1\}, g \circ \mathrm{pr}: M \times[0,1] \rightarrow X)=\mathrm{id}: A_{M} \rightarrow A_{M}$, where pr: $M \times[0,1] \rightarrow M$ is the projection. Here we identify $A_{M \times\{t\}}=A_{M}$ for $t=0,1$ via the canonical homeomorphism $M \times\{t\} \approx M$.

(7) For any $(d+1)$-dimensional $X$-cobordism $(W, g: W \rightarrow X)$, the homomorphism $\tau(W)$ is preserved under homotopies of $g$ constant on $\partial W$.

If $X=\{x\}$, then all references to maps to $X$ are redundant, and $X$-HQFTs are the familiar TQFTs. Note a few properties of HQFTs generalizing the standard properties of TQFTs, see [11]. Given a $(d+1)$-dimensional $X$-HQFT $(A, \tau)$, we associate with any $d$-dimensional $X$-manifold $M$ the bilinear form

$$
\eta_{M}=\tau(M \times[0,1], M \times\{0\} \cup(-M) \times\{1\}, \emptyset): A_{M} \otimes A_{-M} \rightarrow K .
$$

This form is non-degenerate, natural with respect to $X$-homeomorphisms, multiplicative with respect to disjoint union, and symmetric in the sense that $\eta_{-M}=\eta_{M} \circ \sigma$ where $\sigma$ is the standard flip $A_{-M} \otimes A_{M} \rightarrow A_{M} \otimes A_{-M}$. By definition, $-\emptyset=\emptyset$ and $\eta_{\varnothing}: K \otimes K \rightarrow K$ is the multiplication in $K$.

For a compact $(d+1)$-dimensional manifold $W$ with pointed boundary and a map $\left(W,(\partial W)_{\bullet}\right) \rightarrow(X, x)$, the $\operatorname{HQFT}(A, \tau)$ yields the vector space $A_{\partial W}$ and two homomorphisms $\tau(W, \emptyset, \partial W): K=A_{\emptyset} \rightarrow A_{\partial W}$ and $\tau(-W, \partial W, \emptyset): A_{\partial W} \rightarrow A_{\emptyset}=K$. Let $\tau(W) \in A_{\partial W}$ be the image of the unity $1_{K}$ under the first homomorphism. The second homomorphism is nothing but the image of $\tau(-W)$ under the isomorphism $A_{-\partial W} \approx \operatorname{Hom}_{K}\left(A_{\partial W}, K\right)$ induced by $\eta_{\partial W}$. The vector $\tau(W)$ is invariant under 
homotopy of the given map $W \rightarrow X$, natural with respect to $X$-homeomorphisms, and multiplicative under disjoint union.

Two $(d+1)$-dimensional $X$-HQFTs $(A, \tau)$ and $\left(A^{\prime}, \tau^{\prime}\right)$ are isomorphic if there is a family of $K$-isomorphisms $\left\{\rho_{M}: A_{M} \rightarrow A_{M}^{\prime}\right\}_{M}$, where $M$ runs over all $d$-dimensional $X$-manifolds, such that: $\rho_{\emptyset}=\operatorname{id}_{K}$ and $\rho_{M U N}=\rho_{M} \otimes \rho_{N}$ for any disjoint $X$-manifolds $M, N$; the natural square diagrams associated with $X$-homeomorphisms and $X$-cobordisms are commutative.

3.3. Example. Each $(d+1)$-dimensional $K^{*}$-valued singular cocycle $\theta$ on $X$ gives rise to a $(d+1)$-dimensional $X$-HQFT $(A, \tau)$. Let $M=(M, g: M \rightarrow X)$ be a $d$-dimensional $X$-manifold. Then $A_{M}$ is a one-dimensional $K$-vector space defined as follows. A $d$-dimensional singular cycle $a \in C_{d}(M ; \mathbb{Z})$ is a fundamental cycle of $M$ if it represents the sum of the fundamental homology classes of the components of $M$. Every such $a$ determines a generating vector $\langle a\rangle \in A_{M}=K\langle a\rangle$. If $a, b$ are two fundamental cycles of $M$, then $a-b=\partial c$ for $c \in C_{d+1}(M ; \mathbb{Z})$. We impose the equality $\langle b\rangle=g^{*}(\theta)(c)\langle a\rangle$, where $g^{*}(\theta)$ is the singular cocycle on $M$ obtained by pushing back $\theta$ along $g$. It is easy to check that $g^{*}(\theta)(c)$ does not depend on the choice of $c$ and that $A_{M}$ is a well-defined one-dimensional vector space. If $M=\emptyset$, then $a=0$ and by definition $A_{M}=K$ and $\langle a\rangle=1_{K} \in K$. An $X$-homeomorphism of $X$-manifolds $f: M \rightarrow M^{\prime}$ induces an isomorphism $f_{\#}: A_{M} \rightarrow A_{M^{\prime}}$ by $f_{\#}(\langle a\rangle)=$ $\left\langle f_{*}(a)\right\rangle$ for any fundamental cycle $a$ of $M$.

Given a $(d+1)$-dimensional $X$-cobordism $\left(W, M_{0}, M_{1}, g: W \rightarrow X\right)$, pick a singular chain $B \in C_{d+1}(W ; \mathbb{Z})$ such that $\partial B=b_{1}-b_{0}$, where $b_{0}, b_{1}$ are fundamental cycles of $M_{0}, M_{1}$, respectively. We also require $B$ to be fundamental in the sense that its image in $C_{d+1}(W, \partial W ; \mathbb{Z})$ is a fundamental cycle of $W$. We define $\tau(W): A_{M_{0}} \rightarrow A_{M_{1}}$ by $\tau(W)\left(\left\langle b_{0}\right\rangle\right)=g^{*}(\theta)(B)\left\langle b_{1}\right\rangle$. It is an exercise in singular homology to show that $\tau(W)$ is well defined and the axioms of an HQFT are met, cf. [12]. This HQFT is denoted $\left(A^{\theta}, \tau^{\theta}\right)$. Its isomorphism class depends only on the cohomology class of $\theta$.

For $\partial W=\emptyset$, we have $\tau(W, g)=g^{*}(\theta)([W])=\theta\left(g_{*}([W])\right)$. We can view $\left(A^{\theta}, \tau^{\theta}\right)$ as a device extending to cobordisms the evaluation of $\theta$ on cycles in $X$. Another such device (in the smooth category) is provided by the Cheeger-Simons differential characters. For a comparison, see [14].

3.4. Operations on HQFTs. We need three operations on HQFTs: direct sum, rescaling, and transfer. The direct sum of $(d+1)$-dimensional $X$-HQFTs $\left(A^{1}, \tau^{1}\right)$, $\left(A^{2}, \tau^{2}\right)$ is defined as follows. Set $\left(A^{1} \oplus A^{2}\right)_{M}=A_{M}^{1} \oplus A_{M}^{2}$ for any connected $d$-dimensional $X$-manifold $M$ and extend this to non-connected $M$ via Axiom (4) above. The action of $X$-homeomorphisms is defined by applying $\oplus$ and $\otimes$ to the actions provided by $\left(A^{1}, \tau^{1}\right),\left(A^{2}, \tau^{2}\right)$. For a $d$-dimensional $X$-manifold $M$ and $k=1,2$, we have a natural embedding $i_{M}^{k}: A_{M}^{k} \rightarrow\left(A^{1} \oplus A^{2}\right)_{M}$ and a natural projection $p_{M}^{k}:\left(A^{1} \oplus A^{2}\right)_{M} \rightarrow A_{M}^{k}$ such that $p_{M}^{k} i_{M}^{k}=$ id. For a connected 
$(d+1)$-dimensional $X$-cobordism $(W, M, N)$, set

$$
\left(\tau^{1} \oplus \tau^{2}\right)(W)=i_{N}^{1} \tau^{1}(W) p_{M}^{1}+i_{N}^{2} \tau^{2}(W) p_{M}^{2}:\left(A^{1} \oplus A^{2}\right)_{M} \rightarrow\left(A^{1} \oplus A^{2}\right)_{N} .
$$

This extends to non-connected $X$-cobordisms via Axiom (5) and gives a $(d+1)$ dimensional $X$-HQFT $\left(A^{1} \oplus A^{2}, \tau^{1} \oplus \tau^{2}\right)$.

One way to rescale a $(d+1)$-dimensional $X$-HQFT consists in multiplying the homomorphism associated with each $X$-cobordism $(W, M, N)$ by $k^{\chi(W)-\chi(M)}$ with fixed $k \in K^{*}$. For $d=1$, we shall use a slightly subtler version of this transformation. Given $k \in K^{*}$, the $k$-rescaling transforms a 2-dimensional $X$-HQFT into the same HQFT except that the homomorphism associated with each $X$-cobordism $(W, M, N)$ is multiplied by $k^{\left(\chi(W)+b_{0}(M)-b_{0}(N)\right) / 2}$. Here $b_{0}(M)=\operatorname{dim} H_{0}(M ; \mathbb{R})$ and we use the inclusion $\chi(W)+b_{0}(M)-b_{0}(N) \in 2 \mathbb{Z}$.

The transfer of HQFTs is defined in the following setting. Let $p: \tilde{X} \rightarrow X$ be a finite-sheeted (unramified) covering. Consider the path-connected pointed space $Y=\tilde{X} / p^{-1}(x)$ and let $q: \tilde{X} \rightarrow Y$ be the projection. The transfer derives from a $(d+1)$-dimensional HQFT $(A, \tau)$ with target $Y$ a $(d+1)$-dimensional HQFT $(\tilde{A}, \tilde{\tau})$ with $\operatorname{target} X$ as follows. For a $d$-dimensional $X$-manifold $(M, g: M \rightarrow X)$, consider all lifts of $g$ to $\tilde{X}$, i.e., all maps $\tilde{g}: M \rightarrow \widetilde{X}$ such that $p \tilde{g}=g$. The set of such $\tilde{g}$ is finite (possibly, empty). Each pair $(M, q \tilde{g})$ is an $Y$-manifold. Set

$$
\tilde{A}_{M}=\underset{\tilde{g}, p \tilde{g}=g}{\bigoplus} A_{(M, q \tilde{g})}
$$

Given an $X$-homeomorphism of $X$-manifolds $f:(M, g) \rightarrow\left(M^{\prime}, g^{\prime}\right)$, a lift $\tilde{g}: M \rightarrow$ $\tilde{X}$ of $g$ induces a lift $\tilde{g} f^{-1}: M^{\prime} \rightarrow \widetilde{X}$ of $g^{\prime}$. The HQFT $(A, \tau)$ provides an isomorphism $f_{\#}: A_{(M, q \tilde{g})} \rightarrow A_{\left(M^{\prime}, q \tilde{g} f^{-1}\right)}$. The direct sum of the latter over all $\tilde{g}$ is the isomorphism $f_{\#}: \tilde{A}_{(M, g)} \rightarrow \tilde{A}_{\left(M^{\prime}, g^{\prime}\right)}$ determined by $(\tilde{A}, \tilde{\tau})$.

Given a $(d+1)$-dimensional $X$-cobordism $\left(W, M_{0}, M_{1}, g: W \rightarrow X\right)$, there is a finite set of maps $\tilde{g}: W \rightarrow \tilde{X}$ such that $p \tilde{g}=g$. Each such $\tilde{g}$ restricted to $M_{0}, M_{1}$ yields certain lifts, $\tilde{g}_{0}, \tilde{g}_{1}$, of the maps $g_{0}=\left.g\right|_{M_{0}}: M_{0} \rightarrow X$ and $g_{1}=\left.g\right|_{M_{1}}: M_{1} \rightarrow X$, respectively. We define $\tilde{\tau}(W, g): \tilde{A}_{M_{0}} \rightarrow \tilde{A}_{M_{1}}$ to be the sum over all $\tilde{g}$ of the homomorphisms $\tau(W, q \tilde{g}): A_{\left(M_{0}, q \tilde{g}_{0}\right)} \rightarrow A_{\left(M_{1}, q \tilde{g}_{1}\right)}$. Then $(\tilde{A}, \tilde{\tau})$ is a $(d+1)$-dimensional $X$-HQFT.

For example, for $d \geq 1$, any $\theta \in H^{d+1}\left(\tilde{X} ; K^{*}\right)=H^{d+1}\left(Y ; K^{*}\right)$ determines a $Y$-HQFT $\left(A^{\theta}, \tau^{\theta}\right)$. Its transfer $\left(\tilde{A}^{\theta}, \tilde{\tau}^{\theta}\right)$ is an $X$-HQFT. For a $d$-dimensional $X$-manifold $(M, g: M \rightarrow X)$, the dimension of $\tilde{A}_{M}^{\theta}$ is equal to the number of lifts of $g$ to $\tilde{X}$. For a closed $(d+1)$-dimensional $X$-manifold $(W, g: W \rightarrow X)$, we have $\tilde{\tau}^{\theta}(W, g)=\sum_{\tilde{g}} \tilde{g}^{*}(\theta)([W])$, where $\tilde{g}$ runs over all lifts of $g$ to $\tilde{X}$.

3.5. Aspherical targets. $\mathrm{A}(d+1)$-dimensional $\operatorname{HQFT}(A, \tau)$ with aspherical target $X$ may be reformulated in terms of homotopy classes of maps to $X$ rather than maps themselves. By homotopy we mean homotopy in the class of maps sending the base points to $x \in X$. Here is the key observation: for any pointed closed 
$d$-dimensional manifold $M$ and for any maps $g_{0}, g_{1}: M \rightarrow X$ related by a homotopy $H:\left(M \times[0,1], M_{\bullet} \times[0,1]\right) \rightarrow(X, x)$, the pair $(M \times[0,1], H)$ is an $X$-cobordism, and the associated homomorphism $\tau(M \times[0,1], H): A_{M, g_{0}} \rightarrow A_{M, g_{1}}$ does not depend on the choice of $H$. This follows from Axiom (7) of an HQFT since any two such $H$ are homotopic, which is a consequence of the asphericity of $X$. Composing $H$ with the inverse homotopy and using Axiom (6), we obtain that $\tau(M \times[0,1], H)$ is an isomorphism. We identify the vector spaces $A_{M, g}$ (with $g: M \rightarrow X$ in the given homotopy class) along these isomorphisms. The resulting vector space depends only on the homotopy class and has the same dimension as $A_{M, g}$. The action of $X$-homeomorphisms and of $X$-cobordisms is compatible with these identifications. As a consequence, dealing with HQFTs with aspherical target $X$, we can safely replace maps of manifolds and cobordisms to $X$ by pointed homotopy classes of maps.

From now on, we will consider only $X$-HQFTs with aspherical $X$ and will modify the notions of $X$-manifolds and $X$-cobordisms accordingly (i.e., view the maps to $X$ up to pointed homotopy). By $X$-homeomorphisms we will mean orientationpreserving and base point-preserving homeomorphisms commuting with the given homotopy classes of maps to $X$.

Lemma 3.1. If $f: M \rightarrow N$ is an $X$-homeomorphism of d-dimensional $X$-manifolds and if a homeomorphism $f^{\prime}:\left(M, M_{\bullet}\right) \rightarrow\left(N, N_{\bullet}\right)$ is isotopic to $f$ in the class of homeomorphisms $\left(M, M_{\bullet}\right) \rightarrow\left(N, N_{\bullet}\right)$, then $f^{\prime}$ is an $X$-homeomorphism and $f_{\#}^{\prime}=f_{\#}: A_{M} \rightarrow A_{N}$ for any $(d+1)$-dimensional $X-\operatorname{HQFT}(A, \tau)$.

The first claim of this lemma follows from the definitions. The second claim follows from Axioms (2) and (6) of an HQFT.

An aspherical space $X$ with base point $x$ is an Eilenberg-MacLane space of type $K(G, 1)$ for $G=\pi_{1}(X, x)$. By Section 3.3, every $\theta \in H^{d+1}\left(G ; K^{*}\right)=$ $H^{d+1}\left(X ; K^{*}\right)$ gives rise to a $(d+1)$-dimensional $X$-HQFT $\left(A^{\theta}, \tau^{\theta}\right)$. More generally, consider a subgroup $H \subset G$ of finite index and the associated covering

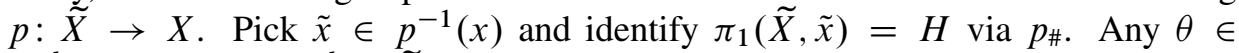
$H^{d+1}\left(H ; K^{*}\right)=H^{d+1}\left(\widetilde{X} ; K^{*}\right)$ with $d \geq 1$ yields a $(d+1)$-dimensional $X$-HQFT through transfer. For $d=1$ and $k \in K^{*}$, we can $k$-rescale this HQFT. The resulting $X$-HQFT is denoted $\left(A^{G, H, \theta}, \tau^{G, H, \theta, k}\right)$. It can also be obtained by first $k$-rescaling the HQFT $\left(A^{\theta}, \tau^{\theta}\right)$ and then transferring to $X$.

\section{Biangular $G$-algebras and 2-dimensional HQFTs}

For a group $G$, we introduce a class of $G$-graded algebras giving rise to 2-dimensional HQFTs with target $X=K(G, 1)$. This generalizes the construction of 2-dimensional TQFTs $(G=1)$ from associative algebras in [1] and [7]. 
4.1. Biangular $G$-algebras. A $G$-graded algebra or, briefly, a $G$-algebra is an associative algebra $B$ over $K$ endowed with a splitting $B=\bigoplus_{\alpha \in G} B_{\alpha}$ such that all $B_{\alpha}$ are finite-dimensional, $B_{\alpha} B_{\beta} \subset B_{\alpha \beta}$ for any $\alpha, \beta \in G$, and $B$ has a (right and left) unit $1_{B} \in B_{1}$ where 1 is the neutral element of $G$. An inner product on a $G$-algebra $B$ is a symmetric $K$-bilinear form $\eta: B \otimes B \rightarrow K$ such that

(1) for all $\alpha \in G$, the restriction of $\eta$ on $B_{\alpha} \otimes B_{\alpha^{-1}}$ is non-degenerate,

(2) $\eta\left(B_{\alpha} \otimes B_{\beta}\right)=0$ if $\alpha \beta \neq 1$,

(3) $\eta(a, b)=\eta\left(a b, 1_{B}\right)$ for any $a, b \in B$.

For $c \in B$, the symbol $\mu_{c}$ denotes the homomorphism $B \rightarrow B, x \mapsto c x$. A $G$-algebra $B=\bigoplus_{\alpha \in G} B_{\alpha}$ is biangular if it has an inner product $\eta: B \otimes B \rightarrow K$ such that for any $\alpha, \beta \in G$ and $a \in B_{\alpha}, b \in B_{\alpha^{-1}}$,

$$
\eta(a, b)=\operatorname{Tr}\left(\left.\mu_{a b}\right|_{B_{\beta}}: B_{\beta} \rightarrow B_{\beta}\right) .
$$

The form $\eta$ (if it exists) is unique and denoted $\eta_{B}$. The conditions above imply that for any $c \in B_{1}$, the trace of $\mu_{c}: B_{\beta} \rightarrow B_{\beta}$ does not depend on $\beta$ and is equal to $\eta_{B}\left(c, 1_{B}\right)=\eta_{B}\left(1_{B}, c\right)$. In particular, $\operatorname{dim}\left(B_{\beta}\right)=\operatorname{dim}\left(B_{1}\right)=\eta_{B}\left(1_{B}, 1_{B}\right)$ for all $\beta \in G$. Note that conditions (2) and (3) for $\eta_{B}$ and the symmetry of $\eta_{B}$ may be deduced from (9).

Given a biangular $G$-algebra $B$ and $\alpha \in G$, we can view the vector spaces $B_{\alpha}$ and $B_{\alpha^{-1}}$ as dual to each other via $\eta_{B}$. Pick a basis $\left\{p_{i}^{\alpha}\right\}_{i}$ of $B_{\alpha}$, consider the dual basis $\left\{q_{i}^{\alpha}\right\}_{i}$ of $B_{\alpha^{-1}}$, and set $b_{\alpha}=\sum_{i} p_{i}^{\alpha} \otimes q_{i}^{\alpha} \in B_{\alpha} \otimes B_{\alpha^{-1}}$. The vector $b_{\alpha}$ does not depend on the choice of $\left\{p_{i}^{\alpha}\right\}_{i}$. The system of vectors $\left\{b_{\alpha}\right\}_{\alpha}$ is symmetric in the sense that $b_{\alpha^{-1}}$ is obtained from $b_{\alpha}$ by permutation of the tensor factors for all $\alpha \in G$. Note that for all $\alpha \in G$,

$$
\sum_{i} p_{i}^{\alpha} q_{i}^{\alpha}=1_{B}
$$

This follows from the non-degeneracy of $\eta_{B}$ and the fact that for any $c \in B_{1}$,

$$
\eta\left(c, \sum_{i} p_{i}^{\alpha} q_{i}^{\alpha}\right)=\sum_{i} \eta\left(c p_{i}^{\alpha}, q_{i}^{\alpha}\right)=\operatorname{Tr}\left(\left.\mu_{c}\right|_{B_{\alpha}}: B_{\alpha} \rightarrow B_{\alpha}\right)=\eta_{B}\left(c, 1_{B}\right) .
$$

Also, for any $a \in B_{\alpha^{-1}}$ and $b \in B_{\alpha}$,

$$
\sum_{i} \eta\left(a, p_{i}^{\alpha}\right) \eta\left(b, q_{i}^{\alpha}\right)=\eta\left(a, \sum_{i} \eta\left(b, q_{i}^{\alpha}\right) p_{i}^{\alpha}\right)=\eta(a, b) .
$$

For example, if $q: G^{\prime} \rightarrow G$ is a group epimorphism with finite kernel $\Gamma$, then the group algebra $B=K\left[G^{\prime}\right]$ is a biangular $G$-algebra, where $B_{\alpha}=K\left[q^{-1}(\alpha)\right] \subset B$ for all $\alpha \in G$. Observe that $\eta_{B}(a, b)=|\Gamma|$ if $a, b \in G^{\prime}$ satisfy $a b=1$ and $\eta_{B}(a, b)=0$ for all other $a, b \in G^{\prime}$. For $\alpha \in G$, we have $b_{\alpha}=|\Gamma|^{-1} \sum_{a \in q^{-1}(\alpha)} a \otimes a^{-1}$. More general examples of biangular $G$-algebras may be derived from the twisted group algebras of $G^{\prime}$ associated with $K^{*}$-valued 2-cocycles on $G^{\prime}$. 
4.2. $G$-systems. Recall the standard combinatorial description of maps from a $\mathrm{CW}$ complex $T$ to $X=K(G, 1)$. By vertices, edges, and faces of $T$, we mean 0 -cells, 1-cells, and 2-cells of $T$, respectively. Denote the set of vertices of $T$ by $\operatorname{Vert}(T)$ and the set of oriented edges of $T$ by $\operatorname{Edg}(T)$. Each $e \in \operatorname{Edg}(T)$ leads from an initial vertex $i_{e}$ to a terminal vertex $t_{e}$ (they may coincide). The orientation reversal defines a free involution $e \mapsto e^{-1}$ on $\operatorname{Edg}(T)$.

A face $\Delta$ of $T$ is adjoined to the 1-skeleton $T^{(1)}$ of $T$ along a (continuous) map $f_{\Delta}: S^{1} \rightarrow T^{(1)}$. We call $T$ regular if for any face $\Delta$ of $T$, the set $f_{\Delta}^{-1}(\operatorname{Vert}(T)) \subset S^{1}$ is a finite non-empty set which splits $S^{1}$ into arcs mapped by $f_{\Delta}$ homeomorphically onto edges of $T$. These arcs in $S^{1}$ are called the sides of $\Delta$. The image $f_{\Delta}(e)$ of a side $e$ of $\Delta$ is an edge of $T$ called the underlying edge of $e$. We shall often make no difference between sides and their underlying edges. An orientation of $\Delta$ induces an orientation and a cyclic order $e_{1}, e_{2}, \ldots, e_{n}$ on the set of sides of $\Delta$. Here $n \geq 1$ is the number of sides and $t_{e_{r}}=i_{e_{r+1}}$ for all $r(\bmod n)$. The corresponding cyclically ordered oriented edges of $T$ form the boundary of $\Delta$.

A $G$-system on a regular CW-complex $T$ is a mapping $\operatorname{Edg}(T) \rightarrow G, e \mapsto g_{e}$ such that

(1) $g_{e^{-1}}=\left(g_{e}\right)^{-1}$ for any $e \in \operatorname{Edg}(T)$,

(2) if ordered oriented edges $e_{1}, e_{2}, \ldots, e_{n}$ of $T$ with $n \geq 1$ form the boundary of a face of $T$, then $g_{e_{1}} g_{e_{2}} \ldots g_{e_{n}}=1$.

For any set $V \subset \operatorname{Vert}(T)$, we define an equivalence relation, called $V$-homotopy, on the set of $G$-systems on $T$. Two $G$-systems $g, g^{\prime}$ on $T$ are $V$-homotopic if there is a map $v: \operatorname{Vert}(T) \rightarrow G$ such that $v(V)=1$ and $g_{e}^{\prime}=v\left(i_{e}\right) g_{e}\left(v\left(t_{e}\right)\right)^{-1}$ for all $e \in \operatorname{Edg}(T)$. For $V=\emptyset$, we use the term homotopy for $V$-homotopy.

A $G$-system $g$ on $T$ gives rise to a map $|g|:|T| \rightarrow X$, where $|T|$ is the underlying topological space of $T$. This map carries $\operatorname{Vert}(T)$ to the base point $x \in X$ and carries each $e \in \operatorname{Edg}(T)$ into a loop in $X$ representing $g_{e} \in G=\pi_{1}(X, x)$. The map $g \mapsto|g|$ induces a bijection between the $V$-homotopy classes of $G$-systems on $T$ and the homotopy classes of maps $(|T|, V) \rightarrow(X, x)$.

4.3. State sums on closed surfaces. Fix a biangular $G$-algebra $B=\bigoplus_{\alpha \in G} B_{\alpha}$. Let $W$ be a closed $X$-surface, i.e., a closed oriented surface endowed with a map $W \rightarrow X=K(G, 1)$. We define $\tau_{B}(W) \in K$ as follows.

Pick a regular CW-decomposition $T$ of $W$ (for example, a triangulation). By a flag of $T$, we mean a pair (a face $\Delta$ of $T$, a side $e$ of $\Delta$ ). The flag $(\Delta, e)$ induces an orientation on $e$ such that $\Delta$ lies on the right of $e$. This means that the pair (a vector looking from a point of $e$ into $\Delta$, the oriented edge of $T$ underlying $e$ ) is positive with respect to the given orientation of $W$.

Let $g$ be a $G$-system on $T$ representing the homotopy class of the given map $|T|=W \rightarrow X$ (here $V=\emptyset$ ). With each flag $(\Delta, e)$ of $T$ we associate the $K$-module $B(\Delta, e, g)=B_{g_{e}}$ where $e$ is oriented so that $\Delta$ lies on its right.

Every edge $e$ of $T$ appears in two flags, $\left(\Delta_{1}, e\right),\left(\Delta_{2}, e\right)$, and inherits from them 
opposite orientations. Since the corresponding values of $g$ are mutually inverse, Section 4.1 yields a vector $b_{g_{e}} \in B\left(\Delta_{1}, e, g\right) \otimes B\left(\Delta_{2}, e, g\right)$. Set

$$
b_{g}=\bigotimes_{e} b_{g_{e}} \in \underset{(\Delta, e)}{\bigotimes} B(\Delta, e, g)
$$

where on the left-hand side $e$ runs over all unoriented edges of $T$ and on the right-hand side $(\Delta, e)$ runs over all flags of $T$.

Let $\Delta$ be a face of $T$ with $n$ sides. We orient and cyclically order the sides $e_{1}, e_{2}, \ldots, e_{n}$ of $\Delta$ so that $\Delta$ lies on their right. Then $g_{e_{1}} g_{e_{2}} \ldots g_{e_{n}}=1$. The form

$$
B\left(\Delta, e_{1}, g\right) \otimes B\left(\Delta, e_{2}, g\right) \otimes \cdots \otimes B\left(\Delta, e_{n}, g\right) \rightarrow K,
$$

defined by

$$
a_{1} \otimes a_{2} \otimes \cdots \otimes a_{n} \mapsto \eta_{B}\left(a_{1} a_{2} \ldots a_{n}, 1_{B}\right)
$$

is invariant under cyclic permutations. The tensor product of these forms over all faces of $T$ is a homomorphism $d_{g}: \bigotimes_{(\Delta, e)} B(\Delta, e, g) \rightarrow K$. Set $\langle g\rangle=d_{g}\left(b_{g}\right) \in K$.

The following lemma is the key result underlying the state sum construction of a 2-dimensional HQFT from a biangular $G$-algebra.

Lemma 4.1. $\tau_{B}(W)=\langle g\rangle \in K$ depends only on the homotopy class of the given map $W \rightarrow X$.

Proof. We need to prove that $\langle g\rangle$ does not depend on the choice of $g$ in its homotopy class and does not depend on the choice of $T$. It is convenient to switch to the dual language of skeletons of $W$. A skeleton of $W$ is a finite graph on $W$ whose complementary regions are open 2-disks. A skeleton may have loop edges (i.e., edges with coinciding endpoints) and multiple edges (i.e., different edges with the same endpoints). A regular CW-decomposition $T$ of $W$ determines a skeleton $S_{T}$ of $W$. It is obtained by placing a "central" point in each face of $T$ and connecting the centers of any two faces adjacent to the same 1-cell $e$ of $T$ by an edge meeting $e$ transversely in one point and disjoint from the rest of $T^{(1)}$. This establishes an equivalence between regular $\mathrm{CW}$-decompositions and skeletons. The definitions of a $G$-system $g$ on $T$ and of $\langle g\rangle$ can be easily rewritten in terms of labelings of the oriented edges of skeletons.

We define two local moves on a skeleton $S$ of $W$. The contraction move contracts an edge of $S$ with distinct endpoints. The corresponding move on $\mathrm{CW}$ decompositions erases an open edge adjacent to two distinct faces. The loop move on $S$ removes a loop edge bounding a disk in $W-S$. The corresponding move on $\mathrm{CW}$-decompositions erases a vertex of valency 1 and the incident open edge. The contraction moves, the loop moves, and their inverses are called basic moves. Using the theory of spines of surfaces, one shows that any two skeletons of $W$ can be related by a finite sequence of basic moves and ambient isotopy in $W$. The invariance 
of $\langle g\rangle$ under the loop move and the contraction move follows from (10) and (11), respectively.

It remains to prove the invariance of $\langle g\rangle$ under an arbitrary homotopy move $g \mapsto g^{\prime}$. In terms of the dual skeleton $S$ of $W$, the move is determined by a certain map $v: \pi_{0}(W-S) \rightarrow G$. The transformation $g \mapsto g^{\prime}$ is given by the formula $g^{\prime}(f)=v(U) g(f)(v(V))^{-1}$, where $f$ is any oriented edge of $S$ and $U, V$ are the components of $W-S$ lying on the right and on the left of $f$, respectively (possibly, $U=V)$. An easy induction reduces the desired equality $\langle g\rangle=\left\langle g^{\prime}\right\rangle$ to the case where $v$ takes value $1 \in G$ on all components of $W-S$ except a single component $U$, where this value is $v_{0} \in G$. We say in this case that $g^{\prime}$ is obtained from $g$ by a $v_{0}$-move at $U$. We now expand the $v_{0}$-move $g \mapsto g^{\prime}$ as a composition of several basic moves. By the invariance of $\langle g\rangle$ under the basic moves, this will imply that $\langle g\rangle=\left\langle g^{\prime}\right\rangle$.

Pick a small embedded loop $e$ in $U$ based at a vertex $x$ of $S$ adjacent to $U$. The loop $e$ splits $U$ into a small disk bounded by $e$ and a complementary open 2-disk $D$. Then $S_{1}=S \cup e$ is a skeleton of $W$ obtained from $S$ by an inverse loop move, and $D$ a component of $W-S_{1}$. Let $g_{1}$ be the $G$-system on $S_{1}$ extending the $G$-system $g$ on $S$ by $g_{1}(e)=1 \in G$. Let $g_{1}^{\prime}$ be the $G$-system on $S_{1}$ obtained from $g_{1}$ by the $v_{0}$-move at $D$. Clearly, $\left.g_{1}^{\prime}\right|_{S}=g^{\prime}$. Now we modify the skeleton $S_{1}=S \cup e$ keeping $S$ and keeping one endpoint of $e$ at $x$ and sliding the second endpoint of $e$ along the consecutive sides of $U$. We do this until the second endpoint traverses the entire boundary of $U$ and comes back to $x$ from the other side. During this deformation, $e$ becomes an embedded arc except at the beginning and the end when $e$ is an embedded loop based at $x$. Note that when the moving endpoint of $e$ traverses a vertex of $S$ adjacent to $U$, the skeleton $S \cup e$ is transformed via a composition of a contraction move with an inverse contraction move. Under these moves, the $G$-systems $g_{1}, g_{1}^{\prime}$ on $S \cup e$ are transformed in a canonical way keeping the values on all edges except the contracted ones. Throughout the deformation, the $G$-systems $g_{1}, g_{1}^{\prime}$ remain related by the $v_{0}$-move at the image of $D$ under the deformation. At the end of the deformation, $S_{1}$ is transformed into a skeleton $S_{2}$ of $W$ isotopic to $S_{1}$ and $g_{1}, g_{1}^{\prime}$ are transformed into $G$-systems $g_{2}, g_{2}^{\prime}$ on $S_{2}$ related by the $v_{0}$-move at the component of $W-S_{2}$ obtained as the image of $D$ under the deformation. This component is a small 2-disk bounded by $e$. Applying the loop move, we transform $g_{2}, g_{2}^{\prime}$ into one and the same $G$-system $g_{3}$ on $S$. This gives six sequences of basic moves $g \mapsto g_{1} \mapsto g_{2} \mapsto g_{3} \mapsto g_{2}^{\prime} \mapsto g_{1}^{\prime} \mapsto g^{\prime}$ whose composition is the move $g \mapsto g^{\prime}$.

4.4. A pseudo-HQFT. The invariant $\tau_{B}$ of closed $X$-surfaces derived above from a biangular $G$-algebra $B$ is extended here to a 2-dimensional "pseudo-HQFT" ( $A=$ $\left.A_{B}, \tau=\tau_{B}\right)$ with target $X=K(G, 1)$. The prefix "pseudo" appears here for two reasons. First of all, the pseudo-HQFT $(A, \tau)$ applies to 1-dimensional $X$-manifolds with a certain additional structure called framing. Secondly, $(A, \tau)$ fails to satisfy Axiom (6) of Section 3.2. This pseudo-HQFT will be transformed into a genuine HQFT in the next subsection. 
By $X$-curves, we shall mean 1-dimensional $X$-manifolds. A framed $X$-curve is a triple $(M, T, g)$, where $M$ is a pointed closed 1-dimensional manifold, $T$ is a $\mathrm{CW}$-decomposition of $M$ such that $M . \subset \operatorname{Vert}(T)$ and $g$ is a $G$-system on $T$. This $G$-system gives rise to a homotopy class of maps $|g|:\left(M, M_{0}\right) \rightarrow(X, x)$, where $x$ is the base point of $X$. This makes $M$ into an $X$-curve and allows us to consider the framing $(T, g)$ as an additional structure on this $X$-curve.

We associate with a framed $X$-curve $M=(M, T, g)$ the finite-dimensional vector space $A_{M}=\bigotimes_{e} B_{g_{e}}$, where $e$ runs over the edges of $T$ endowed with the orientation induced by that of $M$. By definition, $M=\emptyset$ is framed and $A_{\emptyset}=K$. Note that $A_{M} \amalg N=A_{M} \otimes A_{N}$ for all $M, N$.

An $X$-homeomorphism of framed $X$-curves is a homeomorphism of the underlying manifolds preserving the base points, the orientation, the framing, and the $G$-system. Such homeomorphisms act on the associated vector spaces in the obvious tautological way.

Consider a 2-dimensional $X$-cobordism $\left(W, M_{0}, M_{1}\right)$ such that both $X$-curves $M_{0}$ and $M_{1}$ are framed. We define a $K$-homomorphism $\tau(W): A_{M_{0}} \rightarrow A_{M_{1}}$ as follows. Pick a regular CW-decomposition $T$ of $W$ extending the given $C W$-decompositions of $M_{0}$ and $M_{1}$. A cellular approximation for the given map $W \rightarrow X$ yields a $G$-system $g$ on $T$ such that $g$ extends the $G$-systems on $M_{0}$ and $M_{1}$ provided by the framing and the induced map $|g|:\left(W,(\partial W)_{\bullet}\right) \rightarrow(X, x)$ is in the given homotopy class. Flags $(\Delta, e)$ of $T$ and the vector spaces $B(\Delta, e, g)=B_{g_{e}}$ are defined as in Section 4.3. As in Section 4.3, we have a homomorphism $d_{g}: \bigotimes_{(\Delta, e)} B(\Delta, e, g) \rightarrow K$, where $(\Delta, e)$ runs over all flags of $T$, and a vector

$$
b_{g}=\bigotimes_{e} b_{g_{e}} \in \bigotimes_{(\Delta, e \subset \operatorname{Int} W)} B(\Delta, e, g)
$$

where $e$ runs over all edges of $T$ lying in Int $W$ and $(\Delta, e)$ runs over all flags of $T$ such that $e \subset$ Int $W$. Contracting $d_{g}$ with $b_{g}$, we obtain a homomorphism

$$
\langle g\rangle: \bigotimes_{e \subset M_{0} \cup M_{1}} B\left(\Delta_{e}, e, g\right) \rightarrow K .
$$

Since $\partial W=M_{1} \cup\left(-M_{0}\right)$, the face of $T$ adjacent to $e \subset M_{r}$ lies on the left of $e$ for $r=1$ and on the right of $e$ for $r=0$. Therefore

$$
B\left(\Delta_{e}, e, g\right)= \begin{cases}B_{g_{e}} & \text { if } e \subset M_{0}, \\ B_{g_{e}-1}=\left(B_{g_{e}}\right)^{*} & \text { if } e \subset M_{1}\end{cases}
$$

Here we identify $B_{g_{e} e^{-1}}$ with the dual of $B_{g_{e}}$ using the inner product $\eta_{B}$ on $B$. Thus, the homomorphism (13) is adjoint to a homomorphism

$$
A_{M_{0}}=\bigotimes_{e \subset M_{0}} B_{g_{e}} \rightarrow \bigotimes_{e \subset M_{1}} B_{g_{e}}=A_{M_{1}}
$$

denoted $\tau(W)$. 
Lemma 4.2. The homomorphism $\tau(W)$ does not depend on the choice of $T$ and $g$. (It depends only on the framings of the $X$-curves $M_{0}, M_{1}$ and the homotopy class of the given map $(W,(\partial W).) \rightarrow(X, x))$.

The proof goes along the same lines as the proof of Lemma 4.1. The only difference is that a skeleton of $W$ meets $\partial W$ along a set of 1-valent vertices that are the centers of the 1-cells of $M_{0} \cup M_{1}$.

Lemma 4.3. Let $M_{0}, M_{1}, N$ be three framed $X$-curves. If a 2-dimensional $X$ cobordism $\left(W, M_{0}, M_{1}\right)$ is obtained by gluing two $X$-cobordisms $\left(W_{0}, M_{0}, N\right)$ and $\left(W_{1}, N, M_{1}\right)$ along $N$, then $\tau(W)=\tau\left(W_{1}\right) \circ \tau\left(W_{0}\right): A_{M_{0}} \rightarrow A_{M_{1}}$.

Proof. For $r=0,1$, pick a regular CW-decomposition $T_{r}$ of $W_{r}$ extending the given $\mathrm{CW}$-decompositions of the bases. Let $g_{r}$ be a $G$-system on $T_{r}$ representing the given map $W_{r} \rightarrow X$. Gluing $T_{0}$ and $T_{1}$ along $N$ we obtain a regular CW-decomposition, $T$, of $W$. Let $g$ be the unique $G$-system on $T$ extending $g_{0}$ and $g_{1}$. Formula (11) implies that $\langle g\rangle=\left\langle g_{1}\right\rangle \circ\left\langle g_{0}\right\rangle: A_{M_{0}} \rightarrow A_{M_{1}}$.

To sum up, the rule $M \mapsto A_{M}, W \mapsto \tau(W)$ defines a sort of HQFT for framed $X$-curves and for $X$-cobordisms with framed bases. This "pseudo-HQFT" $(A, \tau)$ satisfies all axioms of Section 3.2 except possibly Axiom (6).

4.5. A 2-dimensional HQFT. We now derive from the pseudo-HQFT ( $A=A_{B}$, $\left.\tau=\tau_{B}\right)$ a genuine HQFT, cf. [Tu2], Section VII.3. For any $X$-curve $M$, denote by $\mathcal{T}_{M}$ the class of all framings $(T, g)$ of $M$. Observe that for any framings $t_{0}, t_{1} \in \mathcal{T}_{M}$, the cylinder $W=M \times[0,1]$ (mapped to $X$ via the composition of the projection $W \rightarrow M$ with the given map $M \rightarrow X)$ is an $X$-cobordism between the framed $X$-curves $\left(M, t_{0}\right)$ and $\left(M, t_{1}\right)$. Set

$$
p\left(t_{0}, t_{1}\right)=\tau(W): A_{\left(M, t_{0}\right)} \rightarrow A_{\left(M, t_{1}\right)} .
$$

By Lemma 4.3, $p\left(t_{0}, t_{2}\right)=p\left(t_{1}, t_{2}\right) p\left(t_{0}, t_{1}\right)$ for any $t_{0}, t_{1}, t_{2} \in \mathcal{T}_{M}$. In particular, $p\left(t_{0}, t_{0}\right)$ is a projector onto a subspace $A_{\left(M, t_{0}\right)}^{\circ}$, of $A_{\left(M, t_{0}\right)}$. Moreover, $p\left(t_{0}, t_{1}\right)$ maps $A_{\left(M, t_{0}\right)}^{\circ}$ isomorphically onto $A_{\left(M, t_{1}\right)}^{\circ}$. The vector spaces $\left\{A_{(M, t)}^{\circ}\right\}_{t \in \mathcal{T}_{M}}$ and the isomorphisms $\left\{p\left(t_{0}, t_{1}\right): A_{\left(M, t_{0}\right)}^{\circ} \rightarrow A_{\left(M, t_{1}\right)}^{\circ}\right\}_{t_{0}, t_{1} \in \mathcal{T}_{M}}$ form a projective system of vector spaces and isomorphisms. The corresponding projective limit is a vector space denoted $A_{M}^{\circ}$. By definition, for any framing $t$ of $M$, we have a canonical isomorphism $A_{M}^{\circ} \cong A_{(M, t)}^{\circ}$. In other words, we have a canonical embedding $A_{M}^{\circ} \subset A_{(M, t)}$ and a canonical projection $P_{M}: A_{(M, t)} \rightarrow A_{M}^{\circ}$ which is the identity on $A_{M}^{\circ}$.

To define the action $f_{\#}: A_{M}^{\circ} \rightarrow A_{M^{\prime}}^{\circ}$ of an $X$-homeomorphism $f: M \rightarrow M^{\prime}$, pick a framing $t$ of $M$ and consider the framing $t^{\prime}=f(t)$ of $M^{\prime}$. Then $f_{\#}$ is the composition of the identification isomorphism $A_{M}^{\circ} \cong A_{(M, t)}^{\circ}$ with the isomorphism $A_{(M, t)}^{\circ} \cong A_{\left(M^{\prime}, t^{\prime}\right)}^{\circ}$ induced by $f$ and with the identification isomorphism $A_{\left(M^{\prime}, t^{\prime}\right)}^{\circ} \cong$ $A_{M^{\prime}}^{\circ}$. This composition is independent of the choice of $t$. 
A 2-dimensional $X$-cobordism $\left(W, M_{0}, M_{1}\right)$ splits as a union of collars of $M_{0}$, $M_{1}$ with a copy of $W$. Therefore for any framings $t_{0}, t_{1}$ of $M_{0}, M_{1}$, we have

$$
\tau(W)=P_{M_{1}} \tau(W) P_{M_{0}}: A_{\left(M_{0}, t_{0}\right)} \rightarrow A_{\left(M_{1}, t_{1}\right)} .
$$

Let $\tau^{\circ}(W): A_{M_{0}}^{\circ} \rightarrow A_{M_{1}}^{\circ}$ be the restriction of $\tau(W)$ to $A_{M_{0}}^{\circ} \subset A_{\left(M_{0}, t_{0}\right)}$. This homomorphism does not depend on $t_{0}, t_{1}$ and enters the commutative diagram

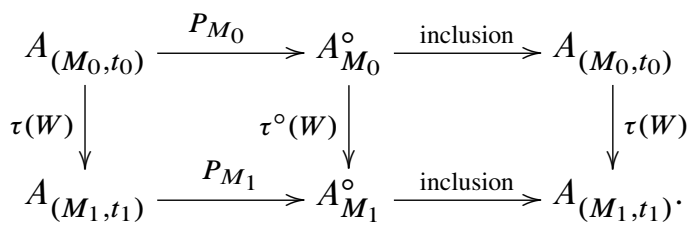

Theorem 4.4. The rule $M \mapsto A_{M}^{\circ}, W \mapsto \tau^{\circ}(W)$ defines an $X-H Q F T$.

This theorem follows from the results of Section 4.4. To stress the role of the biangular $G$-algebra, $B$, we shall write $A_{B}^{\circ}$ for $A^{\circ}$ and $\tau_{B}^{\circ}$ for $\tau^{\circ}$. If $W$ is a closed $X$-surface, then $\tau_{B}^{\circ}(W)=\tau_{B}(W)$.

The vector space $A_{M}^{\circ}$ associated with a connected $X$-curve $M$ may be described in terms of the $G$-algebra $B$ as follows. The $X$-curve $M$ yields a loop in $(X, x)$ representing an element $\alpha=\alpha(M)$ of $G=\pi_{1}(X, x)$. The $X$-curve $M$ admits a canonical framing $t$ having only one vertex (located at the base point of $M$ ) and one edge. Then $A_{(M, t)}=B_{\alpha}$ and $A_{M}^{\circ}$ is the image of the projector $P_{M}: B_{\alpha} \rightarrow B_{\alpha}$. We shall compute $P_{M}$ in Section 9. Note that each $\gamma \in B_{\alpha}$ yields a vector $[\gamma]=P_{M}(\gamma)$ in $A_{M}^{\circ}$.

\section{Reduction of Theorem 1.2 to a lemma}

Consider a group epimorphism $q: G^{\prime} \rightarrow G$ with finite kernel $\Gamma$. The biangular $G$-algebra $B=K\left[G^{\prime}\right]$ derived from $q$ in Section 4.1 determines a 2-dimensional HQFT $\left(A^{\circ}=A_{B}^{\circ}, \tau^{\circ}=\tau_{B}^{\circ}\right)$ with target $X=K(G, 1)$. We compute $\left(A^{\circ}, \tau^{\circ}\right)$ in two different ways and deduce Theorem 1.2.

5.1. Lemmas. Let $W$ be a compact connected oriented surface with $m \geq 0$ pointed boundary components $C_{1}, \ldots, C_{m}$ endowed with orientation induced by that of $W$. For $k=1, \ldots, m$, fix an embedded path $c_{k}:[0,1] \rightarrow W$ leading from a base point $w \in \operatorname{Int} W$ to the base point of $C_{k}$. We assume that these $m$ paths meet only at $w$, and set $c=\bigcup_{k} c_{k}([0,1]) \subset W$. Transporting $C_{k}$ along $c_{k}$, we obtain a loop in $W$ based at $w$ and representing some $x_{k} \in \pi=\pi_{1}(W, w)$.

Let $X=K(G, 1)$ with base point $x$. Fix a homomorphism $g: \pi \rightarrow G$ and consider a map $\tilde{g}:(W, c) \rightarrow(X, x)$ such that $\tilde{g}_{\#}=g: \pi \rightarrow G=\pi_{1}(X, x)$. For $k=1, \ldots, m$, the $X$-curve $\left(C_{k},\left.\tilde{g}\right|_{C_{k}}\right)$ represents $g\left(x_{k}\right) \in G$. 
Fix a set $\gamma=\left\{\gamma_{1}, \ldots, \gamma_{m}\right\} \subset G^{\prime}$ such that $\gamma_{k} \in q^{-1}\left(g\left(x_{k}\right)\right)$ for all $k$. By Section 4.5 , the vector $\gamma_{k} \in B_{g\left(x_{k}\right)}$ projects to a vector $\left[\gamma_{k}\right] \in A_{C_{k}}^{\circ}$. Set

$$
[\gamma]=\bigotimes_{k=1}^{m}\left[\gamma_{k}\right] \in A_{\partial W}^{\circ}=\bigotimes_{k=1}^{m} A_{C_{k}}^{\circ} .
$$

If $m=0$, then $\gamma=\emptyset$ and by definition $[\gamma]=1_{K} \in K=A_{\emptyset}^{\circ}=A_{\partial W}^{\circ}$.

Lemma 5.1. The homomorphism $\tau^{\circ}(-W, \tilde{g}): A_{\partial W}^{\circ} \rightarrow K$ associated with the $X$-cobordism $(-W, \partial W, \emptyset, \tilde{g})$ is computed on $[\gamma]$ by

$$
\tau^{\circ}(-W, \tilde{g})([\gamma])=|\Gamma|^{\chi(W)-1} \times\left|S_{*}(g, \gamma)\right|,
$$

where $S_{*}(g, \gamma)$ is the (finite) set of all pairs (a homomorphism $g^{\prime}: \pi \rightarrow G^{\prime}$, a family $\left.\left\{a_{k} \in \Gamma\right\}_{k=1}^{m}\right)$ such that $q g^{\prime}=g$ and $g^{\prime}\left(x_{k}\right)=a_{k} \gamma_{k} a_{k}^{-1}$ for all $k$.

Proof. Take a CW-decomposition $T$ of $W$ having the base points of $C_{1}, \ldots, C_{m}$ and $w$ as the only vertices, having $C_{1}, \ldots, C_{m}$ and $c_{1}, \ldots, c_{m}$ among edges, and having only one face. Then $\chi(W)=m+2-r$, where $r$ is the number of edges of $T$. The map $\tilde{g}$ is represented by a $G$-system on $T$ assigning 1 to $c_{k}$ and $g\left(x_{k}\right)$ to $C_{k}$ for all $k$. The computation of $\eta_{B}$ and $\left\{b_{\alpha}\right\}_{\alpha \in G}$ at the end of Section 4.1 implies that $\tau^{\circ}(-W, \tilde{g})([\gamma])=|\Gamma| \times|\Gamma|^{-(r-m)} \times\left(\right.$ the number of $G^{\prime}$-systems on $T$ which are lifts of our $G$-system and which assign $\gamma_{k}$ to $C_{k}$ for all $k$ ). The latter number is equal to $\left|\oint_{*}(g, \gamma)\right|$.

Lemma 5.2. Suppose that the genus of $W$ is positive and let $H=\left\langle x_{1}, \ldots, x_{m}\right\rangle$ be the subgroup of $\pi$ (freely) generated by $x_{1}, \ldots, x_{m}$. If $g: \pi \rightarrow G$ is an epimorphism whose restriction to $H$ is injective, then the set $\gamma=\left\{\gamma_{1}, \ldots, \gamma_{m}\right\} \subset G^{\prime}$ is q-free in the sense of Section 2 and

$$
\tau^{\circ}(-W, \tilde{g})([\gamma])=\sum_{\rho \in I_{0}(q)}(\operatorname{dim} \rho)^{\chi(W)} g^{*}\left(\zeta^{\rho, \gamma}\right)([W, \partial W]) \prod_{k=1}^{m} t_{\rho}\left(\gamma_{k}\right) .
$$

The first claim of the lemma follows from the equality $\langle q(\gamma)\rangle=g(H)$. Note that $\zeta^{\rho, \gamma} \in H^{2}\left(G, g(H) ; K^{*}\right)$ and $g^{*}\left(\zeta^{\rho, \gamma}\right) \in H^{2}\left(\pi, H ; K^{*}\right)$. We have

$$
H_{2}(\pi, H ; \mathbb{Z})=H_{2}(W, c \cup \partial W ; \mathbb{Z})=H_{2}(W, \partial W ; \mathbb{Z})=\mathbb{Z} \cdot[W, \partial W]
$$

so that we can evaluate $g^{*}\left(\zeta^{\rho, \gamma}\right)$ on $[W, \partial W]$. For the definition of $I_{0}(q)$, see Section 2.1 .

Lemma 5.2 will be proven in Section 10. Combining Lemmas 5.1 and 5.2, we obtain that for any $q$-free system $\gamma=\left\{\gamma_{k} \in q^{-1}\left(g\left(x_{k}\right)\right)\right\}_{k=1}^{m}$,

$$
\left|S_{*}(g, \gamma)\right|=|\Gamma| \sum_{\rho \in I_{0}(q)}(|\Gamma| / \operatorname{dim} \rho)^{-\chi(W)} g^{*}\left(\zeta^{\rho, \gamma}\right)([W, \partial W]) \prod_{k=1}^{m} t_{\rho}\left(\gamma_{k}\right) \text {. }
$$


5.2. Proof of Theorem 1.2. We substitute (7) and $g=i d: \pi \rightarrow \pi$ in (16). Since the genus of $W$ is positive, $x_{1}, \ldots, x_{m} \in \pi$ generate a free group of rank $m$. Therefore the set $\gamma \subset \pi^{\prime}$ constructed before the statement of Theorem 1.1 is $q$-free. Theorem 1.2 now follows from (16) provided we show that $\left|S\left(p, s_{\partial}\right)\right|=\mid S_{*}($ id, $\gamma) \mid$.

A pointed section $s$ of $p: E \rightarrow W$ extending $s_{\partial}: \partial W \rightarrow E$ induces a homomorphism $s_{\#}: \pi \rightarrow \pi^{\prime}$ such that $q s_{\#}=$ id. For $k=1, \ldots, m$, the path $\tilde{c}_{k}:[0,1] \rightarrow E$ used in the definition of $\gamma_{k}$ in Section 1 and the path $s c_{k}$ have the same endpoints and both project to $c_{k}$. Let $a_{k} \in \Gamma=\Phi$ be represented by the loop $\left(s c_{k}\right) \tilde{c}_{k}^{-1}:[0,1] \rightarrow E$. Clearly, $\left(s_{\#},\left\{a_{k}\right\}_{k=1}^{m}\right) \in \mathcal{S}_{*}(\mathrm{id}, \gamma)$. The resulting mapping $\delta\left(p, s_{\partial}\right) \rightarrow \mathcal{S}_{*}(\mathrm{id}, \gamma)$ is bijective, as can be easily shown using the same CW-decomposition of $W$ as in the proof of Lemma 5.1. The injectivity results from the identification of bubble equivalent sections in $S\left(p, s_{\partial}\right)$. The surjectivity is obtained by constructing an appropriate section over the 1-skeleton of $W$ and extending it over the face using the injectivity of the inclusion homomorphism $\pi_{1}(F, e) \rightarrow \pi^{\prime}$, where $F=p^{-1}(w)$. Thus, $\left|\mathcal{S}\left(p, s_{\partial}\right)\right|=\left|\mathcal{S}_{*}(\mathrm{id}, \gamma)\right|$.

\section{Crossed $G$-algebras}

We introduce crossed $G$-algebras which will be our main tools in the study of 2dimensional HQFTs with target $K(G, 1)$.

6.1. Basics. We use terminology of Section 4.1. A crossed $G$-algebra is a triple (a $G$-algebra $L=\bigoplus_{\alpha \in G} L_{\alpha}$, an inner product $\eta$ on $L$, a homomorphism $\alpha \mapsto \varphi_{\alpha}$ from $G$ to the group of algebra automorphisms of $L$ ) such that for all $\alpha, \beta \in G$,

(1) $\varphi_{\alpha}\left(L_{\beta}\right) \subset L_{\alpha \beta \alpha^{-1}}$ and $\left.\varphi_{\alpha}\right|_{L_{\alpha}}=\mathrm{id}$;

(2) for any $a \in L_{\alpha}, b \in L$, we have $\varphi_{\alpha}(b) a=a b$;

(3) $\eta\left(\varphi_{\alpha}(a), \varphi_{\alpha}(b)\right)=\eta(a, b)$ for all $a, b \in L$;

(4) for any $c \in L_{\alpha \beta \alpha^{-1} \beta^{-1}}$, the homomorphism $\mu_{c}: L \rightarrow L, x \mapsto c x$ satisfies

$$
\operatorname{Tr}\left(\mu_{c} \varphi_{\beta}: L_{\alpha} \rightarrow L_{\alpha}\right)=\operatorname{Tr}\left(\varphi_{\alpha-1} \mu_{c}: L_{\beta} \rightarrow L_{\beta}\right) .
$$

Axiom (2) implies that $L_{1} \subset L$ lies in the center of $L$. In particular, $L_{1}$ is a commutative associative $K$-algebra with unit. The group $G$ acts on $L_{1}$ by algebra automorphisms. This action determines the dimensions of all $L_{\alpha}$ : applying (4) to $\beta=1$ and $c=1_{L} \in L_{1}$, we obtain

$\operatorname{Dim} L_{\alpha}=\operatorname{Tr}\left(\mathrm{id}: L_{\alpha} \rightarrow L_{\alpha}\right)=\operatorname{Tr}\left(\varphi_{1}: L_{\alpha} \rightarrow L_{\alpha}\right)=\operatorname{Tr}\left(\varphi_{\alpha^{-1}}: L_{1} \rightarrow L_{1}\right)$.

Isomorphisms of crossed $G$-algebras are algebra isomorphisms preserving the $G$-grading and the inner product, and commuting with the action of $G$.

The direct sum $L \oplus L^{\prime}$ of two crossed $G$-algebras $L, L^{\prime}$ is the crossed $G$-algebra defined by $\left(L \oplus L^{\prime}\right)_{\alpha}=L_{\alpha} \oplus L_{\alpha}^{\prime}$ for $\alpha \in G$ with coordinate-wise multiplication, 
inner product, and action of $G$. For $k \in K^{*}$, the $k$-rescaling transforms a crossed $G$-algebra $(L, \eta, \varphi)$ into the crossed $G$-algebra $(L, k \eta, \varphi)$.

6.2. Example. Let $\theta=\left\{\theta_{\alpha, \beta}\right\}_{\alpha, \beta \in G}$ be a normalized $K^{*}$-valued 2-cocycle of $G$. The cocycle identity $\theta_{\alpha, \beta} \theta_{\alpha \beta, \gamma}=\theta_{\alpha, \beta \gamma} \theta_{\beta, \gamma}$ for $\alpha, \beta, \gamma \in G$ and the normalization condition $\theta_{1,1}=1$ imply that $\theta_{1, \alpha}=\theta_{\alpha, 1}=1$ and $\theta_{\alpha, \alpha^{-1}}=\theta_{\alpha^{-1, \alpha}}$ for all $\alpha$.

We define a crossed $G$-algebra $L=L^{\theta}$ as follows. For $\alpha \in G$, let $L_{\alpha}=K l_{\alpha}$ be the one-dimensional vector space generated by a vector $l_{\alpha}$. Multiplication in $L=\bigoplus_{\alpha} L_{\alpha}$ is defined by $l_{\alpha} l_{\beta}=\theta_{\alpha, \beta} l_{\alpha \beta}$. This makes $L$ into an associative algebra with unit $1_{L}=l_{1} \in L_{1}$. The inner product on $L$ is defined by $\eta\left(l_{\alpha}, l_{\alpha^{-1}}\right)=\theta_{\alpha, \alpha^{-1}}$ for all $\alpha \in G$. The action of $\alpha \in G$ is defined on the basis $\left\{l_{\beta}\right\}_{\beta}$ of $L$ by

$$
\varphi_{\alpha}\left(l_{\beta}\right)=\theta_{\alpha, \alpha^{-1}}^{-1} \theta_{\alpha, \beta} \theta_{\alpha \beta, \alpha^{-1}} l_{\alpha \beta \alpha^{-1}} .
$$

It is clear that $\varphi_{\alpha}$ is the only $K$-linear automorphism of $L$ satisfying Axioms (1) and (2). The other axioms of a crossed $G$-algebra are verified by direct computations using solely the cocycle identity and the normalization condition.

Note that the isomorphism class of $L^{\theta}$ depends only on the cohomology class of $\theta$. For $k \in K^{*}$, denote $L^{\theta, k}$ the crossed $G$-algebra obtained from $L^{\theta}$ by $k$-rescaling. If $\theta=1$, then this yields a structure of a crossed $G$-algebra on the group ring $K[G]$.

6.3. Transfer. Let $H$ be a subgroup of $G$ of finite index. A crossed $H$-algebra $\left(L=\bigoplus_{\alpha \in H} L_{\alpha}, \eta, \varphi\right)$ gives rise to a crossed $G$-algebra $\left(\tilde{L}=\bigoplus_{\alpha \in G} \tilde{L}_{\alpha}, \tilde{\eta}, \tilde{\varphi}\right)$ called its transfer and defined as follows. Let $H \backslash G$ be the set of right cosets of $H$ in $G$. For each $i \in H \backslash G$, fix a representative $\omega_{i} \in G$ so that $i=H \omega_{i}$. (It is convenient but not necessary to take $\omega_{i}=1$ for $i=H$.) For $\alpha \in G$, set

$$
N(\alpha)=\left\{i \in H \backslash G \mid \omega_{i} \alpha \omega_{i}^{-1} \in H\right\} \quad \text { and } \quad \tilde{L}_{\alpha}=\bigoplus_{i \in N(\alpha)} L_{\omega_{i} \alpha \omega_{i}^{-1}} .
$$

In particular, if $\alpha$ is not conjugate to elements of $H$, then $\tilde{L}_{\alpha}=0$.

We provide $\tilde{L}=\bigoplus_{\alpha} \tilde{L}_{\alpha}$ with coordinate-wise multiplication. Thus, for $\alpha, \beta \in$ $G$, the multiplication $\tilde{L}_{\alpha} \times \tilde{L}_{\beta} \rightarrow \tilde{L}_{\alpha \beta}$ restricted to $L_{\omega_{i} \alpha \omega_{i}^{-1}} \times L_{\omega_{j} \beta \omega_{j}^{-1}}$ is 0 if $i \neq j$, and is induced by multiplication in $L$

$$
L_{\omega_{i} \alpha \omega_{i}^{-1}} \times L_{\omega_{i} \beta \omega_{i}^{-1}} \rightarrow L_{\omega_{i} \alpha \beta \omega_{i}^{-1}}
$$

if $i=j \in N(\alpha) \cap N(\beta)$. By definition, the algebra $\tilde{L}_{1}$ is a direct sum of $[G: H]$ copies of $L_{1}$ numerated by the elements of $H \backslash G$. The corresponding sum of copies of $1_{L} \in L_{1}$ is the unit of $\tilde{L}_{1}$. The inner product $\tilde{\eta}$ on $\tilde{L}$ is defined by

$$
\left.\tilde{\eta}\right|_{\tilde{L}_{\alpha} \otimes \tilde{L}_{\alpha-1}}=\left.\bigoplus_{i \in N(\alpha)=N\left(\alpha^{-1}\right)} \eta\right|_{\omega_{i} \alpha \omega_{i}^{-1}} \otimes L_{\omega_{i} \alpha^{-1} \omega_{i}^{-1}} .
$$

We now define $\tilde{\varphi}$. The group $G$ acts on $H \backslash G$ by $\alpha(j)=j \alpha^{-1}$ for $\alpha \in G$, $j \in H \backslash G$. The equality $H \omega_{\alpha(j)}=H \omega_{j} \alpha^{-1}$ implies that $\alpha_{j}=\omega_{\alpha(j)} \alpha \omega_{j}^{-1} \in H$. 
Note that the fixed point set of the map $H \backslash G \rightarrow H \backslash G, j \mapsto \alpha(j)$ is $N(\alpha)$. Therefore for any $\beta \in G$, this map sends $N(\beta)$ bijectively onto $N\left(\alpha \beta \alpha^{-1}\right)$. For $j \in N(\beta)$, consider the homomorphism

$$
\varphi_{\alpha_{j}}: L_{\omega_{j} \beta \omega_{j}^{-1}} \rightarrow L_{\alpha_{j} \omega_{j} \beta \omega_{j}^{-1} \alpha_{j}^{-1}}=L_{\omega_{\alpha(j)} \alpha \beta \alpha^{-1} \omega_{\alpha(j)}^{-1}} .
$$

We define $\tilde{\varphi}_{\alpha}: \tilde{L}_{\beta} \rightarrow \tilde{L}_{\alpha \beta \alpha^{-1}}$ to be the direct sum of these homomorphisms over all $j \in N(\beta)$. The identity $\left(\alpha \alpha^{\prime}\right)_{j}=\alpha_{\alpha^{\prime}(j)} \alpha_{j}^{\prime}$ for any $\alpha, \alpha^{\prime} \in G$ implies that $\tilde{\varphi}_{\alpha \alpha^{\prime}}=\tilde{\varphi}_{\alpha} \tilde{\varphi}_{\alpha^{\prime}}$. Hence $\tilde{\varphi}$ is an action of $G$ on $\tilde{L}$.

Lemma 6.1. The triple $(\tilde{L}, \tilde{\eta}, \tilde{\varphi})$ is a crossed $G$-algebra.

Proof. That $\tilde{\eta}$ is an inner product preserved by $\tilde{\varphi}$ follows directly from the definitions and properties of $L$. We check Axioms (1), (2), (4) of Section 6.1.

For $j \in N(\alpha)$, we have $\alpha(j)=j$ and $\alpha_{j}=\omega_{j} \alpha \omega_{j}^{-1}$. By Axiom (1) for $L$, the homomorphism (18) with $\beta=\alpha$ is the identity. This yields Axiom (1) for $\tilde{L}$.

Let $a \in L_{\omega_{i} \alpha \omega_{i}^{-1}} \subset \tilde{L}_{\alpha}, b \in L_{\omega_{j} \beta \omega_{j}^{-1}} \subset \tilde{L}_{\beta}$ with $i \in N(\alpha), j \in N(\beta)$. Then

$$
\tilde{\varphi}_{\alpha}(b)=\varphi_{\alpha_{j}}(b) \in L_{\omega_{\alpha(j)} \alpha \beta \alpha^{-1} \omega_{\alpha(j)}^{-1}} .
$$

If $i \neq j$ then $\alpha(j) \neq \alpha(i)=i$ and $\tilde{\varphi}_{\alpha}(b) a=0=a b$. If $i=j$, then $\alpha_{j}=\alpha_{i}=$ $\omega_{i} \alpha \omega_{i}^{-1}$. By Axiom (2) for $L$,

$$
\tilde{\varphi}_{\alpha}(b) a=\varphi_{\alpha_{j}}(b) a=\varphi_{\omega_{i} \alpha \omega_{i}^{-1}}(b) a=a b .
$$

We now check formula (17) with $L, \varphi$ replaced by $\tilde{L}, \tilde{\varphi}$. Since both sides are linear functions of $c$, it suffices to treat the case where

$$
c \in L_{\omega_{i} \alpha \beta \alpha^{-1} \beta^{-1} \omega_{i}^{-1}} \subset \tilde{L}_{\alpha \beta \alpha^{-1} \beta^{-1}}
$$

with $i \in N\left(\alpha \beta \alpha^{-1} \beta^{-1}\right)$. A direct application of definitions shows that both sides of the desired formula are equal to 0 unless $i \in N(\alpha) \cap N(\beta)$. If $i \in N(\alpha) \cap N(\beta)$ then the trace of $\mu_{c} \tilde{\varphi}_{\beta}: \tilde{L}_{\alpha} \rightarrow \tilde{L}_{\alpha}$ is equal to the trace of the endomorphism $\mu_{c} \varphi_{\omega_{i} \beta \omega_{i}^{-1}}$ of $\tilde{L}_{\omega_{i} \alpha \omega_{i}^{-1}}$. The trace of $\tilde{\varphi}_{\alpha^{-1}} \mu_{c}: \tilde{L}_{\beta} \rightarrow \tilde{L}_{\beta}$ is equal to the trace of the endomorphism $\varphi_{\omega_{i} \alpha^{-1} \omega_{i}^{-1}} \mu_{c}$ of $\tilde{L}_{\omega_{i} \beta \omega_{i}^{-1}}$. The equality of these two traces follows from Axiom (4) for $L$.

It follows from Lemma 8.4 below that the isomorphism class of $(\tilde{L}, \tilde{\eta}, \tilde{\varphi})$ does not depend on the choice of the representatives $\left\{\omega_{i}\right\}_{i}$. A direct algebraic proof is left to the reader as an exercise.

We can apply transfer to the crossed $H$-algebra $L^{\theta}$ derived from a $K^{*}$-valued normalized 2-cocycle $\theta$ on $H$. The resulting crossed $G$-algebra is denoted $L^{G, H, \theta}$. For $k \in K^{*}$, denote $L^{G, H, \theta, k}$ the crossed $G$-algebra obtained from $L^{G, H, \theta}$ by $k$-rescaling. It is clear that transfer and rescaling commute so that $L^{G, H, \theta, k}$ is the transfer of the crossed $H$-algebra $L^{\theta, k}$. 
6.4. Semisimple $\boldsymbol{G}$-algebras. A crossed $G$-algebra $L=\bigoplus_{\alpha \in G} L_{\alpha}$ is semisimple if $L_{1}$ is a direct sum of several mutually annihilating (and therefore mutually orthogonal) copies of $K$. For instance, the crossed $G$-algebra $L^{\theta}$ in Example 6.2 is semisimple since $L_{1}=K$. Direct sums, rescalings, and transfers of semisimple crossed group-algebras are semisimple.

If a crossed $G$-algebra $L$ is semisimple, then $L_{1}$ has a basis $I$ such that $i j=\delta_{j}^{i} i$ for all $i, j \in I$, where $\delta_{j}^{i}$ is the Kronecker delta. Such a basis is unique and denoted bas $(L)$. Its elements are called the basic idempotents of $L$. Algebra automorphisms of $L_{1}$ preserve bas $(L)$ set-wise. In particular, the action of $G$ on $L$ restricts to an action of $G$ on bas $(L)$. We call $L$ simple if (it is semisimple and) the action of $G$ on bas $(L)$ is transitive. The crossed $G$-algebra $L^{\theta}$ in Example 6.2 is simple because bas $\left(L^{\theta}\right)$ is a one point set. The crossed $G$-algebra $L=L^{G, H, \theta, k}$ constructed at the end of Section 6.3 is simple because $L_{1}$ is a direct sum of copies of $L_{1}^{\theta}=K$ numerated by elements of $H \backslash G$ and the action of $G$ on $L_{1}$ permutes these copies of $K$ via the natural (transitive) action of $G$ on $H \backslash G$.

Consider again a semisimple crossed $G$-algebra $L$ and set $I=\operatorname{bas}(L)$. The equality $1_{L}=\sum_{i \in I} i$ and the fact that $I$ lies in the center of $L$ imply that $L$ splits as a direct sum of mutually annihilating subalgebras $\{i L\}_{i \in I}$ :

$$
L=\bigoplus_{i \in I} i L \quad \text { where } i L=L i=\bigoplus_{\alpha \in G} i L_{\alpha} .
$$

The subalgebras $\{i L\}_{i \in I}$ of $L$ are permuted via the action of $G$ on $L$. Therefore for any orbit $\kappa \subset I$ of the action of $G$ on $I$, the vector space $\kappa L=\bigoplus_{i \in \kappa} i L$ is a $G$-invariant subalgebra of $L$. Restricting the action of $G$ and the inner product on $L$ to $\kappa L$ we transform the latter into a simple crossed $G$-algebra. We conclude that each semisimple crossed $G$-algebra canonically splits as a direct sum of simple crossed $G$-algebras.

We now classify simple crossed $G$-algebras. Denote by $\mathcal{A}(G)$ the set of isomorphism classes of pairs (a simple crossed $G$-algebra $L$, a basic idempotent $i \in \operatorname{bas}(L)$ ). (Two such pairs are isomorphic if there is an isomorphism of the crossed $G$-algebras preserving the distinguished basic idempotent.) The group $G$ acts on $\mathcal{A}(G)$ by $\alpha(L, i)=\left(L, \varphi_{\alpha}(i)\right)$ for $\alpha \in G$. Let $\mathscr{B}(G)$ be the set of triples (a subgroup $H \subset G$ of finite index, a cohomology class $\theta \in H^{2}\left(H ; K^{*}\right)$, an element $k$ of $\left.K^{*}\right)$. The group $G$ acts on $\mathcal{B}(G)$ by $\alpha(H, \theta, k)=\left(\alpha H \alpha^{-1}, \alpha_{*}(\theta), k\right)$ where $\alpha \in G$ and $\alpha_{*}: H^{2}\left(H ; K^{*}\right) \rightarrow H^{2}\left(\alpha H \alpha^{-1} ; K^{*}\right)$ is the isomorphism induced by the conjugation by $\alpha$.

Lemma 6.2. There is a canonical G-equivariant bijection $\mathcal{A}(G) \rightarrow \mathscr{B}(G)$.

Proof. Let $L=\bigoplus_{\alpha \in G} L_{\alpha}$ be a simple crossed $G$-algebra and $i \in$ bas $(L)$. Let $G_{i}=\left\{\alpha \in G \mid \varphi_{\alpha}(i)=i\right\}$ be the stabilizer of $i$. We first compute the dimension of $i L_{\alpha} \subset L_{\alpha}$ for all $\alpha \in G$. Applying (17) to $\beta=1$ and $c=i \in L_{1}$, we obtain

$$
\operatorname{dim}\left(i L_{\alpha}\right)=\operatorname{Tr}\left(\mu_{i}: L_{\alpha} \rightarrow L_{\alpha}\right)=\operatorname{Tr}\left(\varphi_{\alpha^{-1}} \mu_{i}: L_{1} \rightarrow L_{1}\right) .
$$


The endomorphism $\varphi_{\alpha^{-1}} \mu_{i}$ of $L_{1}$ carries $i$ to $\varphi_{\alpha^{-1}}(i) \in I$ and carries all other elements of the basis $I$ to 0 . Hence

$$
\operatorname{dim}\left(i L_{\alpha}\right)= \begin{cases}1 & \text { if } \alpha \in G_{i}, \\ 0 & \text { if } \alpha \in G-G_{i}\end{cases}
$$

For every $\alpha \in G_{i}-\{1\}$, pick a non-zero vector $s_{\alpha} \in i L_{\alpha}$. For $\alpha=1$, set $s_{\alpha}=$ $i \in i L_{1}$. Then for any $\alpha, \beta \in G_{i}$, we have $s_{\alpha} s_{\beta}=\nabla_{\alpha, \beta} s_{\alpha \beta}$ with $\nabla_{\alpha, \beta} \in K$. We claim that $\nabla_{\alpha, \beta} \neq 0$. Indeed, the inner product $\eta$ in $L$ satisfies $\eta\left(s_{\beta} s_{\beta-1}, 1_{L}\right)=$ $\eta\left(s_{\beta}, s_{\beta^{-1}}\right) \neq 0$. Hence $s_{\beta} s_{\beta^{-1}}=\nabla s_{1}=\nabla i$ with $\nabla \in K^{*}$ and

$$
\left(s_{\alpha} s_{\beta}\right) s_{\beta^{-1}}=s_{\alpha}\left(s_{\beta} s_{\beta^{-1}}\right)=\nabla s_{\alpha} i=\nabla s_{\alpha} \neq 0 .
$$

Therefore $s_{\alpha} s_{\beta} \neq 0$ and $\nabla_{\alpha, \beta} \neq 0$.

The associativity of multiplication in $L$ and the choice $s_{1}=i$ ensure that $\left\{\nabla_{\alpha, \beta}\right\}_{\alpha, \beta}$ is a normalized $K^{*}$-valued 2-cocycle on $G_{i}$. Let $\nabla_{i} \in H^{2}\left(G_{i} ; K^{*}\right)$ be its cohomology class. Under a different choice of $\left\{s_{\alpha}\right\}_{\alpha}$ we obtain the same $\nabla_{i}$.

The formula $(L, i) \mapsto\left(G_{i}, \nabla_{i}, \eta(i, i)\right)$ defines a $G$-equivariant mapping $\mathcal{A}(G) \rightarrow$ $\mathscr{B}(G)$. In the opposite direction, any triple $(H, \theta, k) \in \mathscr{B}(G)$ determines a simple crossed $G$-algebra $\tilde{L}=L^{G, H, \theta, k}$. The elements of bas $(\tilde{L})$ bijectively correspond to the elements of $H \backslash G$. Let $i_{\theta} \in \operatorname{bas}(\tilde{L})$ correspond to $H \backslash H \in H \backslash G$. The formula $(H, \theta, k) \mapsto\left(\tilde{L}, i_{\theta}\right)$ defines a mapping $\mathcal{B}(G) \rightarrow \mathcal{A}(G)$.

We claim that these mappings $\mathscr{B}(G) \rightarrow \mathcal{A}(G)$ and $\mathcal{A}(G) \rightarrow \mathscr{B}(G)$ are mutually inverse bijections. Let us verify that the composition $\mathscr{B}(G) \rightarrow \mathcal{A}(G) \rightarrow \mathscr{B}(G)$ is the identity. Consider the pair $\left(\tilde{L}, i_{\theta}\right)$ derived as above from $(H, \theta, k) \in \mathscr{B}(G)$. The stabilizer of $i_{\theta}=H \backslash H \in H \backslash G=$ bas $(\tilde{L})$ with respect to the natural action of $G$ is $H$. It follows from the definition of $\tilde{L}$ that $i_{\theta} \tilde{L}_{\alpha}=L_{\alpha}^{\theta}$ for all $\alpha \in H$. Set $s_{\alpha}=l_{\alpha} \in L_{\alpha}^{\theta}=i_{\theta} \tilde{L}_{\alpha}$, where $l_{\alpha}$ is the vector used in the definition of $L^{\theta}$. Now, it is obvious that the mapping $\mathcal{A}(G) \rightarrow \mathcal{B}(G)$ carries $\left(\tilde{L}, i_{\theta}\right)$ to $(H, \theta, k)$.

To accomplish the proof, it suffices to show that the mapping $\mathcal{A}(G) \rightarrow \mathscr{B}(G)$ is injective. It is enough to prove that a simple crossed $G$-algebra $L$ with distinguished basic idempotent $i_{0} \in L_{1}$ can be uniquely reconstructed from the triple (the stabilizer $H \subset G$ of $i_{0}$, the $H$-algebra $i_{0} L$, the element $k=\eta\left(i_{0}, i_{0}\right)$ of $K$ ). Recall that $L=\bigoplus_{i \in I} i L$, where $I=\operatorname{bas}(L)$. Since the action of $G$ on $I$ is transitive, for each $i \in I$ there is $\omega_{i} \in G$ such that $\varphi_{\omega_{i}}\left(i_{0}\right)=i$. We take $\omega_{i_{0}}=1$. The isomorphism $\varphi_{\omega_{i}}: L \rightarrow L$ maps $i_{0} L$ bijectively onto $i L$. Pick a non-zero vector $s_{\alpha}$ in $i_{0} L_{\alpha} \cong K$ for all $\alpha \in H$. Then the set $\left\{\varphi_{\omega_{i}}\left(s_{\alpha}\right) \mid i \in I, \alpha \in H\right\}$ is a basis of $L$. Multiplication in $L$ is computed in this basis by

$$
\varphi_{\omega_{i}}\left(s_{\alpha}\right) \varphi_{\omega_{i^{\prime}}}\left(s_{\alpha^{\prime}}\right)= \begin{cases}\varphi_{\omega_{i}}\left(s_{\alpha} s_{\alpha^{\prime}}\right) & \text { if } i=i^{\prime}, \\ 0 & \text { if } i \neq i^{\prime} .\end{cases}
$$

The inner product $\eta$ on $L$ is determined by the algebra structure of $L$ and the equalities $\eta\left(i, 1_{L}\right)=\eta(i, i)=k$ for all $i \in I$. It remains to recover the action $\varphi$ of $G$ 
on $L$. Given $\alpha \in H$, the homomorphism $\varphi_{\alpha}: i_{0} L \rightarrow i_{0} L$ is fully determined by the condition $\varphi_{\alpha}\left(s_{\beta}\right) s_{\alpha}=s_{\alpha} s_{\beta}$ for all $\beta \in H$ (we use that $s_{\alpha \beta \alpha^{-1}} s_{\alpha} \neq 0$, cf. (19)). Each $\beta \in G$ expands as a product $\omega_{i} \alpha$ where $i=\varphi_{\beta}\left(i_{0}\right)$ and $\alpha \in H$. This gives $\varphi_{\beta}=\varphi_{\omega_{i}} \varphi_{\alpha}$ and computes the restriction of $\varphi_{\beta}$ to $i_{0} L$. Knowing these restrictions for all $\beta \in G$, we can recover $\varphi_{\beta}: L \rightarrow L$ by $\varphi_{\beta}\left(\varphi_{\omega_{i}}\left(s_{\alpha}\right)\right)=\varphi_{\beta \omega_{i}}\left(s_{\alpha}\right)$.

\section{The underlying $G$-algebra}

We study a two-dimensional $X$-HQFT $(A, \tau)$ where $X=K(G, 1)$ with base point $x$. We derive from $(A, \tau)$ an "underlying" crossed $G$-algebra.

7.1. Conventions. Given an oriented surface $W$ and a component $M$ of $\partial W$, we write $M_{+}$(resp. $M_{-}$) for $M$ with orientation induced from that in $W$ (resp. in $-W$ ). When all components $M$ of $\partial W$ are labeled with signs $\varepsilon(M)= \pm$, we have in the category of oriented manifolds $\partial W=\sum_{M} \varepsilon(M) M_{\varepsilon(M)}$. We view such a $W$ as a cobordism whose bottom (resp. top) base is formed by the boundary components labeled with $-($ resp. with + ).

7.2. Disks with holes. We describe the structures of $X$-cobordisms on annuli and disks with two holes. Set $C=S^{1} \times[0,1]$. Fix once and for all an orientation in $C$. Set $C^{0}=S^{1} \times 0 \subset \partial C$ and $C^{1}=S^{1} \times 1 \subset \partial C$. It is convenient to think of $C$ as of an annulus in $\mathbb{R}^{2}$ with clockwise orientation such that $C^{0}$ (resp. $C^{1}$ ) is its internal (resp. external) boundary component. We provide $C^{0}, C^{1}$ with base points $s \times 0$, $s \times 1$, respectively, where $s \in S^{1}$. Given $\varepsilon, \mu= \pm 1$, denote by $C_{\varepsilon, \mu}$ the oriented annulus $C$ with oriented pointed boundary $C_{\varepsilon}^{0} \cup C_{\mu}^{1}$. For example, if $C$ lies in $\mathbb{R}^{2}$ as above, then both components of $\partial C_{-+}$are oriented clockwise.

The homotopy class of a map $g: C_{\varepsilon, \mu} \rightarrow X$ is determined by the elements $\alpha, \beta$ of $G$, represented by the loops $\left.g\right|_{C_{\varepsilon}^{0}}$ and $\left.g\right|_{s \times[0,1]}$, respectively. Here [0,1] is oriented from 0 to 1 . Note that $\left.g\right|_{C_{\mu}^{1}}$ represents $\beta^{-1} \alpha^{-\varepsilon \mu} \beta \in G$. The $X$-surface $\left(C_{\varepsilon, \mu}\right.$, the map $C_{\varepsilon, \mu} \rightarrow X$ corresponding to $\left.\alpha, \beta \in G\right)$ is denoted $C_{\varepsilon, \mu}(\alpha ; \beta)$; see Figure 1 .
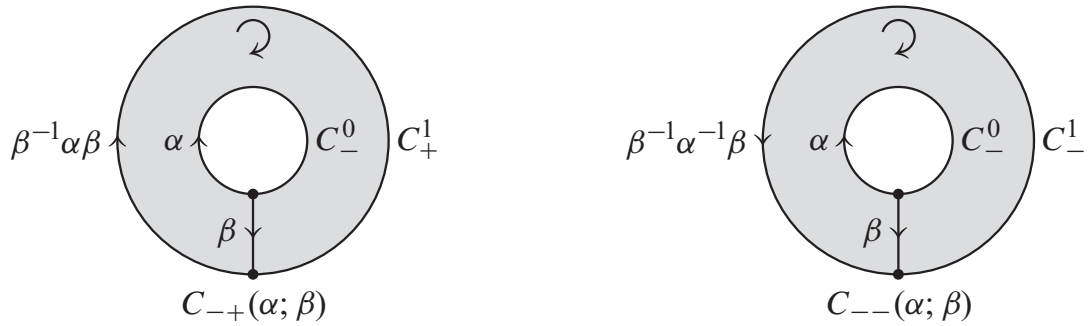

Figure 1 The $X$-annuli $C_{-+}(\alpha ; \beta)$ and $C_{--}(\alpha ; \beta)$. 
Let $D$ be a 2-disk with two holes. Let $Y, Z, T$ be the boundary components of $D$ with base points $y, z, t$, respectively. Fix proper embedded arcs $t y$ and $t z$ in $D$ oriented from $t$ to $y, z$ and meeting solely at $t$. We provide $D$ with the orientation obtained by rotating $t y$ towards $t z$ in $D$ around $t$. One can view $D$ as a disk with two holes in $\mathbb{R}^{2}$ with clockwise orientation such that $Y, Z$ are the internal boundary components, $T$ is the external boundary component, and $y, z, t$ are the bottom points of $Y, Z, T$. Given $\varepsilon, \mu, \nu= \pm$, let $D_{\varepsilon, \mu, \nu}$ be $D$ with oriented pointed boundary $Y_{\varepsilon} \cup Z_{\mu} \cup T_{\nu}$. For example, if $D$ lies in $\mathbb{R}^{2}$ as above, then all components of $\partial D_{--+}$ are oriented clockwise.

To a map $g: D_{\varepsilon, \mu, \nu} \rightarrow X$ we assign the homotopy classes of the loops $\left.g\right|_{Y_{\varepsilon}}$, $\left.g\right|_{Z_{\mu}},\left.g\right|_{t y},\left.g\right|_{t z}$. This gives a bijection from the set of homotopy classes of maps $D_{\varepsilon, \mu, \nu} \rightarrow X$ onto $G^{4}$. For a tuple $(\alpha, \beta, \rho, \delta) \in G^{4}$, denote $D_{\varepsilon, \mu, v}(\alpha, \beta ; \rho, \delta)$ the disk with holes $D_{\varepsilon, \mu, \nu}$ endowed with the map $g$ to $X$ corresponding to this tuple, cf. Figure 2. Note that the loops $\left.g\right|_{Y_{\varepsilon}},\left.g\right|_{Z_{\mu}},\left.g\right|_{T_{v}}$ represent $\alpha, \beta, \gamma=\left(\rho \alpha^{-\varepsilon} \rho^{-1} \delta \beta^{-\mu} \delta^{-1}\right)^{v}$, respectively. As an exercise, the reader may construct an $X$-homeomorphism

$$
D_{\varepsilon, \mu, \nu}(\alpha, \beta ; \rho, \delta) \approx D_{\mu, \nu, \varepsilon}\left(\beta, \gamma ; \rho^{-1} \delta, \rho^{-1}\right)
$$

cyclically permuting the components $Y, Z, T$ of $\partial D$.

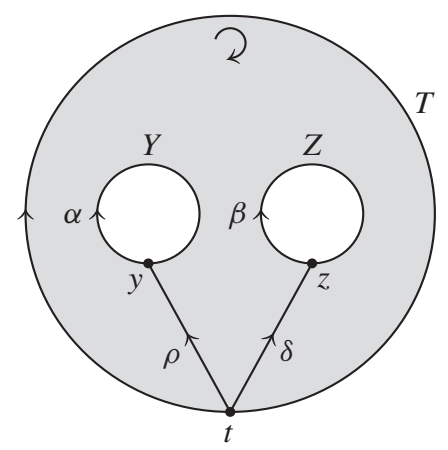

Figure 2 The $X$-disk with holes $D_{--+}(\alpha, \beta ; \rho, \delta)$.

7.3. Algebra $L$. A connected $X$-curve $M$ (i.e., a connected 1-dimensional $X$-manifold) represents an element $\alpha=\alpha(M)$ of $\pi_{1}(X, x)=G$, cf. Section 4.5. We shall sometimes write $(M, \alpha)$ for $M$. The vector space $A_{M}$ depends only on $\alpha(M)$ up to canonical isomorphisms. This follows from Lemma 3.1 because any two oriented pointed circles are related by a (unique up to isotopy) orientation-preserving and base point preserving homeomorphism. In this way, for every $\alpha \in G$, the HQFT $(A, \tau)$ gives a finite-dimensional vector space. It is denoted $L_{\alpha}$.

We provide $L=\bigoplus_{\alpha \in G} L_{\alpha}$ with multiplication and inner product as follows. For $\alpha, \beta \in G$, the disk with two holes $D_{--+}(\alpha, \beta ; 1,1)$ is an $X$-cobordism between the 
$X$-curves $\left(Y_{-}, \alpha\right) \amalg\left(Z_{-}, \beta\right)$ and $\left(T_{+}, \alpha \beta\right)$. Consider the associated homomorphism $\tau\left(D_{--+}(\alpha, \beta ; 1,1)\right): L_{\alpha} \otimes L_{\beta} \rightarrow L_{\alpha \beta}$. We define multiplication in $L$ by

$$
a b=\tau\left(D_{--+}(\alpha, \beta ; 1,1)\right)(a \otimes b) \in L_{\alpha \beta}
$$

for $a \in L_{\alpha}, b \in L_{\beta}$. The annulus $C_{--}(\alpha ; 1)$ is an $X$-cobordism between $\left(C_{-}^{0}, \alpha\right) \amalg$ $\left(C_{-}^{1}, \alpha^{-1}\right)$ and $\emptyset$. Set

$$
\eta_{\alpha}=\tau\left(C_{--}(\alpha ; 1)\right): L_{\alpha} \otimes L_{\alpha^{-1}} \rightarrow K .
$$

The properties of HQFTs stated in Section 3.2 imply that $\eta_{\alpha}$ is non-degenerate for all $\alpha$ and $\eta_{\alpha^{-1}}$ is obtained from $\eta_{\alpha}$ by the permutation of the tensor factors. Let $\eta: L \otimes L \rightarrow K$ be the symmetric bilinear form whose restriction to $L_{\alpha} \otimes L_{\beta}$ with $\alpha, \beta \in G$ is zero if $\alpha \beta \neq 1$ and is $\eta_{\alpha}$ if $\alpha \beta=1$.

The group $G$ acts on $L$ as follows. For $\alpha, \beta \in G$, the annulus $C_{-+}\left(\alpha ; \beta^{-1}\right)$ is an $X$-cobordism between $\left(C_{-}^{0}, \alpha\right)$ and $\left(C_{+}^{1}, \beta \alpha \beta^{-1}\right)$. Set

$$
\varphi_{\beta}=\tau\left(C_{-+}\left(\alpha ; \beta^{-1}\right)\right): L_{\alpha} \rightarrow L_{\beta \alpha \beta^{-1}} .
$$

Axioms (3) and (6) of an HQFT yield that $\varphi_{\beta \gamma}=\varphi_{\beta} \varphi_{\gamma}$ for $\beta, \gamma \in G$ and $\varphi_{1}=$ id.

Lemma 7.1. The triple $(L, \eta, \varphi)$ is a crossed $G$-algebra.

Proof. Let us prove that $(a b) c=a(b c)$ for any $a \in L_{\alpha}, b \in L_{\beta}, c \in L_{\gamma}$ with $\alpha, \beta, \gamma \in G$. Consider the $X$-cobordisms $W_{0}=D_{--+}(\alpha, \beta ; 1,1) \amalg C_{-+}(\gamma ; 1)$ and $W_{1}=D_{--+}(\alpha \beta, \gamma ; 1,1)$. Here $W_{0}$ is an $X$-cobordism between $\left(Y_{-}, \alpha\right) \amalg\left(Z_{-}, \beta\right) \amalg$ $\left(C_{-}^{0}, \gamma\right)$ and $\left(T_{+}, \alpha \beta\right) \amalg\left(C_{+}^{1}, \gamma\right)$. By Axioms (4) and (6) of an HQFT and the definition of multiplication in $L$, the homomorphism $\tau\left(W_{0}\right): L_{\alpha} \otimes L_{\beta} \otimes L_{\gamma} \rightarrow L_{\alpha \beta} \otimes L_{\gamma}$ carries $a \otimes b \otimes c$ into $a b \otimes c$. The homomorphism $\tau\left(W_{1}\right): L_{\alpha \beta} \otimes L_{\gamma} \rightarrow L_{\alpha \beta \gamma}$ carries $a b \otimes c$ into $(a b) c$. The gluing of $W_{0}$ to $W_{1}$ along an $X$-homeomorphism $\left(T_{+}, \alpha \beta\right) \amalg\left(C_{+}^{1}, \gamma\right) \approx\left(Y_{-}, \alpha \beta\right) \amalg\left(Z_{-}, \gamma\right)$ yields an $X$-cobordism $W$ and

$$
\tau(W)(a \otimes b \otimes c)=\tau\left(W_{1}\right) \tau\left(W_{0}\right)(a \otimes b \otimes c)=\tau\left(W_{1}\right)(a b \otimes c)=(a b) c .
$$

The same $X$-cobordism $W$ can be also obtained by gluing the $X$-cobordisms $C_{-+}(\alpha ; 1) \amalg D_{--+}(\beta, \gamma ; 1,1)$ and $D_{--+}(\alpha, \beta \gamma ; 1,1)$ along an $X$-homeomorphism

$$
\left(C_{+}^{1}, \alpha\right) \amalg\left(T_{+}, \beta \gamma\right) \approx\left(Y_{-}, \alpha\right) \amalg\left(Z_{-}, \beta \gamma\right) .
$$

Therefore $\tau(W)(a \otimes b \otimes c)=a(b c)$. Thus, $(a b) c=a(b c)$.

The unit of $L$ is constructed as follows. Let $B_{+}$be an oriented 2-disk whose boundary is pointed and endowed with induced orientation. There is only one homotopy class of maps $B_{+} \rightarrow X$. The corresponding homomorphism $\tau\left(B_{+}\right): K \rightarrow L_{1}$ carries $1_{K} \in K$ into an element of $L_{1}$, denoted $1_{L}$. Let us prove that $1_{L}$ is a right unit of $L$ (the proof that it is a left unit is similar). Let $a \in L_{\alpha}$ with $\alpha \in G$. Consider 
the $X$-cobordisms $W_{0}=C_{-+}(\alpha ; 1) \amalg B_{+}$and $W_{1}=D_{--+}(\alpha, 1 ; 1,1)$. The gluing of $W_{0}$ to $W_{1}$ along an $X$-homeomorphism $\left(C_{+}^{1}, \alpha\right) \amalg\left(\partial B_{+}, 1\right) \approx\left(Y_{-}, \alpha\right) \amalg\left(Z_{-}, 1\right)$ yields an $X$-cobordism $X$-homeomorphic to $C_{-+}(\alpha ; 1)$. By Axioms (6) and (3) of an HQFT,

$$
a=\tau\left(C_{-+}(\alpha ; 1)\right)(a)=\tau\left(W_{1}\right) \tau\left(W_{0}\right)(a)=\tau\left(W_{1}\right)\left(a \otimes 1_{L}\right)=a 1_{L} .
$$

To show that $\eta$ is an inner product on $L$, we need only to prove that $\eta(a b, c)=$ $\eta(a, b c)$ for any $a \in L_{\alpha}, b \in L_{\beta}, c \in L_{\gamma}$ with $\alpha, \beta, \gamma \in G$. If $\alpha \beta \gamma \neq 1$, then $\eta(a b, c)=0=\eta(a, b c)$. Assume that $\alpha \beta \gamma=1$. Gluing $C_{--}(\alpha \beta ; 1)$ to $D_{--+}(\alpha, \beta ; 1,1)$ along $\left(C_{-}^{0}, \alpha \beta\right) \approx\left(T_{+}, \alpha \beta\right)$, we obtain $D_{---}(\alpha, \beta ; 1,1)$. Hence

$$
\eta(a b, c)=\tau\left(D_{---}(\alpha, \beta ; 1,1)\right)(a \otimes b \otimes c) .
$$

Similarly, gluing $C_{--}(\alpha ; 1)$ to $D_{--+}(\beta, \gamma ; 1,1)$ along $\left(C_{-}^{1}, \alpha^{-1}\right) \approx\left(T_{+}, \beta \gamma\right)$, we obtain $D_{---}(\beta, \gamma ; 1,1)$. Hence

$$
\eta(a, b c)=\tau\left(D_{---}(\beta, \gamma ; 1,1)\right)(b \otimes c \otimes a) .
$$

By (20) (where $\rho=\delta=1$ ) and Axiom (2) of an HQFT, $\eta(a b, c)=\eta(a, b c)$.

Let us prove that $\varphi_{\rho}: L \rightarrow L$ is an algebra homomorphism for all $\rho \in G$. Pick $a \in L_{\alpha}, b \in L_{\beta}$ with $\alpha, \beta \in G$. Note that for any $\rho, \delta \in G$, the homomorphism

$$
\tau\left(D_{--+}(\alpha, \beta ; \rho, \delta)\right): L_{\alpha} \otimes L_{\beta} \rightarrow L_{\rho \alpha \rho^{-1} \delta \beta \delta^{-1}}
$$

carries $a \otimes b$ to $\varphi_{\rho}(a) \varphi_{\delta}(b)$. This is so because $D_{--+}(\alpha, \beta ; \rho, \delta)$ can be obtained by gluing $C_{-+}\left(\alpha ; \rho^{-1}\right) \amalg C_{-+}\left(\beta ; \delta^{-1}\right)$ to $D_{--+}(\alpha, \beta ; 1,1)$. Similarly, gluing $C_{-+}\left(\alpha \beta ; \rho^{-1}\right)$ to $D_{--+}(\alpha, \beta ; 1,1)$ along an $X$-homeomorphism $\left(C_{-}^{0}, \alpha \beta\right) \approx$ $\left(T_{+}, \alpha \beta\right)$, we obtain $D_{--+}(\alpha, \beta ; \rho, \rho)$. Therefore

$$
\varphi_{\rho}(a b)=\tau\left(D_{--+}(\alpha, \beta ; \rho, \rho)\right)(a \otimes b)=\varphi_{\rho}(a) \varphi_{\rho}(b) .
$$

Let us verify Axioms (1)-(4) of a crossed $G$-algebra.

(1) The Dehn twist along the circle $S^{1} \times(1 / 2) \subset C_{-+}(\alpha ; 1)$ yields an $X$-homeomorphism $C_{-+}(\alpha ; 1) \rightarrow C_{-+}\left(\alpha ; \alpha^{-1}\right)$. Axiom (2) of an HQFT implies that $\left.\varphi_{\alpha}\right|_{L_{\alpha}}=\tau\left(C_{-+}\left(\alpha ; \alpha^{-1}\right)\right)=\tau\left(C_{-+}(\alpha ; 1)\right)=$ id.

(2) Consider a self-homeomorphism $f$ of the disk with two holes $D$ which is the identity on $T$ and permutes $(Y, y)$ and $(Z, z)$. We choose $f$ so that $f(t z)=t y$ and $f(t y)$ is an arc leading from $t$ to $z$ and homotopic to the product of four arcs ty, $\partial Y,(t y)^{-1}, t z$. An easy computation shows that $f$ is an $X$-homeomorphism $D_{--+}(\alpha, \beta ; 1,1) \rightarrow D_{--+}\left(\beta, \alpha ; 1, \beta^{-1}\right)$. Axiom (2) of an HQFT implies that the homomorphism $\tau\left(D_{--+}(\alpha, \beta ; 1,1)\right): L_{\alpha} \otimes L_{\beta} \rightarrow L_{\alpha \beta}$ is obtained from the homomorphism $\tau\left(D_{--+}\left(\beta, \alpha ; 1, \beta^{-1}\right)\right): L_{\beta} \otimes L_{\alpha} \rightarrow L_{\alpha \beta}$ by composing with the flip $L_{\alpha} \otimes L_{\beta} \rightarrow L_{\beta} \otimes L_{\alpha}$. Therefore for any $a \in L_{\alpha}, b \in L_{\beta}$,

$a b=\tau\left(D_{--+}(\alpha, \beta ; 1,1)\right)(a \otimes b)=\tau\left(D_{--+}\left(\beta, \alpha ; 1, \beta^{-1}\right)\right)(b \otimes a)=b \varphi_{\beta^{-1}}(a)$. 
Replacing $a$ with $\varphi_{\alpha}(b)$ and $b$ with $a$, we obtain $\varphi_{\alpha}(b) a=a \varphi_{\alpha^{-1}}\left(\varphi_{\alpha}(b)\right)=a b$.

(3) The identity $\eta\left(\varphi_{\beta}(a), \varphi_{\beta}(b)\right)=\eta(a, b)$ with $a \in L_{\alpha}, b \in L_{\alpha^{-1}}$ follows from the fact that the annulus $C_{--}(\alpha ; 1)$ used to define $\eta_{\alpha}$ may be obtained by gluing the annuli $C_{-+}\left(\alpha ; \beta^{-1}\right), C_{--}\left(\beta \alpha \beta^{-1} ; 1\right)$, and $C_{-+}\left(\alpha^{-1} ; \beta^{-1}\right)$.

(4) Fix an orientation of $S^{1}$ and a point $s \in S^{1}$. Let $P$ be the punctured torus obtained from $S^{1} \times S^{1}$ by removing a small open 2-disk disjoint from $S^{1} \times\{s\}$ and $\{s\} \times S^{1}$. We assume that the circle $\partial P$ meets $S^{1} \times\{s\}$ and $\{s\} \times S^{1}$ precisely at the point $(s, s)$ and we take $(s, s)$ as the base point of $\partial P$. Provide $P$ with orientation induced from the product orientation in $S^{1} \times S^{1}$.

Fix a map $g: P \rightarrow X=K(G, 1)$ such that $g(s, s)=x$ and the restrictions of $g$ to $S^{1} \times\{s\}$ and $\{s\} \times S^{1}$ represent $\alpha, \beta \in G$, respectively. (The orientations on $S^{1} \times\{s\}$, $\{s\} \times S^{1}$ are induced by that of $S^{1}$.) Then the loop $\left.g\right|_{\partial P}:(\partial P)_{-}=-\partial P \rightarrow X$ represents $\alpha \beta \alpha^{-1} \beta^{-1}$. We view $(P, g)$ as an $X$-cobordism between $\left((\partial P)_{-},\left.g\right|_{\partial P}\right)$ and $\emptyset$. We compute the associated homomorphism $\tau(P, g): L_{\alpha \beta \alpha^{-1} \beta^{-1}} \rightarrow K$ in two different ways. Observe first that $(P, g)$ can be obtained from $D_{--+}\left(\alpha \beta \alpha^{-1} \beta^{-1}, \alpha ; 1, \beta\right)$ by gluing the boundary circles $\left(Z_{-}, \alpha\right)$ and $\left(T_{+}, \alpha\right)$ along an $X$-homeomorphism. (These circles give $S^{1} \times\{s\} \subset P$.) The axioms of an HQFT imply that $\tau(P, g)$ carries $c \in L_{\alpha \beta \alpha^{-1} \beta^{-1}}$ to the trace of the homomorphism

$$
L_{\alpha} \rightarrow L_{\alpha}, \quad d \mapsto \tau\left(D_{--+}\left(\alpha \beta \alpha^{-1} \beta^{-1}, \alpha ; 1, \beta\right)\right)(c \otimes d)=c \varphi_{\beta}(d) .
$$

The same pair $(P, g)$ is obtained from $D_{--+}\left(\alpha \beta \alpha^{-1} \beta^{-1}, \beta ; \alpha^{-1}, \alpha^{-1}\right)$ by gluing along $\left(Z_{-}, \beta\right) \approx\left(T_{+}, \beta\right)$. (The circles $Z_{-}$and $T_{+}$give $\{s\} \times S^{1} \subset P$.) Therefore $\tau(P, g)$ carries $c \in L_{\alpha \beta \alpha^{-1} \beta^{-1}}$ to the trace of the homomorphism

$$
L_{\beta} \rightarrow L_{\beta}, \quad d \mapsto \tau\left(D_{--+}\left(\alpha \beta \alpha^{-1} \beta^{-1}, \beta ; \alpha^{-1}, \alpha^{-1}\right)\right)(c \otimes d)=\varphi_{\alpha^{-1}}(c d) .
$$

Thus, $\operatorname{Tr}\left(\mu_{c} \varphi_{\beta}: L_{\alpha} \rightarrow L_{\alpha}\right)=\tau(P, g)(c)=\operatorname{Tr}\left(\varphi_{\alpha^{-1}} \mu_{c}: L_{\beta} \rightarrow L_{\beta}\right)$.

7.4. Example. By Section 3.3, any $a \in H^{2}\left(G ; K^{*}\right)$ determines a 2-dimensional $X$-HQFT $\left(A^{a}, \tau^{a}\right)$, where $X=K(G, 1)$ with base point $x$. By Section $6.2, a$ determines an isomorphism class of crossed $G$-algebras $L^{a}$.

Theorem 7.2. For any $a \in H^{2}\left(G ; K^{*}\right)$, the underlying crossed $G$-algebra of $\left(A^{a}, \tau^{a}\right)$ is isomorphic to $L^{-a}$.

Proof. Set $S^{1}=\{z \in \mathbb{C}|| z \mid=1\}$ with clockwise orientation and base point $-i$. For each $\alpha \in G=\pi_{1}(X, x)$, fix a loop $u_{\alpha}: S^{1} \rightarrow X$ carrying $-i$ to $x$ and representing $\alpha$. We choose $u_{1}$ to be the constant loop at $x$. Let $p:[0,1] \rightarrow S^{1}$ be the map carrying $t \in[0,1]$ to $-i \exp (-2 \pi i t) \in S^{1}$. Let $\Delta \subset \mathbb{R}^{3}$ be the standard 2-simplex with the vertices $v_{0}=(1,0,0), v_{1}=(0,1,0), v_{2}=(0,0,1)$. For 
$\alpha, \beta \in G$, pick a map $f_{\alpha, \beta}: \Delta \rightarrow X$ such that

$$
\begin{aligned}
& f_{\alpha, \beta}\left((1-t) v_{0}+t v_{1}\right)=u_{\alpha} p(t), \\
& f_{\alpha, \beta}\left((1-t) v_{1}+t v_{2}\right)=u_{\beta} p(t), \\
& f_{\alpha, \beta}\left((1-t) v_{0}+t v_{2}\right)=u_{\alpha \beta} p(t) .
\end{aligned}
$$

for all $t \in[0,1]$. We represent $a \in H^{2}\left(G ; K^{*}\right)=H^{2}\left(X ; K^{*}\right)$ by a $K^{*}$-valued singular 2-cocyle $\Theta$ on $X$. For any $\alpha, \beta \in G$, let $\theta_{\alpha, \beta}=\Theta\left(f_{\alpha, \beta}\right) \in K^{*}$ be the evaluation of $\Theta$ on the singular simplex $f_{\alpha, \beta}$. Observe that $\theta_{\alpha, \beta}$ does not depend on the choice of $f_{\alpha, \beta}$. Indeed, if $f_{\alpha, \beta}^{\prime}: \Delta \rightarrow X$ is another map as above, then the formal difference $f_{\alpha, \beta}-f_{\alpha, \beta}^{\prime}$ is a singular 2-cycle in $X$. The homology class of this 2-cycle is trivial because it can be realized by a map $S^{2} \rightarrow X$ and $\pi_{2}(X)=0$. Therefore $\Theta\left(f_{\alpha, \beta}\right)=\Theta\left(f_{\alpha, \beta}^{\prime}\right)$. A similar argument shows that $\theta=\left\{\theta_{\alpha, \beta}\right\}_{\alpha, \beta \in G}$ is a 2-cocycle. Multiplying, if necessary, $\Theta$ by a coboundary, we can ensure that $\theta_{1,1}=1$. Both $\Theta$ and $\theta$ represent $a \in H^{2}\left(G ; K^{*}\right)$ and $\theta_{-}=\left\{\theta_{\alpha, \beta}^{-1}\right\}_{\alpha, \beta \in G}$ represents $-a$.

Let $L$ be the crossed $G$-algebra underlying the HQFT $\left(A^{\Theta}, \tau^{\Theta}\right)$. We prove that $L$ is isomorphic to the crossed $G$-algebra $L^{\theta_{-}}$determined by the cocycle $\theta_{-}$.

For $\alpha \in G$, the pair $\left(S^{1}, u_{\alpha}\right)$ is an $X$-curve denoted $M_{\alpha}$. The singular 1-simplex $p:[0,1] \rightarrow S^{1}$ is a fundamental cycle of $M_{\alpha}$. By definition of $\left(A^{\Theta}, \tau^{\Theta}\right)$ and $L$, this cycle determines a generating vector $p_{\alpha}=\langle p\rangle$ in $L_{\alpha}=A_{M_{\alpha}}^{\Theta} \cong K$. We claim that $p_{\alpha} p_{\beta}=\theta_{\alpha, \beta}^{-1} p_{\alpha \beta}$ for all $\alpha, \beta \in G$. To compute $p_{\alpha} p_{\beta}$, we apply $\tau^{\Theta}$ to the disk with holes $D=D_{--+}(\alpha, \beta ; 1,1)$ viewed as an $X$-cobordism between $M_{\alpha} \amalg M_{\beta}$ and $M_{\alpha \beta}$. By definition, $p_{\alpha} p_{\beta}=k p_{\alpha \beta}$ for $k=g^{*}(\Theta)(B)$, where $g: D \rightarrow X$ is the map determined by the tuple $(\alpha, \beta ; 1,1)$ and $B \in C_{2}(D ; \mathbb{Z})$ is a fundamental singular 2-chain in $D$ such that $\partial B=p_{\alpha \beta}-p_{\alpha}-p_{\beta}$. Recall the segments $t y$, $t z \subset D$. Clearly, $D^{\prime}=D / t y \cup t z$ is an oriented triangle with oriented edges and identified vertices. Deforming $g$ in its homotopy class, we may assume that $g(t y \cup t z)=x$. Then $g$ expands as the composition of the projection $q: D \rightarrow D^{\prime}$ with a map $g^{\prime}: D^{\prime} \rightarrow X$, and $k$ is the evaluation of $\left(g^{\prime}\right)^{*}(\Theta)$ on $q_{*}(B)$. Instead of $q_{*}(B)$ we can use more general 2-chains in $D^{\prime}$. Namely, let $\partial D^{\prime}=q(\partial D)$ be the union of the sides of $D^{\prime}$. Then $k=\left(g^{\prime}\right)^{*}(\Theta)\left(B^{\prime}\right)$ for any 2-chain $B^{\prime}$ in $D^{\prime}$ such that $\partial B^{\prime}=q p_{\alpha \beta}-q p_{\alpha}-q p_{\beta}$ and the image of $B$ in $C_{2}\left(D^{\prime}, \partial D^{\prime} ; \mathbb{Z}\right)$ represents the generator of $H_{2}\left(D^{\prime}, \partial D^{\prime} ; \mathbb{Z}\right)=\mathbb{Z}$ determined by the orientation of $D^{\prime}$. There is an obvious projection $f: \Delta \rightarrow D^{\prime}$ identifying the vertices of $\Delta$ and such that $g^{\prime} f=f_{\alpha, \beta}$ (up to homotopy relative to $\partial \Delta$ ). The singular chain $B^{\prime}=-f$ satisfies all the conditions above. Therefore

$$
k=\left(g^{\prime}\right)^{*}(\Theta)\left(B^{\prime}\right)=\left(\left(g^{\prime}\right)^{*}(\Theta)(f)\right)^{-1}=\left(\Theta\left(g^{\prime} f\right)\right)^{-1}=\left(\Theta\left(f_{\alpha, \beta}\right)\right)^{-1}=\theta_{\alpha, \beta}^{-1} .
$$

By definition of $L^{\theta_{-}}$, the formula $p_{\alpha} \mapsto \ell_{\alpha}$ for $\alpha \in G$ defines an isomorphism of $G$-algebras $L \cong L^{\theta_{-}}$. It remains to compare the inner products and the actions of $G$. The inner product, $\eta^{-}$, on $L^{\theta-}$ satisfies $\eta^{-}\left(1_{L}, 1_{L}\right)=1$. By Remark 8.2 below, the 
inner product $\eta$ on $L$ satisfies $\eta\left(1_{L}, 1_{L}\right)=\tau^{\Theta}\left(S^{2}\right)=1$. Since $L_{1}=L_{1}^{\theta-}=K$, we have $\eta^{-}=\eta$. The isomorphism $L \cong L^{\theta-}$ commutes with the action of $G$ because there is only one action of $G$ on $L^{\theta_{-}}$satisfying the axioms of a crossed $G$-algebra, cf. Section 6.2.

\section{Properties of the underlying crossed $G$-algebras}

As above, $X=K(G, 1)$ with base point $x$.

8.1. Functoriality. An isomorphism of 2-dimensional $X$-HQFTs induces an isomorphism of the underlying crossed $G$-algebras in the obvious way. This defines a functor from the category of 2-dimensional $X$-HQFTs and their isomorphisms to the category of crossed $G$-algebras and their isomorphisms. This functor is an equivalence of categories, see [12]. We will need only the following weaker claim.

Lemma 8.1. Any isomorphism of the underlying crossed G-algebras of 2-dimensional X-HQFTs is induced by an isomorphism of the HQFTs themselves.

Proof. We first show how to reconstruct a 2-dimensional $X$-HQFT $(A, \tau)$ from its underlying crossed $G$-algebra $L=(L, \eta, \varphi)$. The discussion at the beginning of Section 7.3 shows that $A$ is determined by $L$. We need only to reconstruct $\tau$.

It is clear that every 2-dimensional $X$-cobordism $W$ can be obtained by gluing several $X$-cobordisms whose underlying surfaces are disks with $\leq 2$ holes. Axioms of an HQFT imply that $\tau(W)$ is determined by the values of $\tau$ on such $X$-cobordisms and the restrictions $\left\{\eta_{\alpha}: L_{\alpha} \otimes L_{\alpha^{-1}} \rightarrow K\right\}_{\alpha \in G}$ of $\eta$. It remains to show that the values of $\tau$ on the disks with $\leq 2$ holes are determined by $L$.

We begin by computing $\tau$ for annuli. An $X$-annulus is $X$-homeomorphic to $C_{-+}(\alpha ; \beta), C_{--}(\alpha ; \beta)$, or $C_{++}(\alpha ; \beta)$ with $\alpha, \beta \in G$. By definition, $\tau\left(C_{-+}(\alpha ; \beta)\right)=$ $\left.\varphi_{\beta^{-1}}\right|_{L_{\alpha}}$. The annulus $C_{--}(\alpha ; \beta)$ can be obtained by gluing the annuli $C_{-+}(\alpha ; \beta)$ and $C_{--}\left(\beta^{-1} \alpha \beta ; 1\right)$ along $\left(C_{+}^{1}, \beta^{-1} \alpha \beta\right) \approx\left(C_{-}^{0}, \beta^{-1} \alpha \beta\right)$. Axiom (3) of an HQFT and the definition of $\eta$ imply that $\tau\left(C_{--}(\alpha ; \beta)\right): L_{\alpha} \otimes L_{\beta^{-1} \alpha^{-1} \beta} \rightarrow K$ carries $a \otimes b$ to $\eta\left(\varphi_{\beta^{-1}}(a), b\right)$ for any $a \in L_{\alpha}, b \in L_{\beta^{-1} \alpha^{-1} \beta}$. To compute the vector

$$
\tau\left(C_{++}(\alpha ; \beta)\right) \in \operatorname{Hom}_{K}\left(K, L_{\alpha} \otimes L_{\beta^{-1} \alpha^{-1} \beta}\right)=L_{\alpha} \otimes L_{\beta^{-1} \alpha^{-1} \beta},
$$

we expand it as a finite sum $\sum_{i} p_{i} \otimes q_{i}$, where $p_{i} \in L_{\alpha}$ and $q_{i} \in L_{\beta^{-1} \alpha^{-1} \beta}$. The gluing of $C_{--}\left(\alpha^{-1} ; 1\right)$ to $C_{++}(\alpha ; \beta)$ along $\left(C_{-}^{1}, \alpha\right) \approx\left(C_{+}^{0}, \alpha\right)$ yields $C_{-+}\left(\alpha^{-1} ; \beta\right)$. The axioms of an HQFT yield that $\sum_{i} \eta_{\alpha}\left(a, p_{i}\right) q_{i}=\varphi_{\beta^{-1}}(a)$ for all $a \in L_{\alpha^{-1}}$. Since $\eta_{\alpha}$ is non-degenerate, this uniquely determines $\tau\left(C_{++}(\alpha ; \beta)\right)=\sum_{i} p_{i} \otimes q_{i}$.

There are two $X$-disks: $B_{+}$as in the proof of Lemma 7.1 and $B_{-}$obtained from $B_{+}$by reversing the orientation of the boundary. The vector $\tau\left(B_{+}\right)=1_{L}$ is determined by $L$. The $X$-disk $B_{-}$may be obtained by gluing $B_{+}$and $C_{--}(1 ; 1)$ along $\partial B_{+} \approx C_{-}^{0}$. Therefore $\tau\left(B_{-}\right): L_{1} \rightarrow K$ carries any $a \in L_{1}$ to $\eta\left(1_{L}, a\right)$. 
An $X$-disk with two holes $D$ splits along disjoint loops parallel to its boundary components into three $X$-annuli and a smaller $X$-disk with two holes $D^{\prime}$. Choosing appropriate orientations of the loops and an appropriate map $D \rightarrow X$ in the given homotopy class, we can ensure that $D^{\prime}$ is $X$-homeomorphic to $D_{--+}(\alpha, \beta ; 1,1)$ for some $\alpha, \beta \in G$. The homomorphism $\tau\left(D^{\prime}\right)$ and the values of $\tau$ on the annuli are then determined by $L$. The axioms of an HQFT allow us to recover $\tau(D)$. We conclude that $(A, \tau)$ can be entirely reconstructed from $L$.

We now prove the claim of the theorem. Let $(A, \tau)$ and $\left(A^{\prime}, \tau^{\prime}\right)$ be 2-dimensional $X$-HQFTs with underlying crossed $G$-algebras $L, L^{\prime}$, respectively. An isomorphism $\rho: L \rightarrow L^{\prime}$ defines in the obvious way an isomorphism $A_{M} \rightarrow A_{M}^{\prime}$ for any connected $X$-curve $M$. This extends to non-connected $M$ via $\otimes$. We claim that these isomorphisms $\left\{A_{M} \rightarrow A_{M}^{\prime}\right\}_{M}$ make the natural square diagrams associated with $X$ homeomorphisms and $X$-cobordisms commutative. The part concerning the homeomorphisms is obvious. Each $X$-cobordism can be obtained by gluing $X$-surfaces of type $B_{+}, D_{--+}(\alpha, \beta ; 1,1)$, and annuli. It suffices to check the commutativity of the corresponding diagrams. For $B_{+}$and $D_{--+}(\alpha, \beta ; 1,1)$, the commutativity follows from the assumption that $\rho$ is an algebra isomorphism. For annuli, the commutativity follows from the computations above because $\rho$ preserves the inner product and commutes with the action of $G$.

8.2. Remark. The computation of $\tau\left(B_{ \pm}\right)$in the proof of Lemma 8.1 allows us to compute $\tau\left(S^{2}\right) \in K$ in terms of the underlying crossed $G$-algebra $(L, \eta, \varphi)$ of $(A, \tau)$. The unique homotopy class of maps $S^{2} \rightarrow X$ turns $S^{2}$ into an $X$-manifold. This $X$ manifold can be obtained from $B_{+}$and $B_{-}$by gluing along the boundary. Therefore the homomorphism $K \rightarrow K, k \mapsto \tau\left(S^{2}\right) k$ is the composition of $\tau\left(B_{+}\right): K \rightarrow L_{1}$ with $\tau\left(B_{-}\right): L_{1} \rightarrow K$. Thus, $\tau\left(S^{2}\right)=\eta\left(1_{L}, 1_{L}\right)$.

8.3. Transformations. The following lemmas show that under the passage to the underlying algebra, the direct sums, rescalings, and transfers of HQFTs correspond to the direct sums, rescalings, and transfers of algebras.

Lemma 8.2. If $\left(A^{1}, \tau^{1}\right)$ and $\left(A^{2}, \tau^{2}\right)$ are $X$-HQFTs with underlying crossed $G$-algebras $L^{1}$ and $L^{2}$, respectively, then the underlying crossed $G$-algebra of the direct sum $\left(A^{1}, \tau^{1}\right) \oplus\left(A^{2}, \tau^{2}\right)$ is $L^{1} \oplus L^{2}$. Moreover, if the underlying crossed $G$-algebra $L$ of a 2-dimensional $X$-HQFT $(A, \tau)$ splits as a direct sum of two crossed $G$-algebras $L=L^{1} \oplus L^{2}$, then $(A, \tau)$ splits as a direct sum of two $X$-HQFTs with underlying crossed $G$-algebras $L^{1}$ and $L^{2}$.

Proof. The first claim follows from the definitions. We prove the second claim. The splitting $L_{\alpha}=L_{\alpha}^{1} \oplus L_{\alpha}^{2}$ for all $\alpha \in G$ induces a splitting $A_{M}=A_{M}^{1} \oplus$ $A_{M}^{2}$ for any connected $X$-curve $M$. For a non-connected $X$-curve $M$, we define $A_{M}^{1}, A_{M}^{2}$ via Axiom (4) of an HQFT. This yields a natural embedding $i_{M}^{k}: A_{M}^{k} \rightarrow$ $A_{M}$ and a natural projection $p_{M}^{k}: A_{M} \rightarrow A_{M}^{k}$, where $k=1,2$. Observe now 
that given a connected 2-dimensional $X$-cobordism $(W, M, N)$, the homomorphism $\tau(W): A_{M} \rightarrow A_{N}$ expands uniquely as $i_{N}^{1} \tau^{1}(W) p_{M}^{1}+i_{N}^{2} \tau^{2}(W) p_{M}^{2}$ for some $\tau^{k}(W) \in \operatorname{Hom}\left(A_{M}^{k}, A_{N}^{k}\right)$, where $k=1,2$. For cobordisms of type $D_{--+}, C_{--}$, $C_{-+}$, and $B_{+}$this follows from the splitting $L=L^{1} \oplus L^{2}$. For other cobordisms, this is obtained by splitting them into disks with $\leq 2$ holes as in the proof of Lemma 8.1. It is easy to check that $\left(A^{1}, \tau^{1}\right)$ and $\left(A^{2}, \tau^{2}\right)$ are $X$-HQFTs and $(A, \tau)$ is their direct sum.

Lemma 8.3. If an $\operatorname{HQFT}\left(A^{\prime}, \tau^{\prime}\right)$ is obtained from a 2-dimensional HQFT $(A, \tau)$ by $k$-rescaling with $k \in K^{*}$, then the underlying crossed $G$-algebra of $\left(A^{\prime}, \tau^{\prime}\right)$ is obtained from the underlying crossed $G$-algebra of $(A, \tau)$ by $k$-rescaling.

Proof. For a cobordism $(W, M, N)$ of type $D_{--+}, C_{--}$, and $C_{-+}$, the number $\chi(W)+b_{0}(M)-b_{0}(N)$ is equal to $-1+2-1=0,0+2-1=1$, and $0+1-1=0$, respectively.

Lemma 8.4. Let $H \subset G$ be a subgroup of finite index, $a \in H^{2}\left(H ; K^{*}\right)$, and $k \in K^{*}$. The underlying crossed $G$-algebra of the $X-H Q F T\left(A^{G, H, a}, \tau^{G, H, a, k}\right)$ is isomorphic to the transfer $L^{G, H,-a, k}$ of the crossed $H$-algebra $L^{-a, k}$.

Proof. By the previous lemma, it is enough to consider the case $k=1$. Let $p: \tilde{X} \rightarrow$ $X$ be the covering corresponding to $H \subset G$. Set $Y=\tilde{X} / p^{-1}(x)$ and consider the $Y$ $\operatorname{HQFT}\left(A^{a}, \tau^{a}\right)$ determined by $a \in H^{2}\left(H ; K^{*}\right)=H^{2}\left(\tilde{X} ; K^{*}\right)=H^{2}\left(Y ; K^{*}\right)$. By definition, the HQFT $\left(A^{\prime}=A^{G, H, a}, \tau^{\prime}=\tau^{G, H, a, 1}\right)$ is the transfer of $\left(A^{a}, \tau^{a}\right)$ to $X$. Let $L \cong L^{-a}$ be the underlying crossed $H$-algebra of $\left(A^{a}, \tau^{a}\right)$. We compute the underlying crossed $G$-algebra $\left(L^{\prime}, \eta^{\prime}, \varphi^{\prime}\right)$ of $\left(A^{\prime}, \tau^{\prime}\right)$. Pick a base point $\tilde{x} \in p^{-1}(x)$ of $\tilde{X}$. For each $y \in p^{-1}(x)$ fix a path in $\tilde{X}$ leading from $\tilde{x}$ to $y$. Let $\omega_{y} \in G=\pi_{1}(X, x)$ be the homotopy class of the loop obtained by projecting this path to $X$. Then $\left\{\omega_{y}\right\}_{y \in p^{-1}(x)}$ is a set of representatives of the right $H$-cosets in $G$. For an $X$-curve $M=(M, g: M \rightarrow X)$, the vector space $A_{M}^{\prime}$ is a direct sum of copies of $K$ numerated by the lifts of $g$ to $\tilde{X}$. If $M$ is connected and $g: M \rightarrow X$ represents $\alpha \in G$, then the lifts of $g$ to $\tilde{X}$ are numerated by $y \in p^{-1}(x)$ such that $\omega_{y} \alpha \omega_{y}^{-1} \in H$. Thus, $L_{M}^{\prime}$ is the direct sum of copies of $K$ numerated by such $y \in p^{-1}(x)$. Comparing with the definition of the transfer $(\tilde{L}, \tilde{\eta}, \tilde{\varphi})$ of $L$ (determined by the same set of representatives $\left.\left\{\omega_{y}\right\} y\right)$, we obtain that $L^{\prime}=\tilde{L}$ as $G$-graded vector spaces. Multiplication in $L^{\prime}$ is defined using the maps $D_{--+} \rightarrow X$ carrying the segments $t y, t z \subset D_{--+}$to the base point $x$. A lift of such a map to $\tilde{X}$ carries these segments to the same point of $p^{-1}(x)$ and induces multiplication in the corresponding copy of $K$. Therefore, multiplication in $L^{\prime}$ is a direct sum of multiplications in the copies of $K$ indexed by the same point of $p^{-1}(x)$. Hence $L^{\prime}=\tilde{L}$ as $G$-graded algebras. That $\eta^{\prime}=\tilde{\eta}$ is proven similarly. The definition of the transfer implies that for any $\alpha \in G$, both $\varphi_{\alpha}^{\prime}$ and $\tilde{\varphi}_{\alpha}$ carry $K=L_{\omega_{j} \beta \omega_{j}^{-1}} \subset L_{\beta}^{\prime}=\tilde{L}_{\beta}$ to $K=L_{\omega_{\alpha(j)} \alpha \beta \alpha^{-1} \omega_{\alpha(j)}^{-1}} \subset L_{\alpha \beta \alpha^{-1}}^{\prime}=\tilde{L}_{\alpha \beta \alpha^{-1}}$, where we use the notation of Section 6.3. The equality $\varphi_{\alpha}^{\prime}=\tilde{\varphi}_{\alpha}$ follows then from 
the uniqueness of the action of $G$ on $L$ satisfying the axioms of a crossed $G$-algebra (Section 6.2).

\section{The $G$-center of a biangular $G$-algebra}

Let $B=\bigoplus_{\alpha \in G} B_{\alpha}$ be a biangular $G$-algebra. We compute the crossed $G$-algebra underlying the $\mathrm{HQFT}\left(A_{B}^{\circ}, \tau_{B}^{\circ}\right)$ derived from $B$.

We begin with algebraic preliminaries. For $\alpha \in G$, define a $K$-linear homomorphism $\psi_{\alpha}: B \rightarrow B$ by $\psi_{\alpha}(a)=\sum_{i} p_{i}^{\alpha} a q_{i}^{\alpha}$, where $\sum_{i} p_{i}^{\alpha} \otimes q_{i}^{\alpha}=b_{\alpha} \in B_{\alpha} \otimes B_{\alpha^{-1}}$ is the vector derived from the inner product $\eta=\eta_{B}$ in Section 4.1. Here $i$ runs over a finite set of indices $J_{\alpha}$ and $p_{i}^{\alpha} \in B_{\alpha}, q_{i}^{\alpha} \in B_{\alpha^{-1}}$. Clearly, $\psi_{\alpha}\left(B_{\beta}\right) \subset B_{\alpha \beta \alpha^{-1}}$ for all $\beta \in G$. By (10), $\psi_{\alpha}\left(1_{B}\right)=1_{B}$. The symmetry of $\eta$ implies that

$$
\psi_{\alpha^{-1}}(a)=\sum_{j \in J_{\alpha^{-1}}} p_{j}^{\alpha^{-1}} a q_{j}^{\alpha^{-1}}=\sum_{i \in J_{\alpha}} q_{i}^{\alpha} a p_{i}^{\alpha} .
$$

The following lemma exhibits the main properties of the homomorphisms $\left\{\psi_{\alpha}\right\}_{\alpha}$.

Lemma 9.1. For any $\alpha, \beta \in G$ and $a, b \in B$,

$$
\begin{aligned}
\eta\left(\psi_{\alpha}(a), b\right) & =\eta\left(a, \psi_{\alpha^{-1}}(b)\right), \\
\psi_{\alpha}\left(a \psi_{\beta}(b)\right) & =\psi_{\alpha}(a) \psi_{\alpha \beta}(b), \\
\psi_{\alpha} \psi_{\beta}(b) & =\psi_{\alpha \beta}(b) .
\end{aligned}
$$

If $b \in B_{\beta}$, then for any $\alpha \in G$,

$$
\psi_{\alpha \beta}(b)=\psi_{\alpha}(b)
$$

and for any $\alpha \in G, a \in B$,

$$
\psi_{\alpha}(a) b=b \psi_{\beta^{-1} \alpha}(a) .
$$

For any $\alpha, \beta \in G$ and $c \in B_{\alpha \beta \alpha^{-1} \beta^{-1}}$,

$$
\operatorname{Tr}\left(\mu_{c} \psi_{\beta}: B_{\alpha} \rightarrow B_{\alpha}\right)=\operatorname{Tr}\left(\psi_{\alpha^{-1}} \mu_{c}: B_{\beta} \rightarrow B_{\beta}\right),
$$

where $\mu_{c}$ is the homomorphism $B \rightarrow B, a \mapsto c a$.

Proof. We first check that for any $\beta \in G$ and $b \in B_{\beta^{-1}}$,

$$
\sum_{i \in J_{\alpha}} p_{i}^{\alpha} \otimes q_{i}^{\alpha} b=\sum_{j \in J_{\beta \alpha}} b p_{j}^{\beta \alpha} \otimes q_{j}^{\beta \alpha} .
$$

Both sides belong to $B_{\alpha} \otimes B_{\alpha^{-1} \beta^{-1}}$ and it suffices to prove that they determine equal functionals on the dual space $B_{\alpha^{-1}} \otimes B_{\beta \alpha}$. Pick $x \in B_{\alpha^{-1}}$ and $y \in B_{\beta \alpha}$. By (11), the left-hand side of (27) evaluated on $x \otimes y$ gives

$$
\sum_{i} \eta\left(p_{i}^{\alpha}, x\right) \eta\left(q_{i}^{\alpha} b, y\right)=\sum_{i} \eta\left(x, p_{i}^{\alpha}\right) \eta\left(q_{i}^{\alpha}, b y\right)=\eta(x, b y) .
$$


Note that the expression $\eta(a b, c)$ with $a, b, c \in B$ is invariant under cyclic permutations of $a, b, c$; indeed, $\eta(a b, c)=\eta(a, b c)=\eta(b c, a)$. Therefore the right-hand side of (27) evaluated on $x \otimes y$ gives

$$
\sum_{j} \eta\left(b p_{j}^{\beta \alpha}, x\right) \eta\left(q_{j}^{\beta \alpha}, y\right)=\sum_{j} \eta\left(x b, p_{j}^{\beta \alpha}\right) \eta\left(q_{j}^{\beta \alpha}, y\right)=\eta(x b, y)=\eta(x, b y) .
$$

This proves (27). Formula (27) implies that for any $a \in B, b \in B_{\beta^{-1}}$,

$$
\psi_{\alpha}(a) b=\sum_{i \in J_{\alpha}} p_{i}^{\alpha} a q_{i}^{\alpha} b=\sum_{j \in J_{\beta \alpha}} b p_{j}^{\beta \alpha} a q_{j}^{\beta \alpha}=b \psi_{\beta \alpha}(a) .
$$

This implies (25). By the cyclic symmetry,

$$
\eta\left(\psi_{\alpha}(a), b\right)=\eta\left(\sum_{i} p_{i}^{\alpha} a q_{i}^{\alpha}, b\right)=\eta\left(a, \sum_{i} q_{i}^{\alpha} b p_{i}^{\alpha}\right)=\eta\left(a, \psi_{\alpha^{-1}}(b)\right) .
$$

This proves (21). Using (27), we obtain

$$
\psi_{\alpha}\left(a \psi_{\beta}(b)\right)=\sum_{i \in J_{\alpha}} p_{i}^{\alpha} a \psi_{\beta}(b) q_{i}^{\alpha}=\sum_{i \in J_{\alpha}} p_{i}^{\alpha} a q_{i}^{\alpha} \psi_{\alpha \beta}(b)=\psi_{\alpha}(a) \psi_{\alpha \beta}(b) .
$$

This proves (22). Substituting $a=1_{B}$ in (22), we obtain (23).

Formula (27) implies that for any $b \in B_{\beta^{-1}}$,

$$
\psi_{\alpha^{-1}}(b)=\sum_{i \in J_{\alpha}} q_{i}^{\alpha} b p_{i}^{\alpha}=\sum_{j \in J_{\beta \alpha}} q_{j}^{\beta \alpha} b p_{j}^{\beta \alpha}=\psi_{(\beta \alpha)^{-1}}(b)=\psi_{\alpha^{-1} \beta^{-1}}(b) .
$$

This is equivalent to (24). We now check (26):

$$
\begin{aligned}
\operatorname{Tr}\left(\mu_{c} \psi_{\beta}: B_{\alpha} \rightarrow B_{\alpha}\right) & =\sum_{i \in J_{\alpha}} \eta\left(\mu_{c} \psi_{\beta}\left(p_{i}^{\alpha}\right), q_{i}^{\alpha}\right) \\
& =\sum_{i \in J_{\alpha}, j \in J_{\beta}} \eta\left(c p_{j}^{\beta} p_{i}^{\alpha} q_{j}^{\beta}, q_{i}^{\alpha}\right) \\
& =\sum_{i \in J_{\alpha}, j \in J_{\beta}} \eta\left(q_{i}^{\alpha} c p_{j}^{\beta} p_{i}^{\alpha}, q_{j}^{\beta}\right) \\
& =\sum_{j \in J_{\beta}} \eta\left(\psi_{\alpha^{-1}} \mu_{c}\left(p_{j}^{\beta}\right), q_{j}^{\beta}\right) \\
& =\operatorname{Tr}\left(\psi_{\alpha^{-1}} \mu_{c}: B_{\beta} \rightarrow B_{\beta}\right) .
\end{aligned}
$$

Lemma 9.2. Set $L=\bigoplus_{\alpha \in G} L_{\alpha}$ where $L_{\alpha}=\psi_{1}\left(B_{\alpha}\right) \subset B_{\alpha}$. Then L is a subalgebra of $B$ and $\psi_{\alpha}(L)=L$ for all $\alpha \in G$. The triple $\left(L,\left.\eta\right|_{L},\left\{\left.\psi_{\alpha}\right|_{L}\right\}_{\alpha \in G}\right)$ is a semisimple crossed $G$-algebra.

Proof. Formula (22) with $\alpha=\beta=1$ shows that $L=\operatorname{Im} \psi_{1}$ is a subalgebra of $B$ with unit $1_{B}=\psi_{1}\left(1_{B}\right)$. Formula (23) implies that $\psi_{1}^{2}=\psi_{1}$. By (21), the projector 
$\psi_{1}: B \rightarrow B$ is self-adjoint with respect to $\eta=\eta_{B}$. Hence $B$ splits as an orthogonal sum of $L$ and $\operatorname{Ker} \psi_{1}$. Therefore $\left.\eta\right|_{L}$ is an inner product on $L$.

Formula (23) with $\alpha=1$ shows that $\psi_{\beta}(B) \subset L$ for all $\beta \in G$. Set $\varphi_{\beta}=$ $\left.\psi_{\beta}\right|_{L}: L \rightarrow L$. Formula (23) with $\alpha=\beta^{-1}$ implies that $\varphi_{\beta}$ and $\varphi_{\beta^{-1}}$ are mutually inverse automorphisms of $L$. That they preserve multiplication follows from (22) with $\beta=1$. By (23) and (21), $\varphi_{\alpha \beta}=\varphi_{\alpha} \varphi_{\beta}$ and $\varphi_{\alpha}$ preserves $\left.\eta\right|_{L}$. Formula (24) with $\alpha=1$ yields $\varphi_{\beta} \mid L_{\beta}=$ id. Formula (25) implies that $\varphi_{\alpha}(b) a=a b$ for $a \in L_{\alpha}$, $b \in L$. Finally, for any $\alpha, \beta \in G$ and $c \in L_{\alpha \beta \alpha^{-1} \beta^{-1}}$,

$$
\begin{aligned}
\operatorname{Tr}\left(\mu_{c} \varphi_{\beta}: L_{\alpha} \rightarrow L_{\alpha}\right) & =\operatorname{Tr}\left(\mu_{c} \psi_{\beta}: L_{\alpha} \rightarrow L_{\alpha}\right) \\
& =\operatorname{Tr}\left(\mu_{c} \psi_{\beta}: B_{\alpha} \rightarrow B_{\alpha}\right) \\
& =\operatorname{Tr}\left(\psi_{\alpha^{-1}} \mu_{c}: B_{\beta} \rightarrow B_{\beta}\right) \\
& =\operatorname{Tr}\left(\psi_{\alpha^{-1}} \mu_{c}: L_{\beta} \rightarrow L_{\beta}\right) \\
& =\operatorname{Tr}\left(\varphi_{\alpha^{-1}} \mu_{c}: L_{\beta} \rightarrow L_{\beta}\right),
\end{aligned}
$$

where the second and fourth equalities follow from the inclusion $\psi_{\beta}(B) \subset L$ and the third equality is (26). Thus $L$ satisfies all axioms of a crossed $G$-algebra.

The radical of a finite-dimensional $K$-algebra $C$ can be defined as the annihilator of the symmetric bilinear form $C \times C \rightarrow K$ carrying a pair $\left(c_{1}, c_{2}\right) \in C \times C$ to the trace of the homomorphism $C \rightarrow C, c \mapsto c_{1} c_{2} c$. By the definition of a biangular $G$-algebra, the radical of $B_{1}$ is trivial. Hence $B_{1}$ is a direct sum of matrix rings over $K$. If $B_{1}$ is a matrix ring, $B_{1}=\operatorname{Mat}_{n}(K)$ with $n \geq 1$, then $\eta=\eta_{B}$ carries a pair $\left(a_{1}, a_{2}\right) \in B_{1} \times B_{1}$ to $n \operatorname{Tr}\left(a_{1} a_{2}\right)$, where $\operatorname{Tr}$ is the standard matrix trace on $\operatorname{Mat}_{n}(K)$. The vector $b_{1} \in B_{1} \otimes B_{1}$ determined by $\left.\eta\right|_{B_{1}}$ is equal to $n^{-1} \sum_{i, j=1}^{n} e_{i, j} \otimes e_{j, i}$ where $e_{i, j}$ is the elementary $(n \times n)$-matrix whose $(i, j)$-term is 1 and all other terms are zero. The homomorphism $\psi_{1}: B_{1} \rightarrow B_{1}$ carries any $a \in B_{1}$ to $n^{-1} \sum_{i, j=1}^{n} e_{i, j} a e_{j, i}=n^{-1} \operatorname{Tr}(a) E_{n}$ where $E_{n}$ is the unit $(n \times n)$-matrix. Thus, $\psi_{1}$ is a projection of $\operatorname{Mat}_{n}(K)$ onto its 1-dimensional center. If $B_{1}$ is a direct sum of $N$ matrix rings then $\psi_{1}$ is a projection of $B_{1}$ onto its center $K^{N}$. Hence $L_{1}=\psi_{1}\left(B_{1}\right)=K^{N}$. Thus $L$ is semisimple.

The crossed $G$-algebra $L \subset B$ is called the $G$-center of $B$.

Lemma 9.3. The crossed G-algebra $\left(L^{\prime}=\bigoplus_{\alpha \in G} L_{\alpha}^{\prime}, \eta^{\prime},\left\{\varphi_{\alpha}^{\prime}\right\}_{\alpha}\right)$ underlying the $\operatorname{HQFT}\left(A_{B}^{\circ}, \tau_{B}^{\circ}\right)$ is isomorphic to the $G$-center $L$ of $B$.

Proof. To compute $L_{\alpha}^{\prime}$, we represent $\alpha \in G$ by a loop $g: S^{1} \rightarrow X=K(G, 1)$, where $S^{1}=\{z \in \mathbb{C}|| z \mid=1\}$ with clockwise orientation and base point $s=1$. The $X$-curve $M=\left(S^{1}, g\right)$ has a canonical framing $t$ formed by one vertex $s$, one edge $e$, and the $G$-system assigning $\alpha$ to the edge $e$ oriented clockwise. By Section 4.5, $L_{\alpha}^{\prime}=A_{M}^{\circ}$ is the image of the projector $P_{M}: A_{(M, t)} \rightarrow A_{(M, t)}$, where $A_{(M, t)}=B_{\alpha}$. Recall that $P_{M}=\tau\left(W, g_{1}: W \rightarrow X\right)$, where $W=S^{1} \times[0,1]$ is viewed as a cobordism between two copies of $(M, t)$ and $g_{1}$ is the composition of the projection 
$W \rightarrow S^{1}$ with $g: S^{1} \rightarrow X$. Let $T$ be the CW-decomposition of $W$ formed by the vertices $(s, 0),(s, 1)$, the edges $e_{0}=e \times\{0\}, e_{1}=e \times\{1\}, e_{2}=\{s\} \times[0,1]$, and the face $\left(S^{1}-\{s\}\right) \times(0,1)$. We orient $e_{0}, e_{1}$ clockwise and $e_{2}$ from $(s, 0)$ to $(s, 1)$. The map $g_{1}$ is presented by the $G$-system on $T$ assigning $\alpha$ to $e_{0}, e_{1}$ and $1 \in G$ to $e_{2}$. It is clear from the definitions that $P_{M}=\tau\left(W, g_{1}\right): B_{\alpha} \rightarrow B_{\alpha}$ carries any $a \in B_{\alpha}$ to $\sum_{i} p_{i}^{1} a q_{i}^{1}$, where $\sum_{i} p_{i}^{1} \otimes q_{i}^{1}=b_{1} \in B_{1} \otimes B_{1}$. Hence $P_{M}=\psi_{1}$ and $L_{\alpha}^{\prime}=\operatorname{Im} \psi_{1}=L_{\alpha}$.

Replacing in this construction the labels of $e_{1}, e_{2}$ by $\beta \alpha \beta^{-1}, \beta^{-1} \in G$, respectively, we obtain a $G$-system on $T$ representing a map $g_{\beta}: W \rightarrow X$. The $X$-cobordism $\left(W, g_{\beta}\right)$ is the annulus $C_{-+}\left(\alpha ; \beta^{-1}\right)$ used to define $\varphi_{\beta}^{\prime}$ on $L_{\alpha}^{\prime}=L_{\alpha}$ in Section 7.3. By definition,

$$
\left.\varphi_{\beta}^{\prime}\right|_{L_{\alpha}}=\tau^{\circ}\left(W, g_{\beta}\right)=\left.\tau\left(W, g_{\beta}\right)\right|_{L_{\alpha}}: L_{\alpha} \rightarrow L_{\beta \alpha \beta^{-1}} .
$$

The homomorphism $\tau\left(W, g_{\beta}\right): B_{\alpha} \rightarrow B_{\beta \alpha \beta^{-1}}$ carries any $a \in B_{\alpha}$ to $\sum_{i} p_{i}^{\beta} a q_{i}^{\beta}=$ $\psi_{\beta}(a)$, where $\sum_{i} p_{i}^{\beta} \otimes q_{i}^{\beta}=b_{\beta} \in B_{\beta} \otimes B_{\beta^{-1}}$. Hence $\varphi_{\beta}^{\prime}=\left.\psi_{\beta}\right|_{L}$.

Inverting the orientation of $S^{1} \times\{1\} \subset \partial W$, we can view $\left(W, g_{1}\right)$ as an $X$-cobordism between $(M, t) \amalg(-M, t)$ and $\emptyset$. In the notation of Section 7.3, this cobordism is $C_{--}(\alpha ; 1)$. By definition, $\eta_{\alpha}^{\prime}=\tau^{\circ}\left(W, g_{1}\right): L_{\alpha} \otimes L_{\alpha^{-1}} \rightarrow K$ is the restriction of $\tau\left(W, g_{1}\right): B_{\alpha} \otimes B_{\alpha^{-1}} \rightarrow K$. For $a \in B_{\alpha}, b \in B_{\alpha^{-1}}$,

$$
\tau\left(W, g_{1}\right)(a \otimes b)=\sum_{i} \eta_{B}\left(a p_{i}^{1} b q_{i}^{1}, 1_{B}\right)=\eta_{B}\left(a, \psi_{1}(b)\right) .
$$

Since $\left.\psi_{1}\right|_{L}=$ id, we conclude that $\eta^{\prime}=\left.\eta_{B}\right|_{L \otimes L}$.

By the proof of Lemma 9.2, the $K$-algebra $B_{1}$ splits as a direct sum of matrix algebras over $K$ and $\psi_{1}: B_{1} \rightarrow B_{1}$ maps each of these matrix algebras onto its center. The set $I=\operatorname{bas}(L)$ of the basic idempotents of $L_{1}=\psi_{1}\left(B_{1}\right)$ is the set of unit elements of these matrix algebras. More precisely, each basic idempotent $i \in I$ is the unit element of a direct summand $\operatorname{Mat}_{n_{i}}(K)$ of $B_{1}$, where $n_{i} \geq 1$. Let $I_{0} \subset I$ be the fixed point set of the action of $G$ on $I$. For $i \in I_{0}$, the subalgebra $i L$ of $L$ is a crossed $G$-algebra with unique basic idempotent $i$. The pair $(i L, i)$ determines a cohomology class $\nabla_{i} \in H^{2}\left(G ; K^{*}\right)$ by Lemma 6.2.

Theorem 9.4. For any closed connected oriented surface $W$ endowed with a map $\tilde{g}: W \rightarrow X=K(G, 1)$ such that $g=\tilde{g}_{\#}: \pi_{1}(W) \rightarrow G$ is an epimorphism,

$$
\left.\tau_{B}(W, \tilde{g})=\sum_{i \in I_{0}} n_{i}^{\chi(W)} g^{*}\left(\nabla_{i}\right)([-W])\right) .
$$

Proof. By the previous lemma, $L$ is the underlying crossed $G$-algebra of the HQFT $\left(A_{B}^{\circ}, \tau_{B}^{\circ}\right)$. As we know, $L$ splits as a direct sum of simple crossed $G$-algebras $L^{\kappa}$ numerated by the orbits $\kappa$ of the action of $G$ on $I=\operatorname{bas}(L)$. By Lemma 8.2, the HQFT $\left(A_{B}^{\circ}, \tau_{B}^{\circ}\right)$ splits as a direct sum of HQFTs $\left(A^{\kappa}, \tau^{\kappa}\right)$ such that $L^{\kappa}$ is the 
underlying crossed $G$-algebra of $\left(A^{\kappa}, \tau^{\kappa}\right)$ for all orbits $\kappa \subset I$. Then $\tau_{B}(W, \tilde{g})=$ $\tau_{B}^{\circ}(W, \tilde{g})=\sum_{\kappa} \tau^{\kappa}(W, \tilde{g})$.

We claim that $\tau^{\kappa}(W, \tilde{g})=0$ for any orbit $\kappa \subset I$ with at least two elements. Indeed, the proof of Lemma 6.2 shows that $L^{\kappa}$ is isomorphic to $L^{G, H, \theta, k}$, where $H$ is a subgroup of $G$ of index $|m| \geq 2, \theta \in H^{2}\left(H ; K^{*}\right)$, and $k \in K^{*}$. By Lemma 8.4, $L^{G, H, \theta, k}$ underlies the $X$-HQFT $\left(A^{G, H,-\theta}, \tau^{G, H,-\theta, k}\right)$. By Lemma 8.1, this $X$ HQFT is isomorphic to $\left(A^{\kappa}, \tau^{\kappa}\right)$. Thus, $\left(A^{\kappa}, \tau^{\kappa}\right)$ is isomorphic to the transfer of a $K(H, 1)$-HQFT. The assumption $g\left(\pi_{1}(W)\right)=G$ implies that the map $\tilde{g}: W \rightarrow X$ does not lift to non-trivial coverings of $X$. Hence, $\tau^{\kappa}(W, \tilde{g})=0$.

A one-element orbit $\kappa \subset I$ is just $\{i\}$ for $i \in I_{0}$. By Lemma 6.2, $L^{\kappa}=i L$ is obtained from $L^{\nabla_{i}}$ by $k_{i}$-rescaling, where $k_{i}=\eta_{B}(i, i)=n_{i}^{2}$. Hence $L^{\kappa}$ underlies the $X$-HQFT obtained from $\left(A^{-\nabla_{i}}, \tau^{\left.-\nabla_{i}\right)}\right.$ by $k_{i}$-rescaling. By Lemma 8.1, the latter $\mathrm{HQFT}$ is isomorphic to $\left(A^{\kappa}, \tau^{\kappa}\right)$. We have therefore

$$
\tau^{\kappa}(W, \tilde{g})=\tau^{\{i\}}(W, \tilde{g})=k_{i}^{\chi(W) / 2} g^{*}\left(-\nabla_{i}\right)([W])=n_{i}^{\chi(W)} g^{*}\left(\nabla_{i}\right)([-W]) .
$$

Hence

$$
\left.\tau_{B}(W, \tilde{g})=\sum_{i \in I_{0}} \tau^{\{i\}}(W, \tilde{g})=\sum_{i \in I_{0}} n_{i}^{\chi(W)} g^{*}\left(\nabla_{i}\right)([-W])\right) .
$$

Note that for $W=S^{2}$, we have $G=\{1\}$ and $\nabla_{i}=0$ for all $i \in I_{0}=I$. Formula (29) gives in this case $\tau_{B}(W, \tilde{g})=\sum_{i \in I} n_{i}^{2}=\operatorname{dim} B_{1}$.

We now establish an analogue of formula (29) for a compact connected oriented surface $W$ of positive genus whose boundary has $m \geq 1$ components. We use the notation introduced in the first two paragraphs of Section 5.1 assuming that the given homomorphism $g: \pi=\pi_{1}(W, w) \rightarrow G$ is surjective and that its restriction to the free group $H=\left\langle x_{1}, \ldots, x_{m}\right\rangle \subset \pi$ is injective. As above, $L$ is the $G$-center of $B$ and $I_{0}$ is the fixed point set of the action of $G$ on bas $(L)$. Suppose that for each $k=1, \ldots, m$, we are given a vector $\bar{\gamma}_{k} \in L_{g\left(x_{k}\right)}=A_{\left(C_{k}, \tilde{g}\right)}^{\circ}$ where $A^{\circ}=A_{B}^{\circ}$ and $C_{k}$ is the $k$-th component of $\partial W$. Let $I_{0}^{\gamma} \subset I_{0}$ consist of all $i \in I_{0}$ such that $i \bar{\gamma}_{k} \neq 0$ for all $k=1, \ldots, m$ (note that $i \bar{\gamma}_{k}$ is the projection of $\bar{\gamma}_{k}$ to the direct summand $i L_{g\left(x_{k}\right)}$ of $\left.L_{g\left(x_{k}\right)}\right)$. For each $i \in I_{0}^{\gamma}$, we define a cohomology class $\nabla_{i}^{\gamma} \in H^{2}\left(G, g(H) ; K^{*}\right)$ as $\nabla_{i}$ in Lemma 6.2 choosing the generating vectors $s_{\alpha} \in i L_{\alpha} \cong K$ as follows. Set $s_{g\left(x_{k}\right)}=i \bar{\gamma}_{k}$ for all $k$. The properties of the crossed $G$-algebra $i L$ imply that there is a unique vector $s_{g\left(x_{k}\right)^{-1}} \in i L_{g\left(x_{k}\right)^{-1}}$ whose left and right products with $s_{g\left(x_{k}\right)}$ are equal to $i \in i L_{1}$. Each $\alpha \in g(H)$ expands as a product of the generators $g\left(x_{k}\right)^{ \pm 1} \in g(H)$, and we let $s_{\alpha}$ be the product of the corresponding vectors $s_{g\left(x_{k}\right)^{ \pm 1}}$ (note that $s_{\alpha} \neq 0$ ). For $\alpha \in G-g(H)$, the generating vector $s_{\alpha} \in i L_{\alpha}$ is chosen in an arbitrary way. The equality $s_{\alpha} s_{\beta}=\nabla_{\alpha, \beta} s_{\alpha \beta}$ for $\alpha, \beta \in G$ defines a $K^{*}$-valued 2-cocycle $\nabla=\left\{\nabla_{\alpha, \beta}\right\}_{\alpha, \beta \in G}$ whose restriction to $g(H)$ is trivial. This cocycle represents $\nabla_{i}^{\gamma} \in H^{2}\left(G, g(H) ; K^{*}\right)$. 
Theorem 9.5. Set $\bar{\gamma}=\bigotimes_{k=1}^{m} \bar{\gamma}_{k} \in A_{(\partial W, \tilde{g})}^{\circ}$. Then

$$
\left.\tau_{B}^{\circ}(-W, \tilde{g})(\bar{\gamma})=\sum_{i \in I_{0}^{\gamma}} n_{i}^{\chi(W)+m} g^{*}\left(\nabla_{i}^{\gamma}\right)([W, \partial W])\right),
$$

where we view $[W, \partial W]$ as an element of $H_{2}(\pi, H ; \mathbb{Z})$, cf. Lemma 5.2.

Proof. The first part of the proof of Theorem 9.4 applies directly and gives

$$
\tau_{B}(-W, \tilde{g})(\bar{\gamma})=\sum_{i \in I_{0}} \tau^{\{i\}}(-W, \tilde{g})\left(\bigotimes_{k=1}^{m} i \bar{\gamma}_{k}\right)
$$

For $i \in I_{0}-I_{0}^{\gamma}$, the $i$-th term on the right-hand side is equal to 0 . It remains to show that for every $i \in I_{0}^{\gamma}$,

$$
\left.\tau^{\{i\}}(-W, \tilde{g})\left(\bigotimes_{k=1}^{m} i \bar{\gamma}_{k}\right)=n_{i}^{\chi(W)+m} g^{*}\left(\nabla_{i}^{\gamma}\right)([W, \partial W])\right) .
$$

We can assume that $X=K(G, 1)$ contains a CW-subspace $Y=K(g(H), 1)$ with the same base point $x$. Let $p:[0,1] \rightarrow S^{1},\left\{u_{\alpha}: S^{1} \rightarrow X\right\}_{\alpha \in G}, \Delta \subset \mathbb{R}^{3}$, and $\left\{f_{\alpha, \beta}: \Delta \rightarrow X\right\}_{\alpha, \beta \in G}$ be as in the proof of Theorem 7.2. We can assume that $u_{\alpha}\left(S^{1}\right) \subset Y$ for all $\alpha \in g(H)$ and $f_{\alpha, \beta}(\Delta) \subset Y$ for all $\alpha, \beta \in g(H)$. Pick a singular $K^{*}$-valued 2-cocycle $\Theta$ on $X$ such that $\Theta\left(f_{\alpha, \beta}\right)=\nabla_{\alpha, \beta}^{-1}$ for all $\alpha, \beta \in G$, where $\nabla=\left\{\nabla_{\alpha, \beta}\right\}$ is the 2-cocycle on $G$ determined by the vectors $s_{\alpha} \in i L_{\alpha}$ chosen above. Modifying if necessary $\Theta$ by a coboundary, we can assume additionally that $\Theta$ annihilates all singular chains in $Y$ and defines thus a cohomology class $[\Theta] \epsilon$ $H^{2}\left(X, Y ; K^{*}\right)$. This class is equal to $-\nabla_{i}^{\gamma}$ under the identification $H^{2}\left(X, Y ; K^{*}\right)=$ $H^{2}\left(G, g(H) ; K^{*}\right)$. Consider the $X$-HQFT $\left(A^{\Theta}, \tau^{\Theta}\right)$ and its $k_{i}$-rescaling $\left(A^{\Theta}, \tau^{\Theta, k_{i}}\right)$ where $k_{i}=n_{i}^{2}$. Let $R$ and $R^{\prime}$ be the crossed $G$-algebras underlying these two $X$-HQFTs, respectively. For $\alpha \in G$, consider the generating vector

$$
p_{\alpha}=\langle p\rangle \in R_{\alpha}=R_{\alpha}^{\prime}=A_{S^{1}, \alpha}^{\Theta} \cong K
$$

represented by the singular 1 -simplex $p:[0,1] \rightarrow S^{1}$ viewed as a fundamental cycle of the $X$-curve $\left(S^{1}, u_{\alpha}\right)$. By the proof of Theorem 7.2, $p_{\alpha} p_{\beta}=\nabla_{\alpha, \beta} p_{\alpha \beta}$ for all $\alpha, \beta \in G$. Moreover, the formula $s_{\alpha} \mapsto p_{\alpha}$ defines an isomorphism of crossed $G$-algebras $i L \rightarrow R^{\prime}$. By Lemma 8.1, this isomorphism lifts to an isomorphism of $X$-HQFTs $\left(A^{\{i\}}, \tau^{\{i\}}\right) \rightarrow\left(A^{\Theta}, \tau^{\Theta, k_{i}}\right)$. Therefore

$$
\begin{aligned}
\tau^{\{i\}}(-W, \tilde{g})\left(\bigotimes_{k=1}^{m} i \bar{\gamma}_{k}\right) & =\tau^{\{i\}}(-W, \tilde{g})\left(\bigotimes_{k=1}^{m} s_{g\left(x_{k}\right)}\right) \\
& =\tau^{\Theta, k_{i}}(-W, \tilde{g})\left(\bigotimes_{k=1}^{m} p_{g\left(x_{k}\right)}\right) \\
& =n_{i}^{\chi(W)+m} \tau^{\Theta}(-W, \tilde{g})\left(\bigotimes_{k=1}^{m} p_{g\left(x_{k}\right)}\right) .
\end{aligned}
$$

We can choose $\tilde{g}: W \rightarrow X$ in its homotopy class so that the restriction of $\tilde{g}$ to the $k$-th component $C_{k}$ of $\partial W$ is the composition of an orientation-preserving pointed 
homeomorphism $f_{k}: C_{k} \rightarrow S^{1}$ with $u_{g\left(x_{k}\right)}: S^{1} \rightarrow X$ for all $k=1, \ldots, m$. It then follows that $p_{g\left(x_{k}\right)}=\left\langle f_{k}^{-1} p\right\rangle \in A_{\left(C_{k}, \tilde{g}\right)}^{\Theta}$ is represented by the singular 1-simplex $f_{k}^{-1} p:[0,1] \rightarrow C_{k}$ viewed as a fundamental cycle in $C_{k}$. By the definition of $\tau^{\Theta}$,

$$
\left.\tau^{\Theta}(-W, \tilde{g})\left(\bigotimes_{k=1}^{m}\left\langle f_{k}^{-1} p\right\rangle\right)=\tilde{g}^{*}([\Theta])(-[W, \partial W])=g^{*}\left(\nabla_{i}^{\gamma}\right)([W, \partial W])\right) .
$$

Combining these formulas we obtain (32).

\section{Proof of Lemma 5.2}

10.1. Preliminaries. Recall the notation of Lemma 5.2: $q: G^{\prime} \rightarrow G$ is a group epimorphism with finite kernel $\Gamma$ and $B=K\left[G^{\prime}\right]$ is the associated biangular $G$-algebra. By Lemma 9.2, the $G$-center $\left(L=\psi_{1}(B),\left.\eta_{B}\right|_{L},\left\{\left.\psi_{\alpha}\right|_{L}\right\}_{\alpha \in G}\right)$ of $B$ is a semisimple crossed $G$-algebra. Let $I=\operatorname{bas}(L)$ be the $G$-set of its basic idempotents. As we know, each $i \in I$ is the unit element of a direct summand $\operatorname{Mat}_{n_{i}}(K)$ of $B_{1}$, where $n_{i} \geq 1$. Let $\rho_{i}^{+}: B_{1} \rightarrow \operatorname{Mat}_{n_{i}}(K)$ be the projection onto this summand. Clearly, $\rho_{i}^{+}(i)=E_{n_{i}}$, where $E_{n}$ is the unit $n \times n$ matrix. The homomorphism $\rho_{i}^{+}$determines a representation $\rho_{i}=\left.\rho_{i}^{+}\right|_{\Gamma}: \Gamma \rightarrow \mathrm{GL}_{n_{i}}(K)$ of $\Gamma$ and is recovered from $\rho_{i}$ as its linear extension. We can describe $\rho_{i}$ as the unique (up to equivalence) irreducible representation of $\Gamma$ such that $\rho_{i}^{+}(i) \neq 0$. It is clear that the mapping $I \rightarrow \operatorname{Irr}(\Gamma), i \mapsto \rho_{i}$, is a bijection. We show that it is $G$-equivariant. By definition of the action of $G$ on $I$, for $\alpha \in G$ and $i \in I$,

$$
\alpha i=\psi_{\alpha}(i)=|\Gamma|^{-1} \sum_{a \in q^{-1}(\alpha)} a i a^{-1} .
$$

All the summands on the right-hand side are equal because $i$ lies in the center of $L_{1}$. Therefore $\alpha i=a i a^{-1}$ for any $a \in q^{-1}(\alpha)$. By Section 2.1, $a \rho_{i}(b)=\rho_{i}\left(a^{-1} b a\right)$ for all $b \in B_{1}$. Hence $a \rho_{i}(\alpha i)=\rho_{i}(i) \neq 0$. Therefore $a \rho_{i}=\rho_{\alpha i}$ for all $a \in q^{-1}(\alpha)$, i.e., the bijection $I \rightarrow \operatorname{Irr}(\Gamma), i \mapsto \rho_{i}$ is $G$-equivariant. We identify $I$ with $\operatorname{Irr}(\Gamma)$ along this bijection. Though we shall not need it, note that each idempotent $i \in I$ can be computed from the character $\chi_{i}$ of $\rho_{i}$ by $i=|\Gamma|^{-1} \chi_{i}(1) \sum_{h \in \Gamma} \chi_{i}(h) h^{-1}$; see, for instance, [3], Chapter 2, Theorem 5.

In the sequel, $I_{0}=I_{0}(q)$ is the fixed point set of the action of $G$ on $I=\operatorname{Irr}(\Gamma)$ and $\left(A^{\circ}=A_{B}^{\circ}, \tau^{\circ}=\tau_{B}^{\circ}\right)$ is the 2-dimensional HQFT with target $X=K(G, 1)$ determined by $B$.

10.2. The case $\partial W=\emptyset$. For a closed connected oriented surface $W$, Lemma 5.2 is equivalent to the following claim: for any pointed map $\tilde{g}: W \rightarrow X$ inducing an epimorphism $g: \pi_{1}(W) \rightarrow G$,

$$
\tau^{\circ}(W, \tilde{g})=\sum_{i \in I_{0}} n_{i}^{\chi(W)} g^{*}\left(\zeta^{\rho_{i}}\right)([-W]) .
$$


To deduce this formula from (29) it is enough to show that $\nabla_{i}=\zeta^{\rho_{i}}$ for all $i \in I_{0}$. Pick $i \in I_{0}$ and set $\rho=\rho_{i}$ and $n=n_{i}=\operatorname{dim} \rho$. Fix the lifts $\left\{\tilde{\alpha} \in q^{-1}(\alpha)\right\}_{\alpha \in G}$ and the matrices $\left\{M_{\alpha}\right\}_{\alpha} \in \mathrm{GL}_{n}(K)$ as in Section 2.2. This data determines a $K^{*}$-valued 2-cocycle $\left\{\zeta_{\alpha, \beta}\right\}_{\alpha, \beta}$ representing $\zeta^{\rho}$. The proof of Lemma 2.1 yields an extension of $\rho: \Gamma \rightarrow \mathrm{GL}_{n}(K)$ to a map $\bar{\rho}: G^{\prime} \rightarrow \mathrm{GL}_{n}(K)$. Let $\bar{\rho}^{+}: B=K\left[G^{\prime}\right] \rightarrow \operatorname{Mat}_{n}(K)$ be the induced $K$-linear map. Then $\left.\bar{\rho}^{+}\right|_{B_{1}}=\rho^{+}$and in particular $\bar{\rho}^{+}(i)=\rho^{+}(i)=$ $E_{n}$. Since $\bar{\rho}^{+}\left(B_{1}\right)=\operatorname{Mat}_{n}(K)$, for each $\alpha \in G$, there is $d_{\alpha} \in B_{1}$ such that $\bar{\rho}^{+}\left(d_{\alpha}\right)=M_{\alpha}^{-1}=\left(\bar{\rho}^{+}(\tilde{\alpha})\right)^{-1}$. Set

$$
s_{\alpha}=i \psi_{1}\left(d_{\alpha} \tilde{\alpha}\right)=|\Gamma|^{-1} \sum_{a \in \Gamma} i a d_{\alpha} \tilde{\alpha} a^{-1} \in i L_{\alpha} \subset B_{\alpha} .
$$

By (6) and the identity $\zeta_{\alpha, 1}=\zeta_{1, \alpha}=1$, we have $\bar{\rho}^{+}(a b)=\bar{\rho}^{+}(a) \bar{\rho}^{+}(b)$ for $a, b \in B$ provided $a \in B_{1}$ or $b \in B_{1}$. Therefore

$$
\begin{aligned}
\bar{\rho}^{+}\left(s_{\alpha}\right) & =|\Gamma|^{-1} \sum_{a \in \Gamma} \bar{\rho}^{+}\left(\text {iad }_{\alpha} \tilde{\alpha} a^{-1}\right) \\
& =|\Gamma|^{-1} \sum_{a \in \Gamma} \bar{\rho}^{+}(i) \bar{\rho}^{+}(a) \bar{\rho}^{+}\left(d_{\alpha}\right) \bar{\rho}^{+}(\tilde{\alpha}) \bar{\rho}^{+}\left(a^{-1}\right) \\
& =|\Gamma|^{-1} \sum_{a \in \Gamma} \bar{\rho}^{+}(a) \bar{\rho}^{+}\left(a^{-1}\right) \\
& =|\Gamma|^{-1} \sum_{a \in \Gamma} \rho(a) \rho\left(a^{-1}\right)=E_{n} .
\end{aligned}
$$

Thus, $s_{\alpha}$ is a non-zero vector in $i L_{\alpha} \cong K$. We take $\tilde{1}=1 \in G^{\prime}$ and $d_{1}=1 \in B_{1}$ so that $s_{1}=i$. The cohomology class $\nabla_{i}$ is represented by the normalized $K^{*}$-valued 2-cocycle $\left\{\nabla_{\alpha, \beta}\right\}_{\alpha, \beta \in G}$ defined from $s_{\alpha} s_{\beta}=\nabla_{\alpha, \beta} s_{\alpha \beta}$. Formula (6) implies that

$$
\nabla_{\alpha, \beta} E_{n}=\bar{\rho}^{+}\left(\nabla_{\alpha, \beta} s_{\alpha \beta}\right)=\bar{\rho}^{+}\left(s_{\alpha} s_{\beta}\right)=\zeta_{\alpha, \beta} \bar{\rho}^{+}\left(s_{\alpha}\right) \bar{\rho}^{+}\left(s_{\beta}\right)=\zeta_{\alpha, \beta} E_{n} .
$$

Hence, $\nabla_{\alpha, \beta}=\zeta_{\alpha, \beta}$ for all $\alpha, \beta \in G_{\rho}$.

10.3. The case $\partial W \neq \emptyset$. Pick $k \in\{1, \ldots, m\}$. Then $\gamma_{k} \in q^{-1}\left(g\left(x_{k}\right)\right) \subset G^{\prime}$ is one of the generating vectors of $B_{g\left(x_{k}\right)}$ and $\psi_{1}\left(\gamma_{k}\right)$ is its projection to $L_{g\left(x_{k}\right)}$. Set

$$
\bar{\gamma}_{k}=\sum_{i \in I_{0}} i n_{i} \psi_{1}\left(\gamma_{k}\right) \in L_{g\left(x_{k}\right)}
$$

We verify first that $i \bar{\gamma}_{k}=0$ if and only if $t_{\rho_{i}}\left(\gamma_{k}\right)=0$. Fix $i \in I_{0}$ and set $\rho=\rho_{i}$ and $n=n_{i}=\operatorname{dim} \rho$. Fix the lifts $\left\{\tilde{\alpha} \in q^{-1}(\alpha)\right\}_{\alpha \in G}$ and the matrices $\left\{M_{\alpha}\right\}_{\alpha} \in \operatorname{GL}_{n}(K)$ as in Section 2.3. In particular, for $\alpha=g\left(x_{k}\right)$ with $k=1, \ldots, m$, we have $\tilde{\alpha}=\gamma_{k}$ and $\operatorname{Tr} M_{\alpha}=t_{\rho}\left(\gamma_{k}\right) \in\{0,1\}$. We extend $\rho: \Gamma \rightarrow \mathrm{GL}_{n}(K)$ to $\bar{\rho}^{+}: B=K\left[G^{\prime}\right] \rightarrow$ $\operatorname{Mat}_{n}(K)$ as above. Observe that $i \bar{\gamma}_{k}=i n \psi_{1}\left(\gamma_{k}\right) \in i L_{g\left(x_{k}\right)}$. A computation similar to the one in Section 10.2 shows that

$$
\operatorname{Tr} \bar{\rho}^{+}\left(i \bar{\gamma}_{k}\right)=n \operatorname{Tr} \bar{\rho}^{+}\left(i \psi_{1}\left(\gamma_{k}\right)\right)=n \operatorname{Tr} \bar{\rho}^{+}\left(\gamma_{k}\right)=n \operatorname{Tr} M_{g\left(x_{k}\right)}=n t_{\rho}\left(\gamma_{k}\right) .
$$


Consider the generating vectors $\left\{s_{\alpha} \in i L_{\alpha} \cong K\right\}_{\alpha \in G}$ defined in Section 10.2. Comparing the traces of $\bar{\rho}^{+}\left(i \bar{\gamma}_{k}\right)$ and $\bar{\rho}^{+}\left(s_{g\left(x_{k}\right)}\right)=E_{n}$, we obtain that either $i \bar{\gamma}_{k}=0$ and $t_{\rho}\left(\gamma_{k}\right)=0$ or $i \bar{\gamma}_{k}=s_{g\left(x_{k}\right)}$ and $t_{\rho}\left(\gamma_{k}\right)=1$.

If $i \bar{\gamma}_{k} \neq 0$ for all $k=1, \ldots, m$, then the vectors $\left\{s_{\alpha}\right\}_{\alpha \in G}$ satisfy the requirements needed in the definition $\nabla_{i}^{\gamma}$ given before Theorem 9.5. Indeed, the computation at the end of Section 10.2 shows that the 2-cocycle $\left\{\nabla_{\alpha, \beta}\right\}_{\alpha, \beta \in G}$ defined from $s_{\alpha} s_{\beta}=\nabla_{\alpha, \beta} s_{\alpha \beta}$ is equal to the 2-cocycle $\left\{\zeta_{\alpha, \beta}\right\}_{\alpha, \beta \in G}$ derived in Section 2.3 from the lifts $\{\tilde{\alpha}\}_{\alpha \in G}$. In particular, $\nabla_{\alpha, \beta}=\zeta_{\alpha, \beta}=1$ for all $\alpha, \beta \in g(H)$. Therefore $s_{\alpha} s_{\beta}=s_{\alpha \beta}$ for all $\alpha, \beta \in g(H)$. This and the equality $s_{g\left(x_{k}\right)}=i \bar{\gamma}_{k}$ for all $k$ are precisely the conditions used in the definition of $\nabla_{i}^{\gamma}$. We conclude that $\nabla_{i}^{\gamma}=\zeta^{\rho_{i}, \gamma} \in H^{2}\left(G, g(H) ; K^{*}\right)$ and rewrite formula (32) as

$$
\left.\tau^{\{i\}}(-W, \tilde{g})\left(\bigotimes_{k=1}^{m} i \bar{\gamma}_{k}\right)=n_{i}^{\chi(W)+m} g^{*}\left(\zeta^{\rho_{i}, \gamma}\right)([W, \partial W])\right) .
$$

Dividing both sides by $n_{i}^{m}$, we obtain that

$$
\left.\tau^{\{i\}}(-W, \tilde{g})\left(\bigotimes_{k=1}^{m} i \psi_{1}\left(\gamma_{k}\right)\right)=n_{i}^{\chi(W)} g^{*}\left(\zeta^{\rho_{i}, \gamma}\right)([W, \partial W])\right) .
$$

Summing up over all $i \in I_{0}$ such that $i \bar{\gamma}_{k} \neq 0$ for all $k$ and using (31) (with $\bar{\gamma}_{k}$ replaced by $\left.\psi_{1}\left(\gamma_{k}\right)\right)$, we obtain

$$
\left.\tau_{B}(-W, \tilde{g})([\gamma])=\sum_{\substack{i \in I_{0} \\ t_{\rho_{i}}\left(\gamma_{k}\right)=1 \text { for all } k}} n_{i}^{\chi(W)} g^{*}\left(\zeta^{\rho_{i}, \gamma}\right)([W, \partial W])\right) .
$$

This formula is equivalent to the one claimed by the lemma.

\section{The case of a trivial bundle}

For trivial bundles, Theorem 1.2 simplifies but remains non-trivial. We begin with a computation of the cohomology classes $\zeta^{\rho, \gamma}$ for direct products.

Lemma 11.1. Let $G$, $\Gamma$ be groups, $G^{\prime}=G \times \Gamma$ and $q: G^{\prime} \rightarrow G$ be the projection.

(a) The action of $G$ on $\operatorname{Irr}(\Gamma)$ determined by $q$ is trivial so that $I_{0}(q)=\operatorname{Irr}(\Gamma)$.

(b) Let $\gamma=\left\{\gamma_{k}\right\}_{k}$ be a finite subset of $G^{\prime}$, where $\gamma_{k}=\left(\alpha_{k} \in G, y_{k} \in \Gamma\right)$ and $\left\{\alpha_{k}\right\}_{k}$ generate a free group $H \subset G$ of rank $|\gamma|$. Let

$$
\text { ә: } H_{2}(G, H ; \mathbb{Z}) \rightarrow H_{1}(H ; \mathbb{Z})=\bigoplus_{k} \mathbb{Z} \cdot\left[\alpha_{k}\right]
$$

be the boundary homomorphism, where $\left[\alpha_{k}\right] \in H_{1}(H ; \mathbb{Z})$ is the class of $\alpha_{k}$. For any $\rho \in \operatorname{Irr}(\Gamma)$ and any $Z \in H_{2}(G, H ; \mathbb{Z})$ such that $\partial Z=\sum_{k}\left[\alpha_{k}\right]$,

$$
\zeta^{\rho, \gamma}(Z) \prod_{k} t_{\rho}\left(\gamma_{k}\right)=\prod_{k} \operatorname{Tr} \rho\left(y_{k}\right) .
$$


Proof. Claim (a) is obvious. We prove (b). We first compute the function $t_{\rho}: G^{\prime} \rightarrow$ $\{0,1\}$. Let $a=(\alpha, y) \in G^{\prime}$ with $\alpha \in G$ and $y \in \Gamma$. Then $a \rho=y \rho=M^{-1} \rho M$ for $M=\rho(y)$. Thus, $t_{\rho}(a)=0$ if and only if $\operatorname{Tr} \rho(y)=0$. To prove (34) it suffices to consider the case where $\operatorname{Tr} \rho\left(y_{k}\right) \neq 0$ and $t_{\rho}\left(\gamma_{k}\right)=1$ for all $k$. Set $n=\operatorname{dim} \rho$. Define a lift $\alpha \mapsto \tilde{\alpha}^{\prime}$ of elements of $G$ to $G^{\prime}$ by $\tilde{\alpha}^{\prime}=(\alpha, 1)$ for $\alpha \in G-H$ and $\tilde{\alpha}^{\prime}=\left(\alpha, q^{-}(\alpha)\right)$ for $\alpha \in H$, where $q^{-}: H \rightarrow \Gamma$ is the homomorphism sending $\alpha_{k}$ to $y_{k}$ for all $k$. In the role of associated conjugating matrices (as in Section 2.2) we take $M_{\alpha}^{\prime}=E_{n}$ for $\alpha \in G-H$ and $M_{\alpha}^{\prime}=\rho\left(q^{-}(\alpha)\right)$ for $\alpha \in H$. It follows from the definitions that the corresponding 2-cocycle $\left\{\zeta_{\alpha, \beta}^{\prime}\right\}$ is trivial. The present choice of conjugating matrices $M_{\alpha}^{\prime}$ differs from the one in the definition of $\zeta^{\rho, \gamma}$ where it is arbitrary on $G-H$ and is given on $H$ by the homomorphism $\mu: H \rightarrow \operatorname{GL}_{n}(K)$ sending $\alpha_{k}$ to $\left(\operatorname{Tr} \rho\left(y_{k}\right)\right)^{-1} \rho\left(y_{k}\right)$ for all $k$. The homomorphisms $\mu, \rho q^{-}: H \rightarrow$ $\mathrm{GL}_{n}(K)$ are projectively equal, so there is a homomorphism $\psi: H \rightarrow K^{*}$ such that $\rho q^{-}=\psi \mu$. We extend $\psi$ to $G$ by $\psi(G-H)=1$ and conclude that $\zeta^{\rho, \gamma}$ is represented by the cocycle $\left\{\psi_{\alpha \beta}^{-1} \psi_{\alpha} \psi_{\beta}\right\}_{\alpha, \beta \in G}$. Clearly, $\psi\left(\alpha_{k}\right)=\operatorname{Tr} \rho\left(y_{k}\right)$ for all $k$. Therefore if $Z \in H_{2}(G, H ; \mathbb{Z})$ and $\partial Z=\sum_{k} r_{k}\left[\alpha_{k}\right]$ with $r_{k} \in \mathbb{Z}$, then $\zeta^{\rho, \gamma}(Z)=\prod_{k}\left(\operatorname{Tr} \rho\left(y_{k}\right)\right)^{r_{k}}$. This implies (34).

This lemma allows us to simplify formula (16) in the case where $G=\pi, g=$ id: $\pi \rightarrow \pi, G^{\prime}=G \times \Gamma$, and $q: G^{\prime} \rightarrow G$ is the projection. This gives the following theorem. Let $W$ be a compact connected oriented surface of positive genus $d$ with $m \geq 0$ boundary components and base point $w$. Let $x_{1}, \ldots, x_{m} \in \pi_{1}(W, w)$ be as in Section 5.1. Then for any finite group $\Gamma$ and any conjugacy classes $L_{1}, \ldots, L_{m}$ in $\Gamma$, the number of homomorphisms $\pi_{1}(W, w) \rightarrow \Gamma$ sending $x_{k}$ to $L_{k}$ for all $k=1, \ldots, m$ is equal to

$$
|\Gamma|^{2 d-1} \sum_{\rho \in \operatorname{Irr}(\Gamma)}\left\{(\operatorname{dim} \rho)^{2-2 d-m} \prod_{k=1}^{m} \sum_{y \in L_{k}} \operatorname{Tr} \rho(y)\right\} .
$$

This theorem is due to Frobenius [6] for $W=S^{1} \times S^{1}$ and to Mednykh [9] for all $W$; see also [8]. Theorem 1.2 for trivial bundles (over surfaces of positive genus) is an equivalent reformulation of the Frobenius-Mednykh theorem.

\section{References}

[1] C. Bachas and P. M. S. Petropoulos, Topological models on the lattice and a remark on string theory cloning. Comm. Math. Phys. 152 (1993), 191-202. MR 1207675 Zbl 0781.57005

[2] M. Brightwell and P. Turner, Representations of the homotopy surface category of a simply connected space. J. Knot Theory Ramifications 9 (2000), 855-864. MR 1780591 Zbl 0999.57030

[3] M. J. Collins, Representations and characters of finite groups. Cambridge Stud. Adv. Math. 22, Cambridge University Press, Cambridge 1990. MR 1050762 Zbl 0703.20001 
[4] R. Dijkgraaf and E. Witten, Topological gauge theories and group cohomology. Comm. Math. Phys. 129 (1990), 393-429. MR 1048699 Zbl 0703.58011

[5] D. S. Freed and F. Quinn, Chern-Simons theory with finite gauge group. Comm. Math. Phys. 156 (1993), 435-472. MR 1240583 Zbl 0788.58013

[6] G. Frobenius, Über Gruppencharaktere. Sitzber. Königlich Preuss. Akad. Wiss. Berlin 1896 (1896), 985-1021. JFM 27.0092.01

[7] M. Fukuma, S. Hosono, and H. Kawai, Lattice topological field theory in two dimensions. Comm. Math. Phys. 161 (1994), 157-175. MR 1266073 Zbl 0797.57012

[8] G. A. Jones, Enumeration of homomorphisms and surface-coverings. Quart. J. Math. Oxford Ser. (2) 46 (1995), 485-507. MR 1366619 Zbl 0859.57001

[9] A. D. Mednyh, Determination of the number of nonequivalent coverings over a compact Riemann surface. Dokl. Akad. Nauk SSSR 239 (1978), 269-271; English transl. Soviet Math. Dokl. 19 (1978), 318-320. MR 490616 Zbl 0395.30034

[10] M. Natapov and V. Turaev, On certain enumeration problems in group theory. Preprint 2009. arXiv:0904.3902

[11] V. G. Turaev, Quantum invariants of knots and 3-manifolds. de Gruyter Stud. Math. 18, Walter de Gruyter \& Co., Berlin 1994. MR 1292673 Zbl 0812.57003

[12] V. Turaev, Homotopy field theory in dimension 2 and group-algebras. Preprint 1999. arXiv:math/9910010

[13] V. Turaev, On certain enumeration problems in two-dimensional topology. Math. Res. Lett. 16 (2009), 515-529. MR 2511630 Zbl 1175.57013

[14] P. Turner, A functorial approach to differential characters. Algebr. Geom. Topol. 4 (2004), 81-93. MR 2031913 Zbl 1058.57024

Received November 10, 2009

V. Turaev, Department of Mathematics, Indiana University, Bloomington, IN 47405, U.S.A.

E-mail: vtouraev@indiana.edu 